\title{
Ancillary Tethering Influences $\sigma^{3}$-P vs. $\sigma^{5}$-P Speciation and Enables Intermolecular S-H Oxidative Addition to Nontrigonal Phosphorus Compounds
}

\author{
Hye Won Moon, ${ }^{\dagger}$ Ayan Maity, ${ }^{\dagger}$ and Alexander T. Radosevich* \\ Department of Chemistry, Massachusetts Institute of Technology, Cambridge, Massachusetts 02139, United States \\ ${ }^{\dagger}$ Authors contributed equally. \\ Correspondence to: radosevich@mit.edu
}

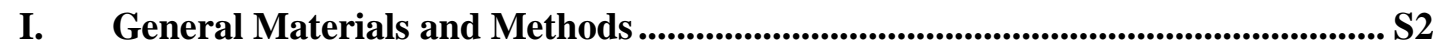

II. Preparation of Phosphorus Compounds..................................................................

III. Variable Temperature Experiments of Compound 2 ......................................... S5

IV. Oxidative Addition of E-H to Compound 1 and 2 ................................................S7

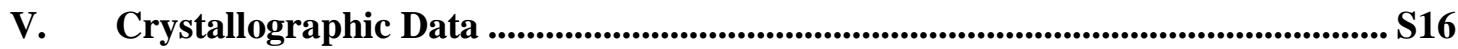

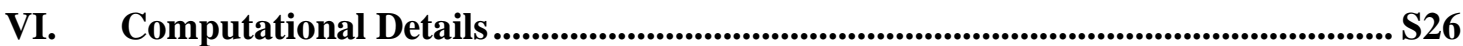

VII. Multinuclear NMR Spectra ..................................................................................... S29

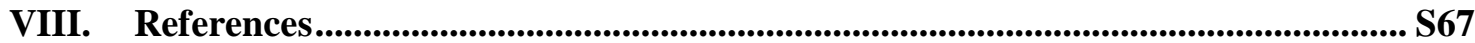




\section{General Materials and Methods}

All reactions were carried out using dry glassware and standard Schlenk techniques (when applicable) unless otherwise noted. All reagents were purchased from commercial vendors (Sigma-Aldrich, Alfa Aesar, Acros, TCI, or Oakwood Chemical) and used as received unless otherwise noted. Compound 1 was prepared according to literature procedures. ${ }^{1}$ All solvents were purified and collected under argon using a Glass Contour Solvent Purification System. ${ }^{1} \mathrm{H},{ }^{13} \mathrm{C}$, and ${ }^{31} \mathrm{P}$ NMR spectra were recorded with either Bruker AVANCE-400, or JEOL ECZR-500 spectrometers and processed using MestReNova. ${ }^{1} \mathrm{H}$ NMR chemical shifts are given in ppm with respect to the residual $\mathrm{CDCl}_{3}$ peak $(\delta 7.26 \mathrm{ppm})$, residual $\mathrm{CD}_{3} \mathrm{OD}(\delta$ $4.87 \mathrm{ppm})$, residual toluene- $d_{8}(\delta 2.09 \mathrm{ppm}),{ }^{13} \mathrm{C}\left\{{ }^{1} \mathrm{H}\right\}$ NMR shifts are given in ppm with respect to $\mathrm{CDCl}_{3}$ $(\delta 77.16 \mathrm{ppm})$, residual $\mathrm{CD}_{3} \mathrm{OD}(\delta 49.00 \mathrm{ppm})$, residual toluene- $d_{8}(\delta 20.40 \mathrm{ppm})$, and ${ }^{31} \mathrm{P}$ shifts are given in ppm with respect to $85 \% \mathrm{H}_{3} \mathrm{PO}_{4}(\delta 0.0 \mathrm{ppm})$. Coupling constants are reported as $J$-values in $\mathrm{Hz}$. High resolution DART mass spectra were obtained with JEOL AccuTOF DART. 


\section{Preparation of Phosphorus Compounds.}

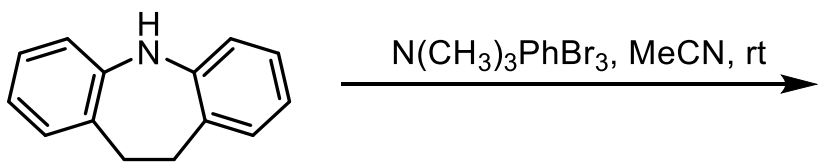

3

isoamyl nitrite, $\mathrm{HNO}_{3} / \mathrm{HOAC}, 0^{\circ} \mathrm{C}$

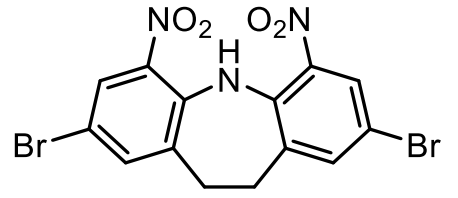

4

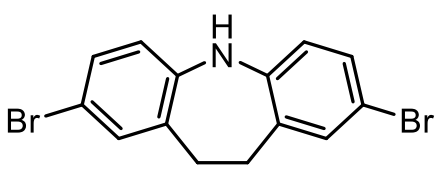

S1
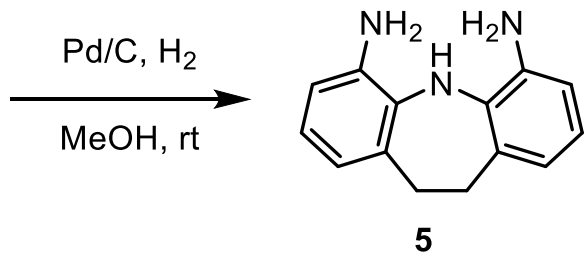

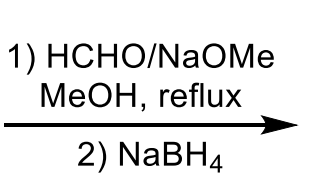<smiles>CNc1cccc2c1Nc1c(cccc1NC)CC2</smiles>

6

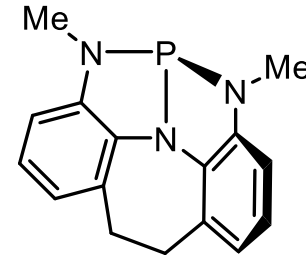

2

2,8-dibromo-10,11-dihydro-5H-dibenz[b,f]azepine (4): This procedure was adapted from previously published reports. ${ }^{2}$ 2,2'-iminodibenzyl $3(10 \mathrm{~g}, 0.0512 \mathrm{~mol})$, trimethylphenylammonium tribromide (38.5 $\mathrm{g}, 0.1024 \mathrm{~mol}$ ), and $300 \mathrm{~mL}$ dry acetonitrile were mixed under nitrogen atmosphere. After $4 \mathrm{~h}, 200 \mathrm{~mL}$ of water was added into the reaction mixture and the precipitate was filtered and dried under vacuum to afford 2,8-dibromoiminobenzyl (17.3 g, 96\% yield). Spectral data are in agreement with the literature reported values.

2,8-dibromo-4,6-dinitro-10,11-dihydro-5H-dibenzo[b,f]azepine (S1): This procedure was adapted from previously published reports. ${ }^{3} \mathrm{~A}$ round-bottom flask equipped with a stir bar was charged with a mixture of concentrated nitric acid $(70 \% ; 40 \mathrm{~mL})$ and glacial acetic acid $(180 \mathrm{~mL})$. The mixture was cooled to $0{ }^{\circ} \mathrm{C}$, 4 (17.3 g, $0.049 \mathrm{~mol})$ was added. Isoamyl nitrite $(20 \mathrm{~mL}, 0.147 \mathrm{~mol}, 3$ equiv) was slowly added to the mixture. The solution turned to red and was stirred for $30 \mathrm{~min}$ at $0{ }^{\circ} \mathrm{C}$. Red precipitates were filtered and washed with water $(500 \mathrm{~mL})$ and methanol $(100 \mathrm{~mL})$. The product was collected as a red solid and dried under vacuum (18.3 g, 84\% yield). This was used for next step without further purification. ${ }^{1} \mathrm{H}$ NMR $\left(\mathrm{CDCl}_{3}, 500 \mathrm{MHz}\right) \delta 12.02(\mathrm{~s}, 1 \mathrm{H}), 8.20(\mathrm{~d}, J=2.3 \mathrm{~Hz}, 2 \mathrm{H}), 7.46(\mathrm{~d}, J=2.3 \mathrm{~Hz}, 2 \mathrm{H}), 3.16(\mathrm{~s}, 4 \mathrm{H}) \mathrm{ppm}$. HRMS (DART) calculated for $\mathrm{C}_{14} \mathrm{H}_{10} \mathrm{Br}_{2} \mathrm{~N}_{3} \mathrm{O}_{4}(\mathrm{M}+\mathrm{H}) 441.9033$ found 441.9037.

10,11-dihydro-5H-dibenz[b,f]azepine-4,6-diamine (5): $\mathbf{S 1}(2 \mathrm{~g}, 9.028 \mathrm{mmol})$ and $\mathrm{Pd} / \mathrm{C}(150 \mathrm{mg})$ were mixed in $70 \mathrm{~mL}$ of methanol. The reaction mixture was transferred into a high-pressure reactor, charged with hydrogen (400 psi). After 12h, volatiles were removed without exposure to air. The resulting solids were brought into glovebox, triturated with $100 \mathrm{~mL}$ of methanol, and filtered through celite. The filtrate was concentrated and washed with $2 \mathrm{~mL}$ of methanol to give 5 as a white solid (677 $\mathrm{mg}, 66 \%$ yield). ${ }^{1} \mathrm{H}$ 
NMR $\left(\mathrm{CD}_{3} \mathrm{OD}, 400 \mathrm{MHz}\right) \delta 7.242(\mathrm{t}, J=7.5 \mathrm{~Hz}, 2 \mathrm{H}), 7.237(\mathrm{t}, J=7.5 \mathrm{~Hz}, 2 \mathrm{H}), 7.05(\mathrm{t}, J=7.5 \mathrm{~Hz}, 2 \mathrm{H})$, $3.17(\mathrm{~s}, 4 \mathrm{H}) \mathrm{ppm} .{ }^{13} \mathrm{C} \mathrm{NMR}\left(\mathrm{CD}_{3} \mathrm{OD}, 101 \mathrm{MHz}\right) \delta 137.14,136.94,132.25,123.99,123.85,123.24,35.45$ ppm. HRMS (DART) calculated for $\mathrm{C}_{14} \mathrm{H}_{16} \mathrm{~N}_{3}(\mathrm{M}+\mathrm{H}) 226.1339$ found 226.1337.

$N^{4}, N^{6}$-dimethyl-10,11-dihydro-5H-dibenz[b,f]azepine-4,6-diamine (6): Synthesized according to a modified literature procedure. ${ }^{1}$ A $500 \mathrm{~mL}$ Schlenk flask was charged with 5 (950 mg, $4.2 \mathrm{mmol}$ ) and paraformaldehyde $(1.27 \mathrm{~g}, 42 \mathrm{mmol}, 10$ equiv) in methanol $(40 \mathrm{~mL})$ in glovebox. To this mixture was slowly added a solution of $\mathrm{NaOMe}\left(911 \mathrm{mg}, 16.9 \mathrm{mmol}, 4\right.$ equiv) in $\mathrm{MeOH}(12 \mathrm{~mL})$ at $0{ }^{\circ} \mathrm{C}$. The reaction mixture was refluxed for $1 \mathrm{~h}$. After being cooled to $0{ }^{\circ} \mathrm{C}, \mathrm{NaBH}_{4}(1.62 \mathrm{~g}, 42 \mathrm{mmol}, 10$ equiv) was added at once. The solution was again stirred with heating to reflux for $1 \mathrm{~h}$. The volatiles were removed and the Schlenk flask was brought into glovebox. The resulting solids were redissolved in DCM $(60 \mathrm{~mL})$ and the solution was filtered through glass frit. The volatiles were removed under vacuum. The resulting solid was washed with pentane and dried under vacuum to afford the product as an off-white solid (310 $\mathrm{mg}, 32 \%)$. ${ }^{1} \mathrm{H} \mathrm{NMR}\left(\mathrm{CDCl}_{3}, 400 \mathrm{MHz}\right) \delta 6.80(\mathrm{~s}, 1 \mathrm{H}), 6.78-6.63(\mathrm{~m}, 6 \mathrm{H}), 3.10(\mathrm{~s}, 4 \mathrm{H}), 3.08(\mathrm{~d}, J=5.3 \mathrm{~Hz}, 2 \mathrm{H}), 2.89$ $(\mathrm{d}, J=5.3 \mathrm{~Hz}, 6 \mathrm{H}) \mathrm{ppm} .{ }^{13} \mathrm{C} \mathrm{NMR}\left(\mathrm{CDCl}_{3}, 101 \mathrm{MHz}\right) \delta 138.20,133.47,129.91,122.74,119.34,112.80$, 35.39, 32.72 ppm. HRMS (DART) calculated for $\mathrm{C}_{16} \mathrm{H}_{20} \mathrm{~N}_{3}(\mathrm{M}+\mathrm{H}) 254.1652$ found 254.1662.

Phosphorus (III) 2: Synthesized according to a modified literature procedure. ${ }^{1}$ To a solution of $\mathrm{PCl}_{3}(0.44$ $\mathrm{mL}, 5.0 \mathrm{mmol})$ in THF $(15 \mathrm{~mL})$ under $\mathrm{N}_{2}$ at $-78^{\circ} \mathrm{C}$ was added triamine ligand $(1.41 \mathrm{~g}, 5.0 \mathrm{mmol})$ in ether $(25 \mathrm{~mL})$ dropwise via syringe. Triethylamine $(2.1 \mathrm{~mL}, 15.0 \mathrm{mmol})$ in ether $(10 \mathrm{~mL})$ was then added dropwise, and the resulting mixture was warmed to room temperature and stirred for an additional $4 \mathrm{~h}$ giving a white suspension within a light-yellow solution. The volatiles were removed in vacuo. The resulting solids were brought into glovebox, redissolved in ether and benzene, and filtered through celite. The filtrate was again concentrated to give yellow solids, which was further recrystallized from a mixture of ether and DCM at $-35{ }^{\circ} \mathrm{C}$ to give 2 as white crystals (1.06 g, $67 \%$ yield). ${ }^{1} \mathrm{H} \mathrm{NMR}\left(\mathrm{CDCl}_{3}, 400 \mathrm{MHz}\right)$ $\delta 6.87(\mathrm{t}, J=7.7 \mathrm{~Hz}, 2 \mathrm{H}), 6.63(\mathrm{~d}, J=7.7 \mathrm{~Hz}, 2 \mathrm{H}), 6.45(\mathrm{~d}, J=7.7 \mathrm{~Hz}, 2 \mathrm{H}), 3.22(\mathrm{~s}, 4 \mathrm{H}), 3.07\left(\mathrm{~d},{ }^{3} J_{\mathrm{P}-\mathrm{H}}=\right.$ $8.1 \mathrm{~Hz}, 6 \mathrm{H}) \mathrm{ppm} .{ }^{13} \mathrm{C} \mathrm{NMR}\left(\mathrm{CDCl}_{3}, 101 \mathrm{MHz}\right) \delta 140.16\left(\mathrm{~d},{ }^{2} J_{\mathrm{P}-\mathrm{C}}=8.0 \mathrm{~Hz}\right), 138.50,128.93\left(\mathrm{~d},{ }^{2} J_{\mathrm{P}-\mathrm{C}}=5.6\right.$ $\mathrm{Hz}), 123.21,120.11,106.37,29.94,29.63\left(\mathrm{~d},{ }^{2} J_{\mathrm{P}-\mathrm{C}}=31.5 \mathrm{~Hz}\right) \mathrm{ppm} .{ }^{31} \mathrm{P} \mathrm{NMR}\left(\mathrm{CDCl}_{3}, 162 \mathrm{MHz}\right) \delta 154.21$ ppm. HRMS (DART) calculated for $\mathrm{C}_{16} \mathrm{H}_{17} \mathrm{~N}_{3} \mathrm{P}(\mathrm{M}+\mathrm{H}) 282.1155$ found 282.1158 .

Phosphorus selenium adduct 2•[Se]: Phosphorus(III) compound 2 (15 mg, $0.05 \mathrm{mmol})$ was dissolved in $\mathrm{CDCl}_{3}(1 \mathrm{~mL})$. Elemental selenium powder $(4.8 \mathrm{mg}, 0.06 \mathrm{mmol})$ was then added. The reaction was stirred at $50{ }^{\circ} \mathrm{C}$ for $2 \mathrm{~h}$ to give quantitative conversion. Excess selenium powder was removed via filtration over Celite. ${ }^{1} \mathrm{H} \mathrm{NMR}\left(\mathrm{CDCl}_{3}, 400 \mathrm{MHz}\right) \delta 6.95(\mathrm{t}, J=7.8 \mathrm{~Hz}, 2 \mathrm{H}), 6.71(\mathrm{~d}, J=7.8 \mathrm{~Hz}, 2 \mathrm{H}), 6.51(\mathrm{~d}, J=7.8 \mathrm{~Hz}$, $2 \mathrm{H}), 3.57-3.46(\mathrm{~m}, 2 \mathrm{H}), 3.16(\mathrm{~d}, J=12.2 \mathrm{~Hz}, 6 \mathrm{H}), 2.97-2.85(\mathrm{~m}, 1 \mathrm{H}) \mathrm{ppm} .{ }^{13} \mathrm{C} \mathrm{NMR}\left(\mathrm{CDCl}_{3}, 101 \mathrm{MHz}\right)$ $\delta 137.40\left(\mathrm{~d},{ }^{2} J_{\mathrm{P}-\mathrm{C}}=14.4 \mathrm{~Hz}\right), 134.35\left(\mathrm{~d},{ }^{3} J_{\mathrm{P}-\mathrm{C}}=8.6 \mathrm{~Hz}\right), 129.19\left(\mathrm{~d},{ }^{2} J_{\mathrm{P}-\mathrm{C}}=10.3 \mathrm{~Hz}\right), 124.18,121.70$, $106.77\left(\mathrm{~d},{ }^{3} J_{\mathrm{P}-\mathrm{C}}=8.4 \mathrm{~Hz}\right), 30.09\left(\mathrm{~d},{ }^{2} J_{\mathrm{P}-\mathrm{C}}=7.3 \mathrm{~Hz}\right), 29.55 \mathrm{ppm} .{ }^{31} \mathrm{P} \mathrm{NMR}\left(\mathrm{CDCl}_{3}, 162 \mathrm{MHz}\right) \delta 89.08$, $89.08\left(\mathrm{~d},{ }^{1} J_{\mathrm{P}-\mathrm{Se}}=906.2 \mathrm{~Hz}\right) \mathrm{ppm}$. HRMS $(\mathrm{DART})$ calculated for $\mathrm{C}_{16} \mathrm{H}_{17} \mathrm{~N}_{3} \mathrm{PSe}(\mathrm{M}+\mathrm{H}) 362.0320$ found 362.0303 . 


\section{Variable Temperature Experiments of Compound 2}

Experimental ${ }^{1} H$ NMR of compound 2: In an inert atmosphere glovebox, compound 2 (30 $\mathrm{mg}$ ) was dissolved in toluene- $d_{8}(1 \mathrm{~mL})$. The sample was then transferred to a sealable J-Young NMR tube. Variable temperature NMR experiments were recorded on a Bruker DPX-400 spectrometer. ${ }^{1} \mathrm{H}$ and ${ }^{31} \mathrm{P}$ NMR spectra were recorded at temperature intervals between $-10{ }^{\circ} \mathrm{C}>\mathrm{T}>-90{ }^{\circ} \mathrm{C}$. The sample and probe were thermostatted at each temperature for 5 minutes before spectra were recorded. At the end of the experiment, a spectrum at room temperature was recorded to verify the reversibility of the temperature dependent process.

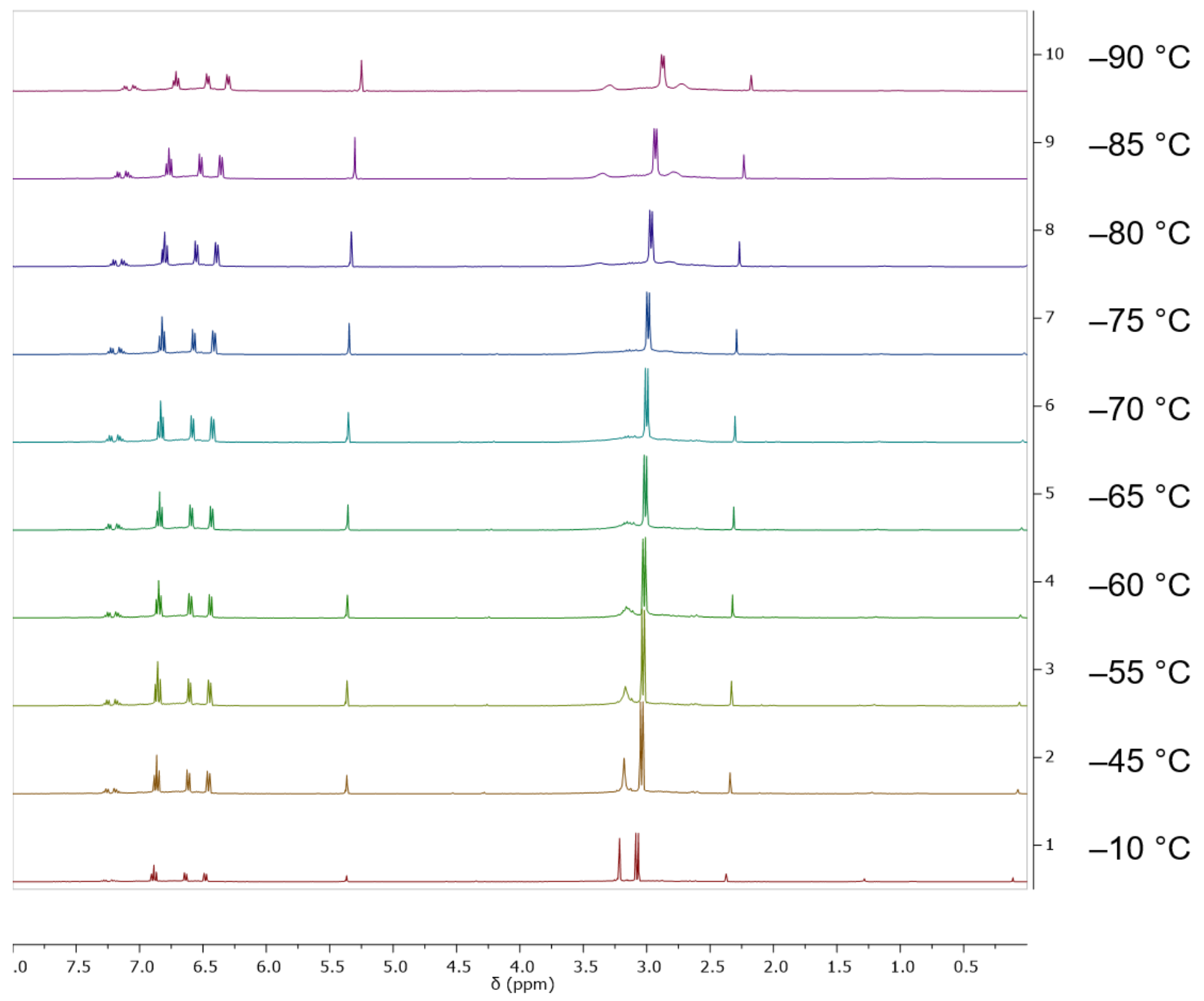

Figure S1. Stacked ${ }^{1} \mathrm{H}$ NMR spectra of compound 2 at temperatures in the range $-10{ }^{\circ} \mathrm{C}>\mathrm{T}>-90{ }^{\circ} \mathrm{C}$. 
Simulation of ${ }^{1} H$ NMR of compound 2: Line-shape fitting was performed using the WinDNMR software package. ${ }^{4}$ All chemical shift, coupling, and line width values were set based on values derived from the experimental spectra. The exchange rate variable was adjusted to fit the overlaid experimental spectrum at each temperature.

Table S1. Simulated rate constants for edge inversion of 2.

\begin{tabular}{cccc}
\hline $\boldsymbol{k}\left(\mathbf{s}^{-\mathbf{1}}\right)$ & $\mathbf{T}(\mathbf{K})$ & $\mathbf{1} / \mathbf{T}\left(\mathbf{K}^{-\mathbf{1}}\right)$ & $\mathbf{l n}(\boldsymbol{k} / \mathbf{T})$ \\
\hline 25.6 & 183 & 0.00546 & -1.96777 \\
264 & 188 & 0.005315 & 0.338656 \\
310 & 193 & 0.005177 & 0.473053 \\
940 & 198 & 0.005046 & 1.556805 \\
1191 & 203 & 0.004922 & 1.768555 \\
1331 & 208 & 0.004804 & 1.855379 \\
2961 & 213 & 0.004691 & 2.631239 \\
10031 & 218 & 0.004584 & 3.828207 \\
19672 & 228 & 0.004383 & 4.456904 \\
162000 & 263 & 0.0038 & 6.422589 \\
\hline
\end{tabular}

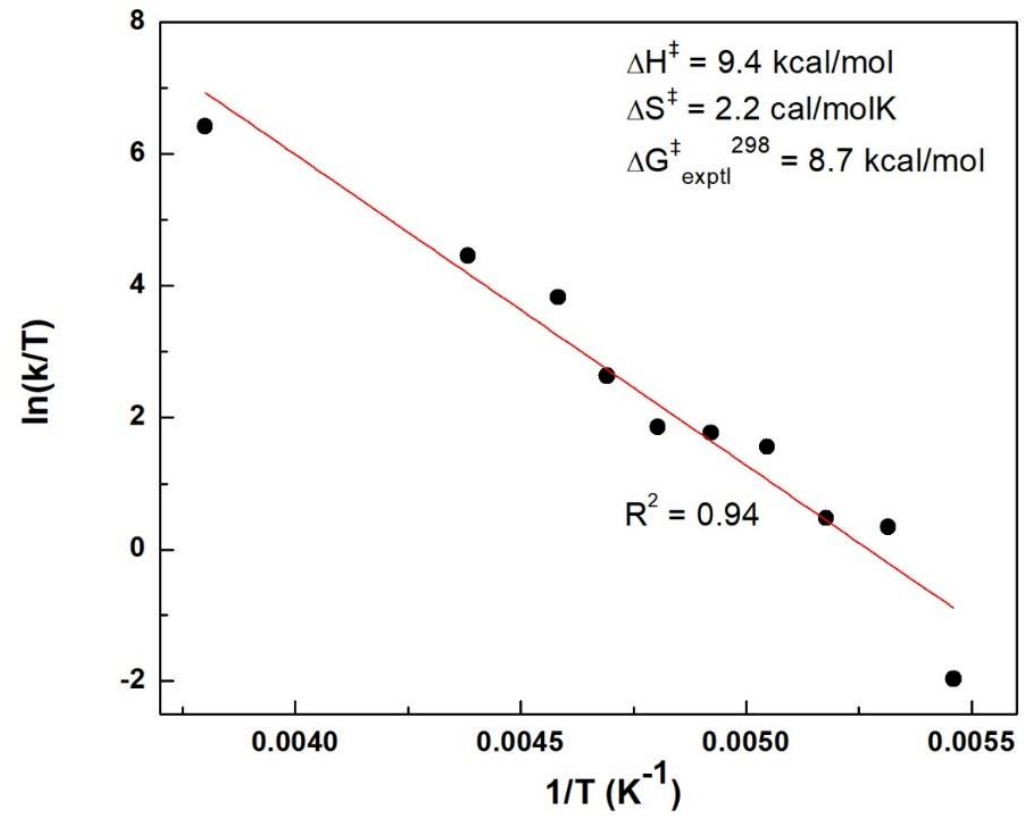

Figure S2. Eyring plot for the edge inversion of $\mathbf{2}$ derived from simulated rate constants. 


\section{Oxidative Addition of E-H to Compound 1 and 2}

General procedure for oxidative $O-H$ additions to 1 : To a solution of phosphorus(III) compound 1 (12.8 $\mathrm{mg}, 0.05 \mathrm{mmol}, 1$ equiv) in dry $\mathrm{CDCl}_{3}(0.5 \mathrm{~mL})$ was added a solution of $\mathrm{ROH}(0.05 \mathrm{mmol}, 1$ equiv) in dry $\mathrm{CDCl}_{3}(0.5 \mathrm{~mL})$. The solution was then stirred and was monitored by ${ }^{31} \mathrm{P}$ NMR until complete conversion to corresponding phosphorus(V) species was observed or the reaction reached an equilibrium of tautomerism (typically ca. $24 \mathrm{~h}$ ).

General procedure for oxidative $\mathrm{O}-\mathrm{H}$ additions to 2 : To a solution of phosphorus(III) compound 2 (14 $\mathrm{mg}$, 0.05 mmol, 1 equiv) in dry $\mathrm{CDCl}_{3}(0.5 \mathrm{~mL})$ was added a solution of $\mathrm{ROH}(0.05 \mathrm{mmol}, 1$ equiv) in dry $\mathrm{CDCl}_{3}(0.5 \mathrm{~mL})$. The solution was then stirred and was monitored by ${ }^{31} \mathrm{P}$ NMR until complete conversion to corresponding phosphorus(V) species was observed (typically in $15 \mathrm{~min}$ ).

Procedure for oxidative $S-H$ additions to 2: To a solution of phosphorus(III) compound 2 (14 mg, 0.05 mmol, 1 equiv) in dry toluene- $d_{8}(0.5 \mathrm{~mL})$ was added a solution of $\mathrm{RSH}\left(0.1 \mathrm{mmol}, 2\right.$ equiv) in dry $\mathrm{CDCl}_{3}$ $(0.5 \mathrm{~mL})$. The solution was then stirred and was monitored by ${ }^{31} \mathrm{P}$ NMR until complete conversion to corresponding phosphorus(V) species was observed (in $48 \mathrm{hr}$ ).

\section{$2 \cdot[\mathrm{H}][\mathrm{O} t-\mathrm{Bu}]$}

${ }^{1} \mathrm{H} \mathrm{NMR}\left(400 \mathrm{MHz}, \mathrm{CDCl}_{3}\right) \delta 6.84(\mathrm{t}, J=7.6 \mathrm{~Hz}, 2 \mathrm{H}), 6.69(\mathrm{~d}, J=7.7 \mathrm{~Hz}, 2 \mathrm{H}), 6.63(\mathrm{~d}, J=7.6 \mathrm{~Hz}, 2 \mathrm{H})$, $6.41\left(\mathrm{~d},{ }^{1} J_{\mathrm{P}-\mathrm{H}}=597.5 \mathrm{~Hz}, 1 \mathrm{H}\right), 3.37-3.30(\mathrm{~m}, 2 \mathrm{H}), 3.13-3.04(\mathrm{~m}, 2 \mathrm{H}), 3.06\left(\mathrm{~d},{ }^{3} J_{\mathrm{P}-\mathrm{H}}=17.2 \mathrm{~Hz}, 6 \mathrm{H}\right), 1.11$ (s, 16H) ppm. ${ }^{13} \mathrm{C} \mathrm{NMR}\left(101 \mathrm{MHz}, \mathrm{CDCl}_{3}\right) \delta 132.55\left(\mathrm{~d},{ }^{2} J_{\mathrm{P}-\mathrm{C}}=12.1 \mathrm{~Hz}\right), 130.11\left(\mathrm{~d},{ }^{2} J_{\mathrm{P}-\mathrm{C}}=15.9 \mathrm{~Hz}\right)$, $125.19\left(\mathrm{~d},{ }^{3} J_{\mathrm{P}-\mathrm{C}}=10.6 \mathrm{~Hz}\right), 120.13\left(\mathrm{~d},{ }^{4} J_{\mathrm{P}-\mathrm{C}}=2.1 \mathrm{~Hz}\right), 119.19,106.45\left(\mathrm{~d},{ }^{3} J_{\mathrm{P}-\mathrm{C}}=7.1 \mathrm{~Hz}\right), 75.00\left(\mathrm{~d},{ }^{2} J_{\mathrm{P}-\mathrm{C}}=\right.$ $8.7 \mathrm{~Hz}), 33.25,30.40\left(\mathrm{~d},{ }^{3} J_{\mathrm{P}-\mathrm{C}}=5.9 \mathrm{~Hz}\right), 28.60\left(\mathrm{~d},{ }^{2} J_{\mathrm{P}-\mathrm{C}}=18.2 \mathrm{~Hz}\right) \mathrm{ppm} .{ }^{31} \mathrm{P}\left\{{ }^{1} \mathrm{H}\right\} \mathrm{NMR}\left(162 \mathrm{MHz}, \mathrm{CDCl}_{3}\right)$ $\delta-37.62$ ppm. ${ }^{31} \mathrm{P}$ NMR (162 MHz, $\left.\mathrm{CDCl}_{3}\right) \delta-37.62$ (dhept, $J=597.5,17.2 \mathrm{~Hz}$ ) ppm. HRMS (DART) calculated for $\mathrm{C}_{20} \mathrm{H}_{27} \mathrm{~N}_{3} \mathrm{OP}(\mathrm{M}+\mathrm{H}) 356.1886$ found 356.1887 .

$2 \cdot[\mathrm{H}][\mathrm{O}-1-\mathrm{Ad}]$

${ }^{1} \mathrm{H}$ NMR $\left(400 \mathrm{MHz}, \mathrm{CDCl}_{3}\right) \delta 6.85(\mathrm{t}, J=7.6 \mathrm{~Hz}, 2 \mathrm{H}), 6.69(\mathrm{~d}, J=7.6 \mathrm{~Hz}, 2 \mathrm{H}), 6.63(\mathrm{~d}, J=7.6 \mathrm{~Hz}, 2 \mathrm{H})$, $6.48\left(\mathrm{~d},{ }^{1} J_{\mathrm{P}-\mathrm{H}}=599.5 \mathrm{~Hz}, 1 \mathrm{H}\right), 3.40-3.26(\mathrm{~m}, 2 \mathrm{H}), 3.15-3.04(\mathrm{~m}, 2 \mathrm{H}), 3.06\left(\mathrm{~d},{ }^{3} J_{\mathrm{P}-\mathrm{H}}=17.1 \mathrm{~Hz}, 6 \mathrm{H}\right), 2.02$ $(\mathrm{s}, 3 \mathrm{H}), 1.71-1.46(\mathrm{~m}, 12 \mathrm{H}) \mathrm{ppm} .{ }^{13} \mathrm{C} \mathrm{NMR}\left(101 \mathrm{MHz}, \mathrm{CDCl}_{3}\right) \delta 132.52\left(\mathrm{~d},{ }^{2} J_{\mathrm{P}-\mathrm{C}}=12.3 \mathrm{~Hz}\right), 129.96(\mathrm{~d}$, $\left.{ }^{2} J_{\mathrm{P}-\mathrm{C}}=16.0 \mathrm{~Hz}\right), 125.15\left(\mathrm{~d},{ }^{3} J_{\mathrm{P}-\mathrm{C}}=10.4 \mathrm{~Hz}\right), 120.06\left(\mathrm{~d},{ }^{4} J_{\mathrm{P}-\mathrm{C}}=1.9 \mathrm{~Hz}\right), 119.14,106.39\left(\mathrm{~d},{ }^{3} J_{\mathrm{P}-\mathrm{C}}=7.4 \mathrm{~Hz}\right)$, $74.87\left(\mathrm{~d},{ }^{2} J_{\mathrm{P}-\mathrm{C}}=9.4 \mathrm{~Hz}\right), 43.93\left(\mathrm{~d},{ }^{4} J_{\mathrm{P}-\mathrm{C}}=5.5 \mathrm{~Hz}\right), 36.22,33.32,30.99,28.52\left(\mathrm{~d},{ }^{2} J_{\mathrm{P}-\mathrm{C}}=18.3 \mathrm{~Hz}\right) \mathrm{ppm}$. ${ }^{31} \mathrm{P}\left\{{ }^{1} \mathrm{H}\right\} \mathrm{NMR}\left(162 \mathrm{MHz}, \mathrm{CDCl}_{3}\right) \delta-39.87 \mathrm{ppm} .{ }^{31} \mathrm{P} \mathrm{NMR}\left(162 \mathrm{MHz}, \mathrm{CDCl}_{3}\right) \delta-39.87(\mathrm{dhept}, J=599.5$, $17.1 \mathrm{~Hz})$ ppm. HRMS (DART) calculated for $\mathrm{C}_{26} \mathrm{H}_{33} \mathrm{~N}_{3} \mathrm{OP}(\mathrm{M}+\mathrm{H}) 434.2356$ found 434.2362.

\section{$2 \cdot[\mathrm{H}]\left[\mathrm{OCPh}_{3}\right]$}

${ }^{1} \mathrm{H}$ NMR $\left(400 \mathrm{MHz}, \mathrm{CDCl}_{3}\right) \delta 7.38-7.28(\mathrm{~m}, 6 \mathrm{H}), 7.23-7.14(\mathrm{~m}, 9 \mathrm{H}), 6.84(\mathrm{dd}, J=7.7,7.5 \mathrm{~Hz}, 2 \mathrm{H})$, $6.74(\mathrm{~d}, J=7.7 \mathrm{~Hz}, 2 \mathrm{H}), 6.50(\mathrm{~d}, J=7.5 \mathrm{~Hz}, 2 \mathrm{H}), 6.09\left(\mathrm{~d},{ }^{1} J_{\mathrm{P}-\mathrm{H}}=600.4 \mathrm{~Hz}, 1 \mathrm{H}\right), 3.68-2.84(\mathrm{~m}, 4 \mathrm{H}), 2.33$ $(\mathrm{d}, J=17.4 \mathrm{~Hz}, 6 \mathrm{H}) \mathrm{ppm} .{ }^{13} \mathrm{C} \mathrm{NMR}\left(101 \mathrm{MHz}, \mathrm{CDCl}_{3}\right) \delta 145.21\left(\mathrm{~d}, J_{\mathrm{P}-\mathrm{C}}=5.2 \mathrm{~Hz}\right), 131.83\left(\mathrm{~d},{ }^{2} J_{\mathrm{P}-\mathrm{C}}=13.0\right.$ 
$\mathrm{Hz}), 130.73\left(\mathrm{~d},{ }^{2} J_{\mathrm{P}-\mathrm{C}}=16.0 \mathrm{~Hz}\right), 128.65,127.81,127.04,125.16\left(\mathrm{~d}, J_{\mathrm{P}-\mathrm{C}}=10.9 \mathrm{~Hz}\right), 120.38\left(\mathrm{~d}, J_{\mathrm{P}-\mathrm{C}}=2.1\right.$ $\mathrm{Hz}), 119.21,106.73\left(\mathrm{~d}, J_{\mathrm{P}-\mathrm{C}}=7.4 \mathrm{~Hz}\right), 85.99\left(\mathrm{~d},{ }^{2} J_{\mathrm{P}-\mathrm{C}}=6.1 \mathrm{~Hz}\right), 33.07,28.13\left(\mathrm{~d},{ }^{2} J_{\mathrm{P}-\mathrm{C}}=18.0 \mathrm{~Hz}\right) \mathrm{ppm}$. ${ }^{31} \mathrm{P}\left\{{ }^{1} \mathrm{H}\right\} \mathrm{NMR}\left(162 \mathrm{MHz}, \mathrm{CDCl}_{3}\right) \delta-37.61 \mathrm{ppm} .{ }^{31} \mathrm{P} \mathrm{NMR}\left(162 \mathrm{MHz}, \mathrm{CDCl}_{3}\right) \delta-37.61$ (dhept, $J=600.4$, $17.4 \mathrm{~Hz}$ ) ppm. HRMS (DART) calculated for $\mathrm{C}_{35} \mathrm{H}_{33} \mathrm{~N}_{3} \mathrm{OP}(\mathrm{M}+\mathrm{H}) 542.2356$ found 542.2357.

$2 \cdot[\mathrm{H}]\left[\mathrm{O}-p-\mathrm{MeOC}_{6} \mathrm{H}_{4}\right]$

${ }^{1} \mathrm{H}$ NMR $\left(400 \mathrm{MHz}, \mathrm{CDCl}_{3}\right) \delta 6.88(\mathrm{t}, J=7.7 \mathrm{~Hz}, 2 \mathrm{H}), 6.79-6.61(\mathrm{~m}, 6 \mathrm{H}), 6.54(\mathrm{dd}, J=9.0,2.3 \mathrm{~Hz}, 2 \mathrm{H})$, $6.35\left(\mathrm{~d},{ }^{1} J_{\mathrm{P}-\mathrm{H}}=625.8 \mathrm{~Hz}, 1 \mathrm{H}\right), 3.72(\mathrm{~s}, 3 \mathrm{H}), 3.34-3.18(\mathrm{~m}, 2 \mathrm{H}), 3.12-2.97(\mathrm{~m}, 2 \mathrm{H}), 3.07\left(\mathrm{~d},{ }^{3} J_{\mathrm{P}-\mathrm{H}}=17.4\right.$ $\mathrm{Hz}, 6 \mathrm{H}) \mathrm{ppm} .{ }^{13} \mathrm{C} \mathrm{NMR}\left(101 \mathrm{MHz}, \mathrm{CDCl}_{3}\right) \delta 156.11(\mathrm{~d}, J=2.9 \mathrm{~Hz}), 145.73(\mathrm{~d}, J=8.6 \mathrm{~Hz}), 132.24(\mathrm{~d}, J=$ $11.5 \mathrm{~Hz}), 129.66(\mathrm{~d}, J=15.5 \mathrm{~Hz}), 125.54\left(\mathrm{~d},{ }^{3} J_{\mathrm{P}-\mathrm{C}}=10.5 \mathrm{~Hz}\right), 123.00\left(\mathrm{~d},{ }^{4} J_{\mathrm{P}-\mathrm{C}}=5.1 \mathrm{~Hz}\right), 120.72,119.67$, $114.29\left(\mathrm{~d},{ }^{4} J_{\mathrm{P}-\mathrm{C}}=2.9 \mathrm{~Hz}\right), 106.78\left(\mathrm{~d},{ }^{3} J_{\mathrm{P}-\mathrm{C}}=7.2 \mathrm{~Hz}\right), 55.66,33.21,28.52\left(\mathrm{~d},{ }^{2} J_{\mathrm{P}-\mathrm{C}}=17.4 \mathrm{~Hz}\right) \mathrm{ppm} .{ }^{31} \mathrm{P}\left\{{ }^{1} \mathrm{H}\right\}$ NMR (162 MHz, $\left.\mathrm{CDCl}_{3}\right) \delta-25.94 \mathrm{ppm} .{ }^{31} \mathrm{P} \mathrm{NMR}\left(162 \mathrm{MHz}, \mathrm{CDCl}_{3}\right) \delta-25.94$ (dhept $J=625.8,17.4 \mathrm{~Hz}$ ) ppm. HRMS (DART) calculated for $\mathrm{C}_{23} \mathrm{H}_{25} \mathrm{~N}_{3} \mathrm{O}_{2} \mathrm{P}(\mathrm{M}+\mathrm{H}) 406.1684$ found 406.1689 .

$2 \cdot[\mathrm{H}][\mathrm{OOC}-p-\mathrm{Tol}]$

${ }^{1} \mathrm{H}$ NMR $\left(400 \mathrm{MHz}, \mathrm{CDCl}_{3}\right) \delta 7.67(\mathrm{~d}, J=8.2 \mathrm{~Hz}, 2 \mathrm{H}), 7.15\left(\mathrm{~d},{ }^{1} J_{\mathrm{P}-\mathrm{H}}=657.5 \mathrm{~Hz}, 1 \mathrm{H}\right), 7.09(\mathrm{~d}, J=8.1 \mathrm{~Hz}$, $2 \mathrm{H}), 6.93(\mathrm{t}, J=7.7 \mathrm{~Hz}, 2 \mathrm{H}), 6.77(\mathrm{t}, J=7.5 \mathrm{~Hz}, 4 \mathrm{H}), 3.43-3.32(\mathrm{~m}, 2 \mathrm{H}), 3.23\left(\mathrm{~d},{ }^{3} J_{\mathrm{P}-\mathrm{H}}=18.8 \mathrm{~Hz}, 6 \mathrm{H}\right)$, $3.19-3.07(\mathrm{~m}, 2 \mathrm{H}), 2.33(\mathrm{~s}, 3 \mathrm{H}) \mathrm{ppm} .{ }^{13} \mathrm{C} \mathrm{NMR}\left(101 \mathrm{MHz}, \mathrm{CDCl}_{3}\right) \delta 163.49\left(\mathrm{~d},{ }^{2} J_{\mathrm{P}-\mathrm{C}}=6.4 \mathrm{~Hz}\right), 144.01$, $131.65\left(\mathrm{~d},{ }^{2} J_{\mathrm{P}-\mathrm{C}}=11.7 \mathrm{~Hz}\right), 130.28,129.08\left(\mathrm{~d},{ }^{2} J_{\mathrm{P}-\mathrm{C}}=17.2 \mathrm{~Hz}\right), 129.07,127.98\left(\mathrm{~d},{ }^{3} J_{\mathrm{P}-\mathrm{C}}=4.2 \mathrm{~Hz}\right), 125.50$ $\left(\mathrm{d},{ }^{3} J_{\mathrm{P}-\mathrm{C}}=11.5 \mathrm{~Hz}\right), 120.99\left(\mathrm{~d},{ }^{4} J_{\mathrm{P}-\mathrm{C}}=2.2 \mathrm{~Hz}\right), 119.89,107.29\left(\mathrm{~d},{ }^{3} J_{\mathrm{P}-\mathrm{C}}=7.4 \mathrm{~Hz}\right), 33.40,28.56\left(\mathrm{~d},{ }^{2} J_{\mathrm{P}-\mathrm{C}}=\right.$ $19.7 \mathrm{~Hz}), 21.77 \mathrm{ppm} .{ }^{31} \mathrm{P}\left\{{ }^{1} \mathrm{H}\right\} \mathrm{NMR}\left(162 \mathrm{MHz}, \mathrm{CDCl}_{3}\right) \delta-38.82 \mathrm{ppm} .{ }^{31} \mathrm{P} \mathrm{NMR}\left(162 \mathrm{MHz}, \mathrm{CDCl}_{3}\right) \delta-$ 38.82 (dhept, $J=657.5,18.8 \mathrm{~Hz}$ ) ppm. HRMS (DART) calculated for $\mathrm{C}_{24} \mathrm{H}_{25} \mathrm{~N}_{3} \mathrm{O}_{2} \mathrm{P}(\mathrm{M}+\mathrm{H}) 418.1679$ found 418.1680 .

$2 \cdot[\mathrm{H}][\mathrm{SBn}]$

${ }^{1} \mathrm{H}$ NMR $\left(400 \mathrm{MHz}\right.$, toluene- $\left.d_{8}\right) \delta 7.06-6.90(\mathrm{~m}, 5 \mathrm{H}), 6.86(\mathrm{t}, J=7.7 \mathrm{~Hz}, 2 \mathrm{H}), 6.67\left(\mathrm{~d},{ }^{1} J_{\mathrm{P}-\mathrm{H}}=563.5 \mathrm{~Hz}\right.$, $1 \mathrm{H}), 6.62(\mathrm{~d}, J=7.8 \mathrm{~Hz}, 2 \mathrm{H}), 6.42(\mathrm{~d}, J=7.6 \mathrm{~Hz}, 2 \mathrm{H}), 3.41\left(\mathrm{~d},{ }^{3} J_{\mathrm{P}-\mathrm{H}}=20.0 \mathrm{~Hz}, 2 \mathrm{H}\right), 3.11-3.02(\mathrm{~m}, 2 \mathrm{H})$, $2.85-2.75(\mathrm{~m}, 2 \mathrm{H}), 2.56\left(\mathrm{~d},{ }^{3} J_{\mathrm{P}-\mathrm{H}}=18.4 \mathrm{~Hz}, 6 \mathrm{H}\right) \mathrm{ppm} .{ }^{13} \mathrm{C}$ NMR $\left(101 \mathrm{MHz}\right.$, toluene- $\left.d_{8}\right) \delta 139.95\left(\mathrm{~d},{ }^{3} J_{\mathrm{P}-}\right.$ $\mathrm{C}=4.0 \mathrm{~Hz}), 132.69\left(\mathrm{~d},{ }^{2} J_{\mathrm{P}-\mathrm{C}}=12.3 \mathrm{~Hz}\right), 130.91\left(\mathrm{~d},{ }^{2} J_{\mathrm{P}-\mathrm{C}}=15.1 \mathrm{~Hz}\right), 128.80,128.39,126.70,125.54\left(\mathrm{~d},{ }^{3} J_{\mathrm{P}-}\right.$ $\mathrm{C}=9.7 \mathrm{~Hz}), 121.40\left(\mathrm{~d},{ }^{4} J_{\mathrm{P}-\mathrm{C}}=1.9 \mathrm{~Hz}\right), 120.69,107.58\left(\mathrm{~d},{ }^{3} J_{\mathrm{P}-\mathrm{C}}=7.3 \mathrm{~Hz}\right), 34.56,32.98,27.76\left(\mathrm{~d},{ }^{2} J_{\mathrm{P}-\mathrm{C}}=\right.$ $15.9 \mathrm{~Hz}$ ) ppm. ${ }^{31} \mathrm{P}\left\{{ }^{1} \mathrm{H}\right\}$ NMR (toluene- $d_{8}, 162 \mathrm{MHz}$ ): $-32.45 \mathrm{ppm} .{ }^{31} \mathrm{P}$ NMR (toluene- $d_{8}, 162 \mathrm{MHz}$ ): -32.45 (dthept, $J=563.5,20.0,18.4 \mathrm{~Hz}$ ) ppm. HRMS (DART) calculated for $\mathrm{C}_{23} \mathrm{H}_{25} \mathrm{~N}_{3} \mathrm{PS}(\mathrm{M}+\mathrm{H}) 406.1501$ found 406.1495 . 
A. O-H bond in tertiary alcohols.<smiles>CCO[C@@H]1N(C)c2ccccc2N1c1ccccc1N</smiles>

$1 \cdot[\mathrm{H}]\left[\mathrm{O}^{t} \mathrm{Bu}\right]$

$\delta-37.5 \mathrm{ppm}$

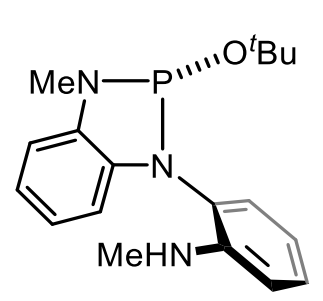

anti- $\sigma^{3}-\mathrm{P}$

$\delta 117.7 \mathrm{ppm}$

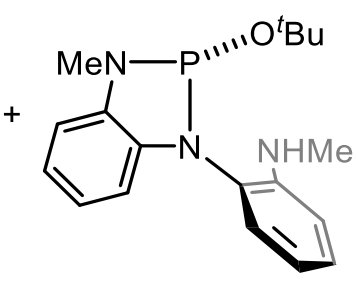

syn- $\sigma^{3}-\mathrm{P}$

$\delta 110.8$ ppm

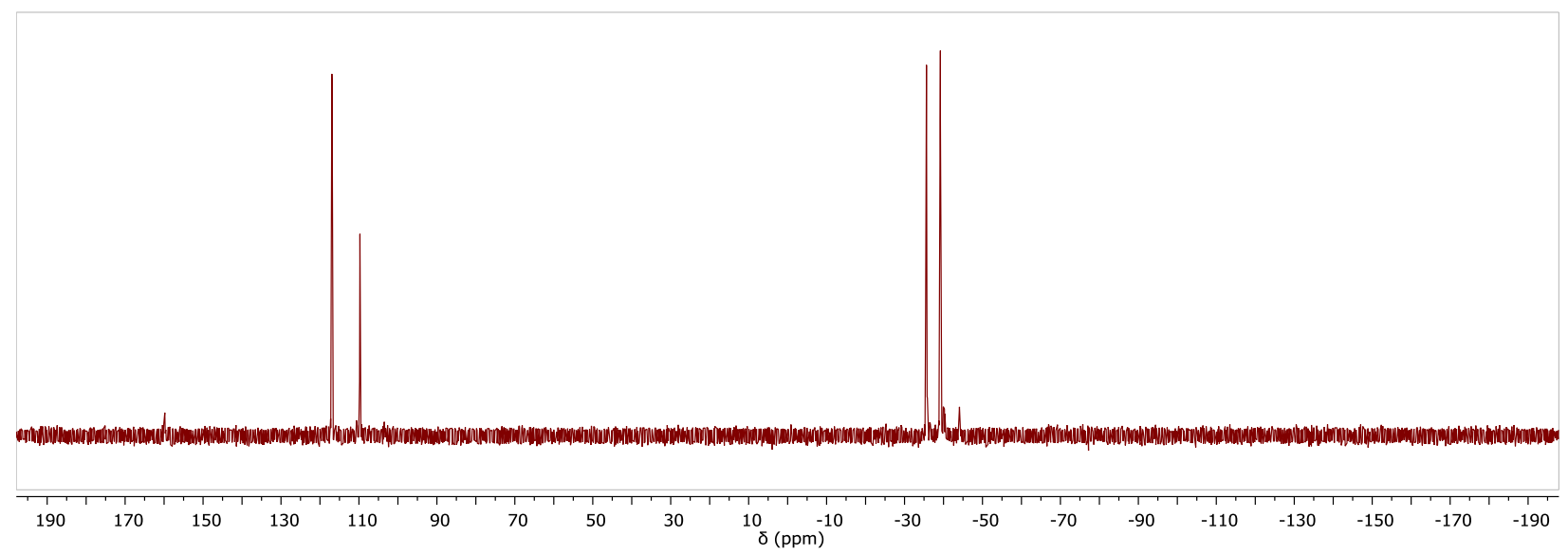

Figure S3. The equilibrium of $\mathbf{1} \cdot[\mathrm{H}]\left[\mathrm{O}^{t} \mathrm{Bu}\right]$ and its tautomers at ambient temperature. 


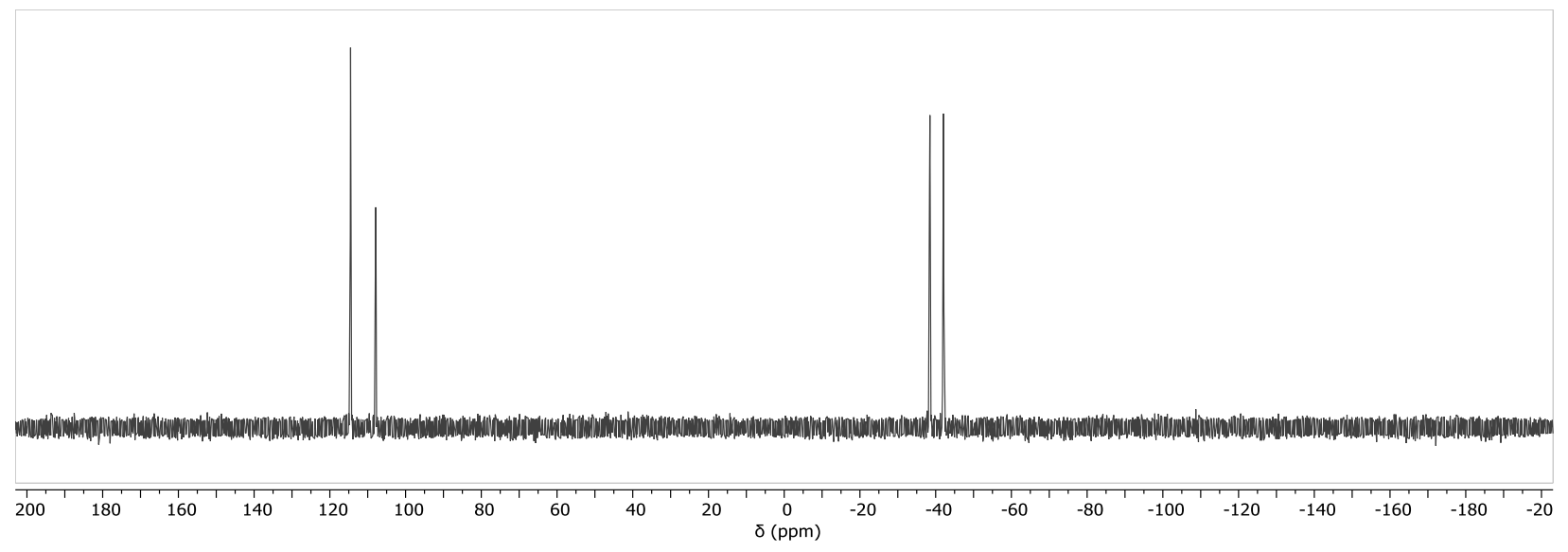

Figure S4. The equilibrium of $\mathbf{1}[\mathrm{H}][\mathrm{O}-1-\mathrm{Ad}]$ and its tautomers at ambient temperature.

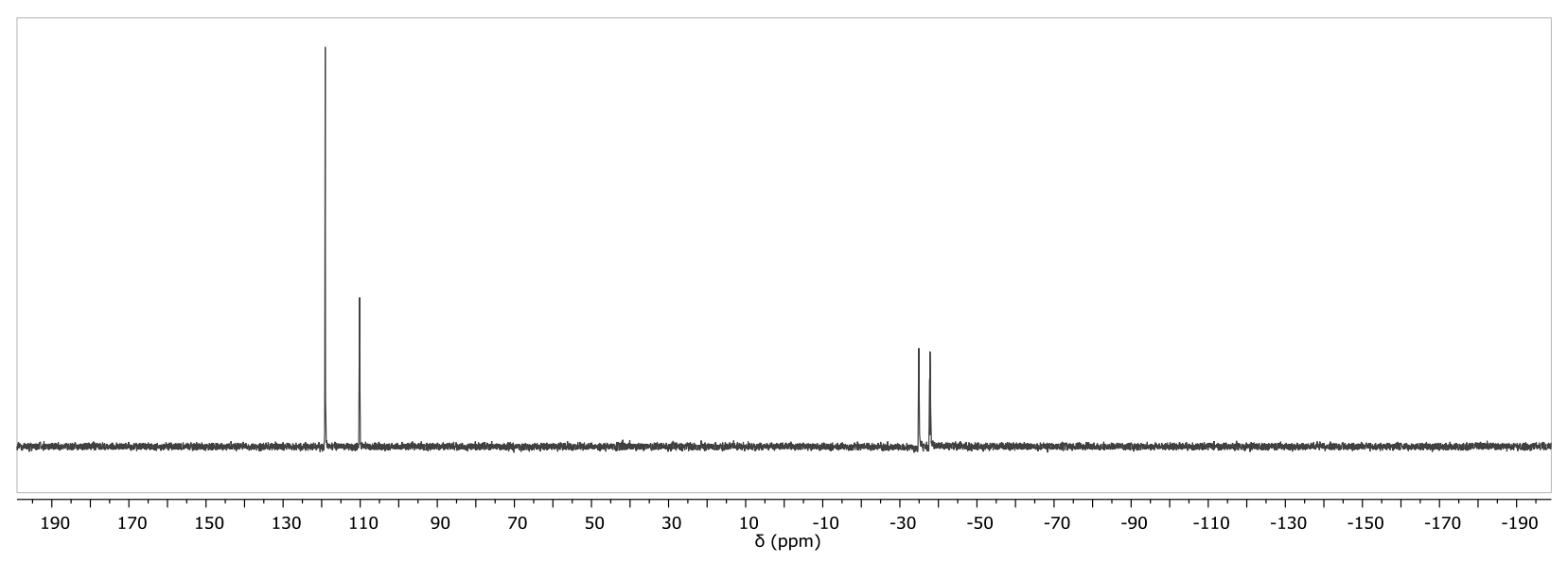

Figure S5. The equilibrium of $\mathbf{1} \cdot[\mathrm{H}]\left[\mathrm{OCPh}_{3}\right]$ and its tautomers at ambient temperature. 


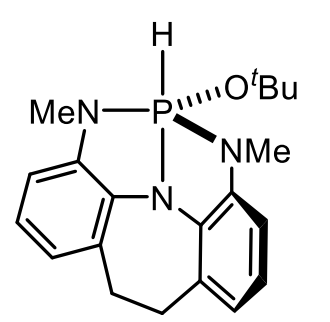

2. $[\mathrm{H}]\left[\mathrm{O}^{t} \mathrm{Bu}\right]$

$\delta-37.7 \mathrm{ppm}$

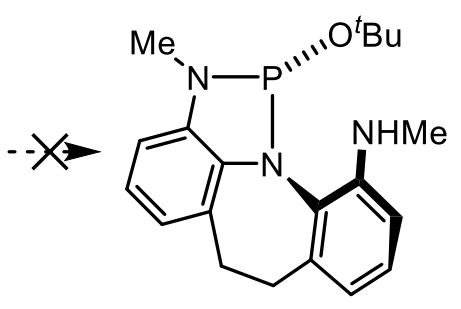

anti- $\sigma^{3}-\mathrm{P}$

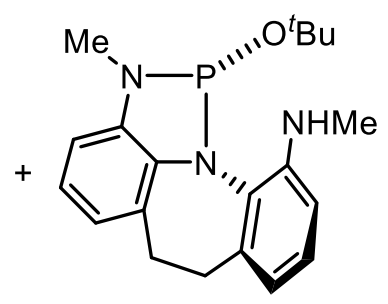

syn- $\sigma^{3}-\mathrm{P}$

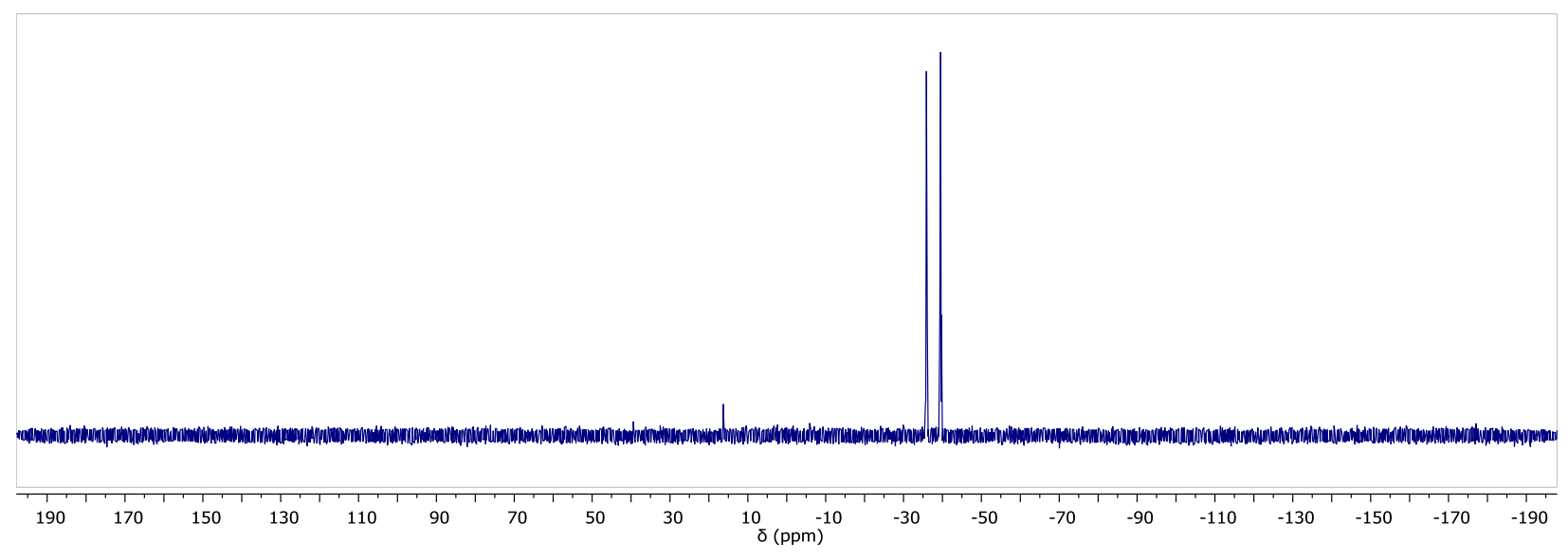

Figure S6. The formation of $2 \cdot[\mathrm{H}]\left[\mathrm{O}^{t} \mathrm{Bu}\right]$ in $15 \mathrm{~min}$ at ambient temperature. 


\section{B. $\mathrm{O}-\mathrm{H}$ bond in carboxylic acids.}

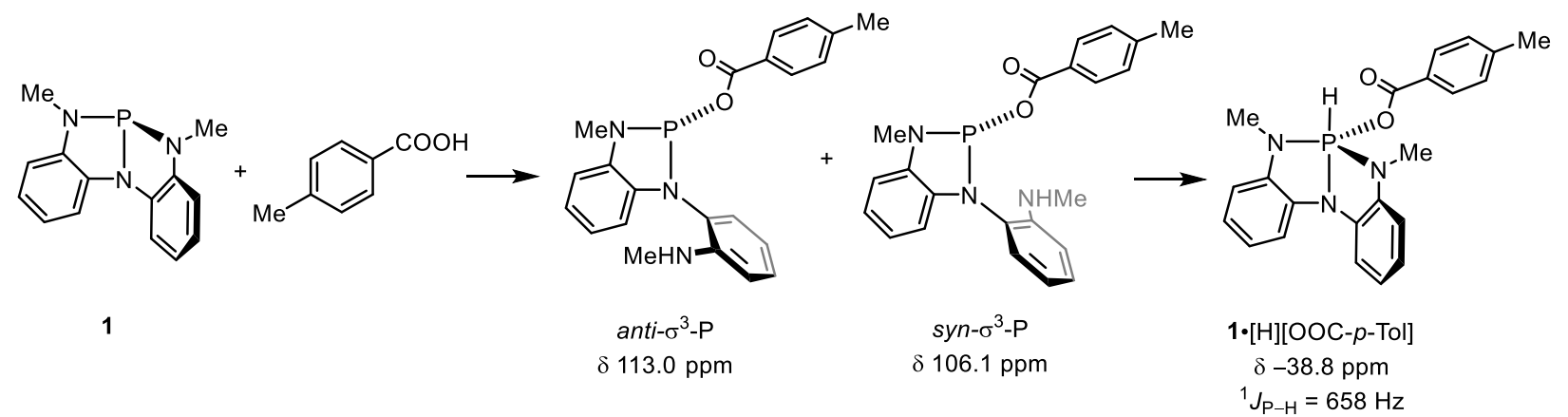

(a)

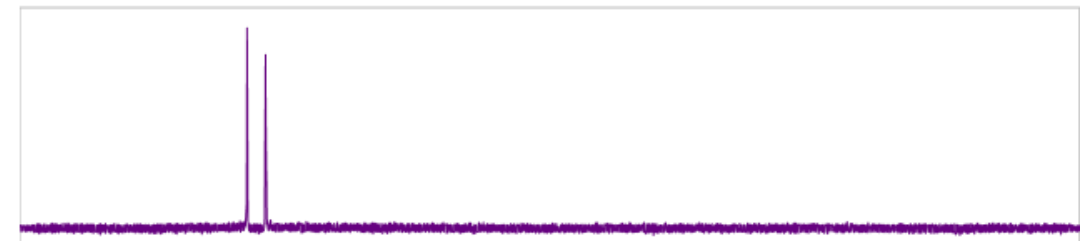

(b)

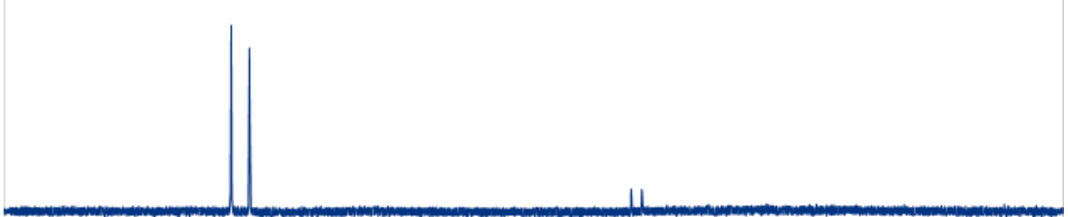

(c)

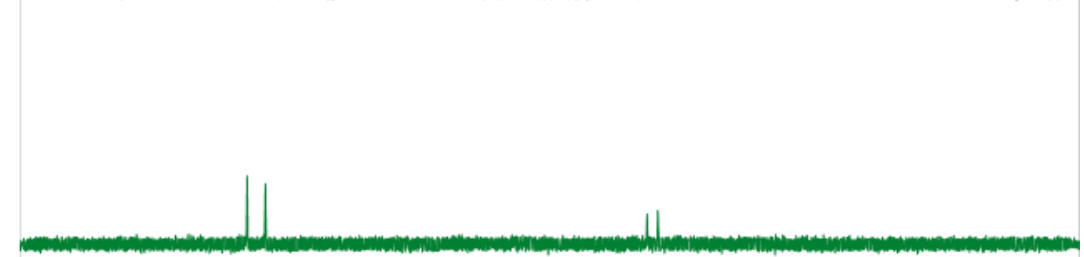

(d)

(e)
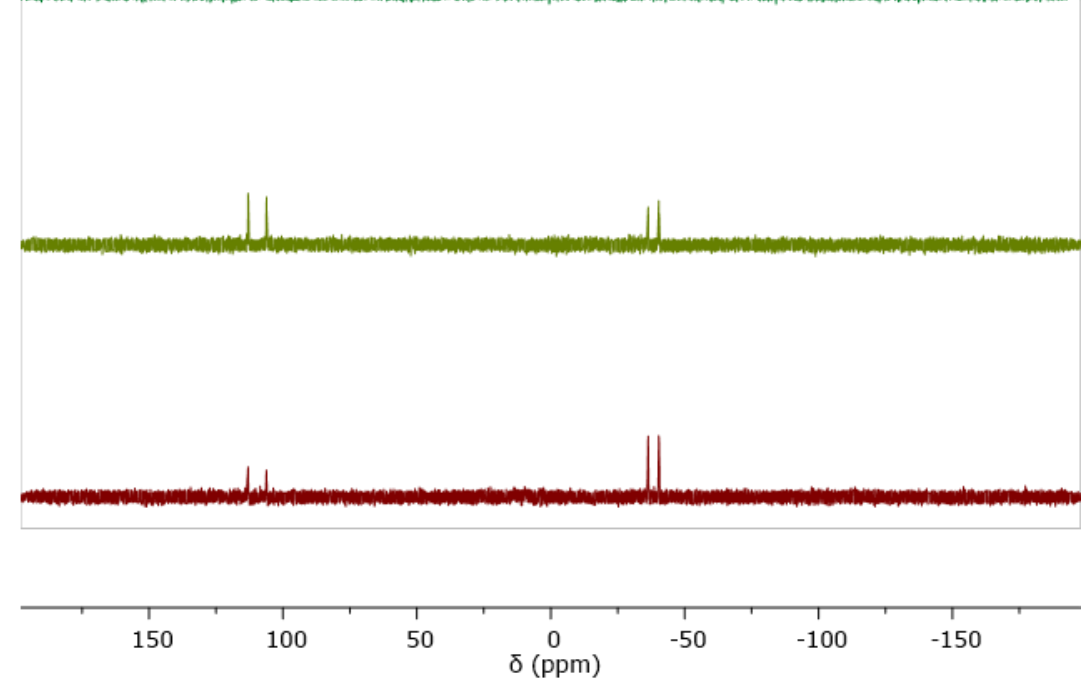

Figure S7. Reaction progress of the formation of $\mathrm{P}(\mathrm{V})$ product $\mathbf{1} \cdot[\mathrm{H}][\mathrm{OOC}-p$-Tol] and the consumption of $\sigma^{3}$-P intermediates as monitored by ${ }^{31} \mathrm{P}$ NMR spectroscopy at ambient temperature in $\mathrm{CDCl}_{3}$. (a) Formation of $\sigma^{3}$-P intermediates, $15 \mathrm{~min}$; (b) $2 \mathrm{~h}$; (c) $8 \mathrm{~h}$; (d) $24 \mathrm{~h}$; (e) $48 \mathrm{~h}$. 

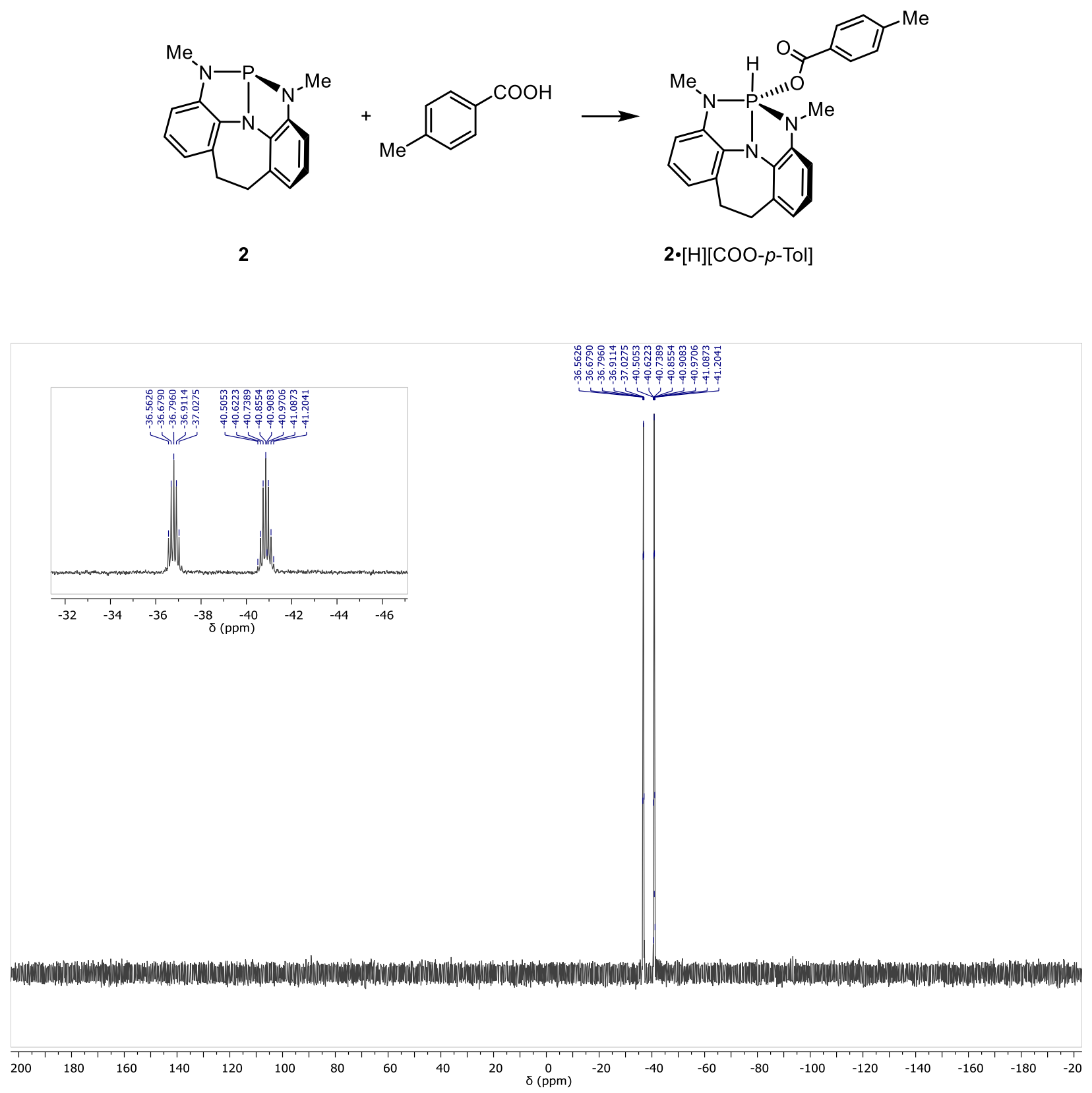

Figure S8. The ${ }^{31} \mathrm{P}$ NMR spectrum of the formation of $\mathbf{2} \cdot[\mathrm{H}][\mathrm{OOC}-p$-Tol] from compound $\mathbf{2}$ and $p$-toluic acid in $15 \mathrm{~min}$ at ambient temperature. 


\section{S-H bonds in thiols.}
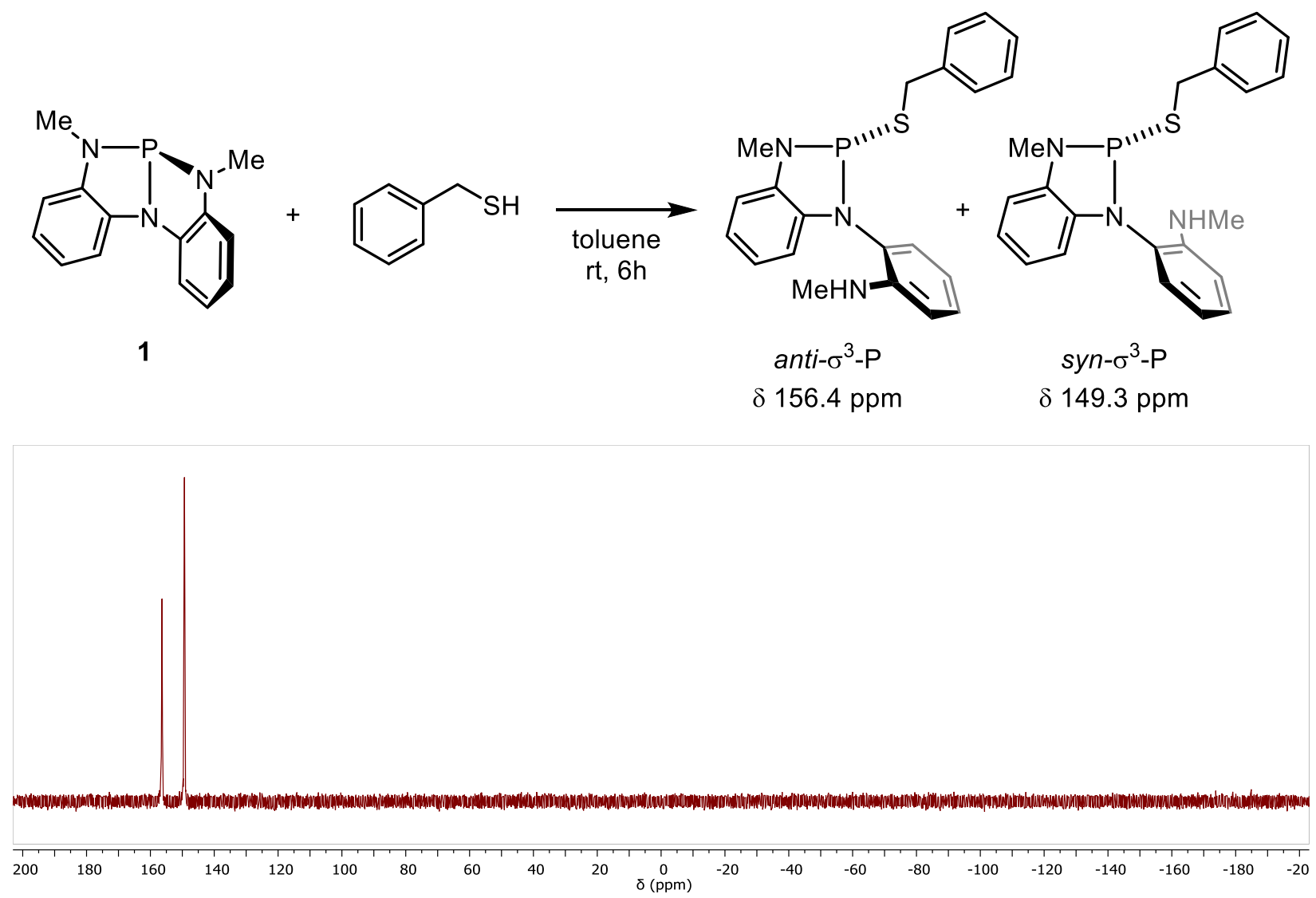

Figure S9. The ${ }^{31} \mathrm{P}$ NMR spectrum of the reaction of compound $\mathbf{1}$ and benzyl mercaptan at ambient temperature. 

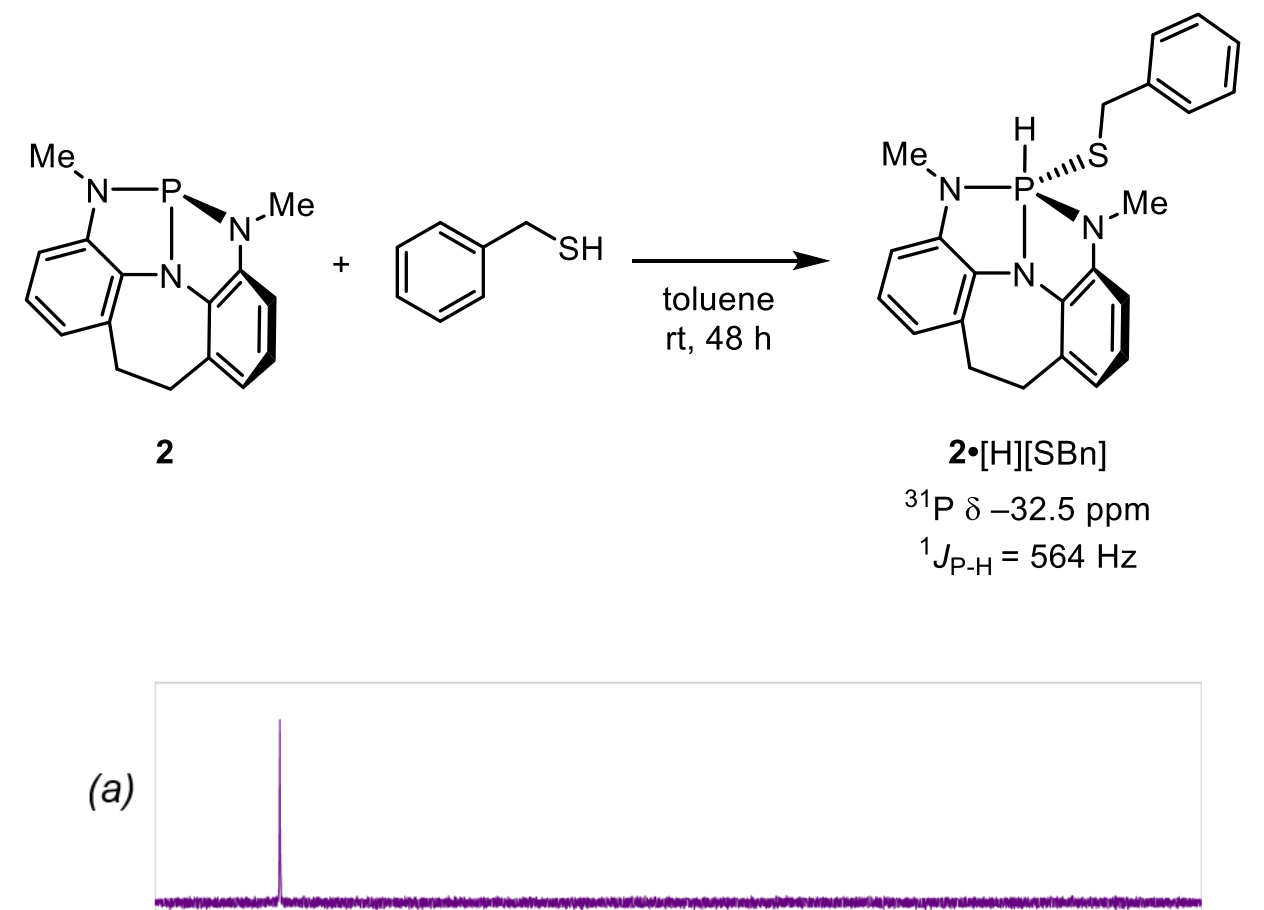

(b)

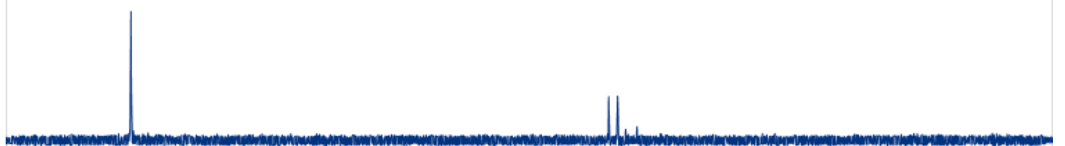

(c)

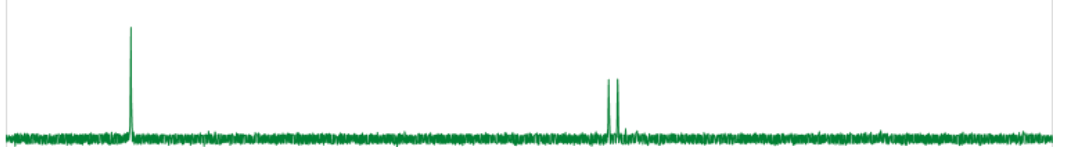

(d)

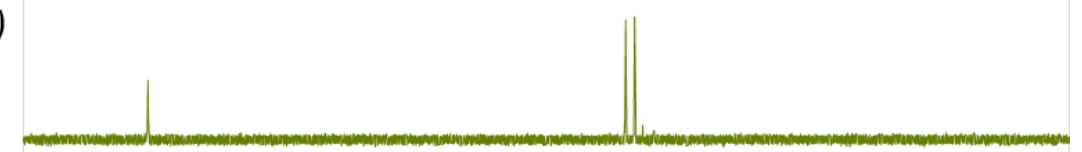

(e)
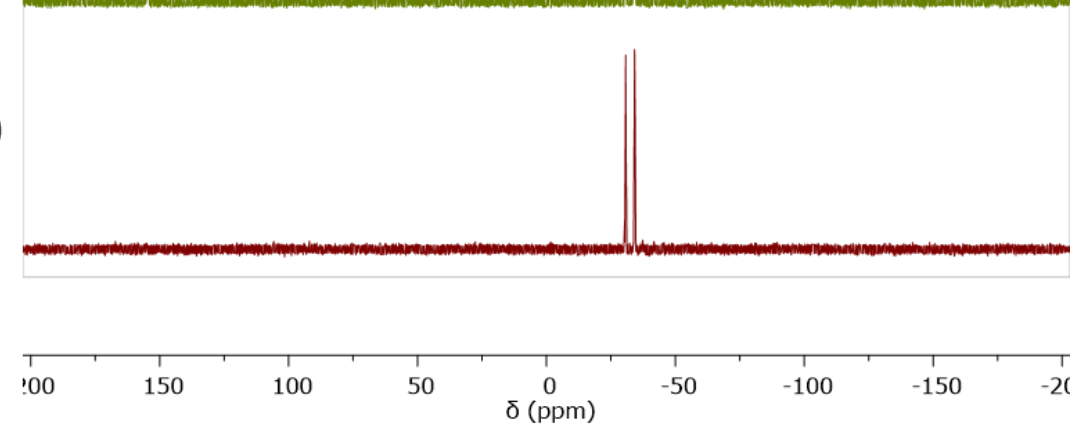

Figure S10. Reaction progress of the formation of $\mathrm{P}(\mathrm{V})$ product $\mathbf{2} \cdot[\mathrm{H}][\mathrm{SBn}]$ and the consumption of $\mathbf{2}$ as monitored by ${ }^{31} \mathrm{P}$ NMR spectroscopy at ambient temperature in toluene- $d_{8}$. (a) $15 \mathrm{~min}$; (b) $2 \mathrm{~h}$; (c) $8 \mathrm{~h}$; (d) $24 \mathrm{~h}$; (e) $48 \mathrm{~h}$. 


\section{Crystallographic Data}

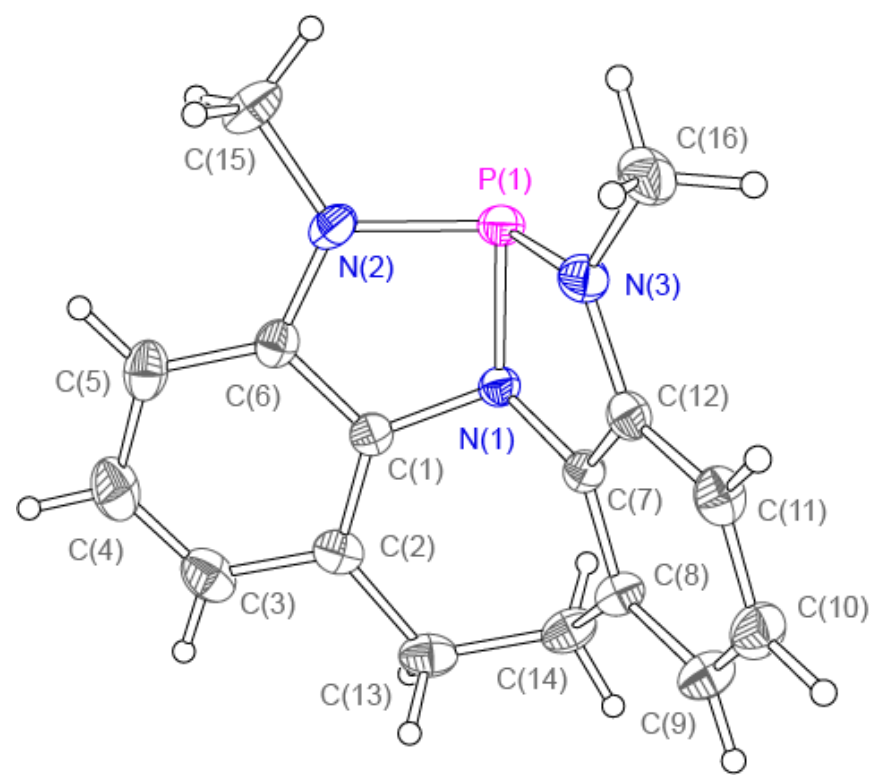

Figure S11. Thermal ellipsoid plot of $\mathbf{2}$ at $50 \%$ probability level.

Table S2. Crystal data and structure refinement for 2 (CCDC 2045988).

\begin{tabular}{|c|c|c|}
\hline Empirical formula & \multicolumn{2}{|l|}{ C16 H16 N3 P } \\
\hline Formula weight & \multicolumn{2}{|l|}{281.29} \\
\hline Temperature & \multicolumn{2}{|l|}{$100(2) \mathrm{K}$} \\
\hline Wavelength & \multicolumn{2}{|l|}{$0.71073 \AA$} \\
\hline Crystal system & \multicolumn{2}{|l|}{ Monoclinic } \\
\hline Space group & \multicolumn{2}{|l|}{$\mathrm{P} 22_{1} / \mathrm{n}$} \\
\hline Unit cell dimensions & $\begin{array}{l}a=6.9209(4) \AA \\
b=10.6388(7) \AA \\
c=18.8310(12) \AA\end{array}$ & $\begin{array}{l}\mathrm{a}=90^{\circ} . \\
\mathrm{b}=97.7460(11)^{\circ} . \\
\mathrm{g}=90^{\circ} .\end{array}$ \\
\hline Volume & $1373.88(15) \AA^{3}$ & \\
\hline $\mathrm{Z}$ & 4 & \\
\hline Density (calculated) & $1.360 \mathrm{Mg} / \mathrm{m}^{3}$ & \\
\hline Absorption coefficient & $0.193 \mathrm{~mm}^{-1}$ & \\
\hline $\mathrm{F}(000)$ & 592 & \\
\hline Crystal size & $0.411 \times 0.187 \times 0.1$ & \\
\hline Theta range for data collection & 2.183 to $31.506^{\circ}$ & \\
\hline Index ranges & $-10<=\mathrm{h}<=10,-15<$ & $-27<=1<=27$ \\
\hline Reflections collected & 38727 & \\
\hline Independent reflections & $4585[\mathrm{R}($ int $)=0.03$ & \\
\hline Completeness to theta $=25.242^{\circ}$ & $99.8 \%$ & \\
\hline Refinement method & Full-matrix least-sc & \\
\hline Data / restraints / parameters & 4585 / 0 / 183 & \\
\hline Goodness-of-fit on $\mathrm{F}^{2}$ & 1.063 & \\
\hline Final $R$ indices $[\mathrm{I}>2 \operatorname{sigma}(\mathrm{I})]$ & $\mathrm{R} 1=0.0368, w \mathrm{R} 2$ & \\
\hline $\mathrm{R}$ indices (all data) & $\mathrm{R} 1=0.0435, \mathrm{wR} 2$ & \\
\hline Largest diff. peak and hole & 0.430 and $-0.264 \mathrm{e}$. & \\
\hline
\end{tabular}


Table S3. Atomic coordinates (x 10 $)$ and equivalent isotropic displacement parameters $\left(\AA^{2} \times 10^{3}\right)$ for 2 . $U(e q)$ is defined as one third of the trace of the orthogonalized $U^{i j}$ tensor.

\begin{tabular}{lrrrr}
\hline & $\mathrm{x}$ & $\mathrm{y}$ & $\mathrm{z}$ & $\mathrm{U}(\mathrm{eq})$ \\
\hline $\mathrm{P}(1)$ & $6015(1)$ & $4337(1)$ & $6592(1)$ & $22(1)$ \\
$\mathrm{N}(1)$ & $4113(1)$ & $5406(1)$ & $6665(1)$ & $19(1)$ \\
$\mathrm{N}(2)$ & $6744(1)$ & $5233(1)$ & $5929(1)$ & $24(1)$ \\
$\mathrm{N}(3)$ & $7051(1)$ & $4709(1)$ & $7452(1)$ & $24(1)$ \\
$\mathrm{C}(1)$ & $4119(2)$ & $6429(1)$ & $6171(1)$ & $20(1)$ \\
$\mathrm{C}(2)$ & $2865(2)$ & $7454(1)$ & $6100(1)$ & $23(1)$ \\
$\mathrm{C}(3)$ & $3187(2)$ & $8348(1)$ & $5575(1)$ & $32(1)$ \\
$\mathrm{C}(4)$ & $4676(2)$ & $8228(1)$ & $5160(1)$ & $35(1)$ \\
$\mathrm{C}(5)$ & $5962(2)$ & $7216(1)$ & $5251(1)$ & $29(1)$ \\
$\mathrm{C}(6)$ & $5668(2)$ & $6321(1)$ & $5761(1)$ & $22(1)$ \\
$\mathrm{C}(7)$ & $4139(2)$ & $5782(1)$ & $7392(1)$ & $19(1)$ \\
$\mathrm{C}(8)$ & $2656(2)$ & $6441(1)$ & $7642(1)$ & $23(1)$ \\
$\mathrm{C}(9)$ & $2925(2)$ & $6776(1)$ & $8370(1)$ & $29(1)$ \\
$\mathrm{C}(10)$ & $4633(2)$ & $6450(1)$ & $8807(1)$ & $30(1)$ \\
$\mathrm{C}(11)$ & $6125(2)$ & $5780(1)$ & $8546(1)$ & $26(1)$ \\
$\mathrm{C}(12)$ & $5857(2)$ & $5438(1)$ & $7826(1)$ & $21(1)$ \\
$\mathrm{C}(13)$ & $1265(2)$ & $7723(1)$ & $6558(1)$ & $29(1)$ \\
$\mathrm{C}(14)$ & $874(2)$ & $6746(1)$ & $7117(1)$ & $27(1)$ \\
$\mathrm{C}(15)$ & $8254(2)$ & $4836(1)$ & $5512(1)$ & $32(1)$ \\
$\mathrm{C}(16)$ & $8664(2)$ & $3987(1)$ & $7827(1)$ & $31(1)$ \\
\hline
\end{tabular}

Table S4. Bond lengths $[\AA]$ and angles $\left[^{\circ}\right]$ for 2.

\begin{tabular}{ll}
\hline $\mathrm{P}(1)-\mathrm{N}(2)$ & $1.7009(10)$ \\
$\mathrm{P}(1)-\mathrm{N}(3)$ & $1.7259(10)$ \\
$\mathrm{P}(1)-\mathrm{N}(1)$ & $1.7592(9)$ \\
$\mathrm{N}(1)-\mathrm{C}(7)$ & $1.4249(13)$ \\
$\mathrm{N}(1)-\mathrm{C}(1)$ & $1.4307(13)$ \\
$\mathrm{N}(2)-\mathrm{C}(6)$ & $1.3896(15)$ \\
$\mathrm{N}(2)-\mathrm{C}(15)$ & $1.4525(14)$ \\
$\mathrm{N}(3)-\mathrm{C}(12)$ & $1.3912(14)$ \\
$\mathrm{N}(3)-\mathrm{C}(16)$ & $1.4567(14)$ \\
$\mathrm{C}(1)-\mathrm{C}(2)$ & $1.3892(15)$ \\
$\mathrm{C}(1)-\mathrm{C}(6)$ & $1.4079(15)$ \\
$\mathrm{C}(2)-\mathrm{C}(3)$ & $1.4114(17)$ \\
$\mathrm{C}(2)-\mathrm{C}(13)$ & $1.5198(17)$ \\
$\mathrm{C}(3)-\mathrm{C}(4)$ & $1.380(2)$ \\
$\mathrm{C}(3)-\mathrm{H}(3)$ & 0.9500 \\
$\mathrm{C}(4)-\mathrm{C}(5)$ & $1.3933(19)$ \\
$\mathrm{C}(4)-\mathrm{H}(4)$ & 0.9500 \\
$\mathrm{C}(5)-\mathrm{C}(6)$ & $1.3871(16)$ \\
$\mathrm{C}(5)-\mathrm{H}(5)$ & 0.9500 \\
$\mathrm{C}(7)-\mathrm{C}(8)$ & $1.3770(14)$ \\
&
\end{tabular}




$\begin{array}{ll}\mathrm{C}(7)-\mathrm{C}(12) & 1.3984(14) \\ \mathrm{C}(8)-\mathrm{C}(9) & 1.4046(15) \\ \mathrm{C}(8)-\mathrm{C}(14) & 1.5073(15) \\ \mathrm{C}(9)-\mathrm{C}(10) & 1.3901(17) \\ \mathrm{C}(9)-\mathrm{H}(9) & 0.9500 \\ \mathrm{C}(10)-\mathrm{C}(11) & 1.3975(18) \\ \mathrm{C}(10)-\mathrm{H}(10) & 0.9500 \\ \mathrm{C}(11)-\mathrm{C}(12) & 1.3920(15) \\ \mathrm{C}(11)-\mathrm{H}(11) & 0.9500 \\ \mathrm{C}(13)-\mathrm{C}(14) & 1.5293(18) \\ \mathrm{C}(13)-\mathrm{H}(13 \mathrm{~A}) & 0.9900 \\ \mathrm{C}(13)-\mathrm{H}(13 \mathrm{~B}) & 0.9900 \\ \mathrm{C}(14)-\mathrm{H}(14 \mathrm{~A}) & 0.9900 \\ \mathrm{C}(14)-\mathrm{H}(14 \mathrm{~B}) & 0.9900 \\ \mathrm{C}(15)-\mathrm{H}(15 \mathrm{~A}) & 0.9800 \\ \mathrm{C}(15)-\mathrm{H}(15 \mathrm{~B}) & 0.9800 \\ \mathrm{C}(15)-\mathrm{H}(15 \mathrm{C}) & 0.9800 \\ \mathrm{C}(16)-\mathrm{H}(16 \mathrm{~A}) & 0.9800 \\ \mathrm{C}(16)-\mathrm{H}(16 \mathrm{~B}) & 0.9800 \\ \mathrm{C}(16)-\mathrm{H}(16 \mathrm{C}) & 0.9800 \\ \end{array}$

Table S5. Bond angles $\left[{ }^{\circ}\right]$ for 2.

\begin{tabular}{ll}
\hline $\mathrm{N}(2)-\mathrm{P}(1)-\mathrm{N}(3)$ & $115.68(5)$ \\
$\mathrm{N}(2)-\mathrm{P}(1)-\mathrm{N}(1)$ & $89.60(5)$ \\
$\mathrm{N}(3)-\mathrm{P}(1)-\mathrm{N}(1)$ & $89.99(4)$ \\
$\mathrm{C}(7)-\mathrm{N}(1)-\mathrm{C}(1)$ & $114.19(8)$ \\
$\mathrm{C}(7)-\mathrm{N}(1)-\mathrm{P}(1)$ & $110.07(7)$ \\
$\mathrm{C}(1)-\mathrm{N}(1)-\mathrm{P}(1)$ & $111.96(7)$ \\
$\mathrm{C}(6)-\mathrm{N}(2)-\mathrm{C}(15)$ & $121.42(10)$ \\
$\mathrm{C}(6)-\mathrm{N}(2)-\mathrm{P}(1)$ & $115.57(7)$ \\
$\mathrm{C}(15)-\mathrm{N}(2)-\mathrm{P}(1)$ & $122.71(9)$ \\
$\mathrm{C}(12)-\mathrm{N}(3)-\mathrm{C}(16)$ & $121.15(10)$ \\
$\mathrm{C}(12)-\mathrm{N}(3)-\mathrm{P}(1)$ & $113.75(7)$ \\
$\mathrm{C}(16)-\mathrm{N}(3)-\mathrm{P}(1)$ & $121.85(9)$ \\
$\mathrm{C}(2)-\mathrm{C}(1)-\mathrm{C}(6)$ & $121.52(10)$ \\
$\mathrm{C}(2)-\mathrm{C}(1)-\mathrm{N}(1)$ & $127.11(10)$ \\
$\mathrm{C}(6)-\mathrm{C}(1)-\mathrm{N}(1)$ & $111.31(9)$ \\
$\mathrm{C}(1)-\mathrm{C}(2)-\mathrm{C}(3)$ & $116.17(11)$ \\
$\mathrm{C}(1)-\mathrm{C}(2)-\mathrm{C}(13)$ & $125.89(10)$ \\
$\mathrm{C}(3)-\mathrm{C}(2)-\mathrm{C}(13)$ & $117.87(10)$ \\
$\mathrm{C}(4)-\mathrm{C}(3)-\mathrm{C}(2)$ & $122.38(11)$ \\
$\mathrm{C}(4)-\mathrm{C}(3)-\mathrm{H}(3)$ & 118.8 \\
$\mathrm{C}(2)-\mathrm{C}(3)-\mathrm{H}(3)$ & 118.8 \\
$\mathrm{C}(3)-\mathrm{C}(4)-\mathrm{C}(5)$ & $120.97(11)$ \\
$\mathrm{C}(3)-\mathrm{C}(4)-\mathrm{H}(4)$ & 119.5 \\
$\mathrm{C}(5)-\mathrm{C}(4)-\mathrm{H}(4)$ & 119.5 \\
$\mathrm{C}(6)-\mathrm{C}(5)-\mathrm{C}(4)$ & $117.71(12)$ \\
$\mathrm{C}(6)-\mathrm{C}(5)-\mathrm{H}(5)$ & 121.1 \\
$\mathrm{C}(4)-\mathrm{C}(5)-\mathrm{H}(5)$ & 121.1
\end{tabular}




$\begin{array}{ll}\mathrm{C}(5)-\mathrm{C}(6)-\mathrm{N}(2) & 127.25(11) \\ \mathrm{C}(5)-\mathrm{C}(6)-\mathrm{C}(1) & 121.20(11) \\ \mathrm{N}(2)-\mathrm{C}(6)-\mathrm{C}(1) & 111.53(9) \\ \mathrm{C}(8)-\mathrm{C}(7)-\mathrm{C}(12) & 123.24(10) \\ \mathrm{C}(8)-\mathrm{C}(7)-\mathrm{N}(1) & 123.92(9) \\ \mathrm{C}(12)-\mathrm{C}(7)-\mathrm{N}(1) & 112.83(9) \\ \mathrm{C}(7)-\mathrm{C}(8)-\mathrm{C}(9) & 117.09(10) \\ \mathrm{C}(7)-\mathrm{C}(8)-\mathrm{C}(14) & 117.86(9) \\ \mathrm{C}(9)-\mathrm{C}(8)-\mathrm{C}(14) & 125.05(10) \\ \mathrm{C}(10)-\mathrm{C}(9)-\mathrm{C}(8) & 120.39(11) \\ \mathrm{C}(10)-\mathrm{C}(9)-\mathrm{H}(9) & 119.8 \\ \mathrm{C}(8)-\mathrm{C}(9)-\mathrm{H}(9) & 119.8 \\ \mathrm{C}(9)-\mathrm{C}(10)-\mathrm{C}(11) & 121.85(10) \\ \mathrm{C}(9)-\mathrm{C}(10)-\mathrm{H}(10) & 119.1 \\ \mathrm{C}(11)-\mathrm{C}(10)-\mathrm{H}(10) & 119.1 \\ \mathrm{C}(12)-\mathrm{C}(11)-\mathrm{C}(10) & 117.97(10) \\ \mathrm{C}(12)-\mathrm{C}(11)-\mathrm{H}(11) & 121.0 \\ \mathrm{C}(10)-\mathrm{C}(11)-\mathrm{H}(11) & 121.0 \\ \mathrm{~N}(3)-\mathrm{C}(12)-\mathrm{C}(11) & 128.96(10) \\ \mathrm{N}(3)-\mathrm{C}(12)-\mathrm{C}(7) & 111.50(9) \\ \mathrm{C}(11)-\mathrm{C}(12)-\mathrm{C}(7) & 119.45(10) \\ \mathrm{C}(2)-\mathrm{C}(13)-\mathrm{C}(14) & 118.24(10) \\ \mathrm{C}(2)-\mathrm{C}(13)-\mathrm{H}(13 \mathrm{~A}) & 107.7 \\ \mathrm{C}(14)-\mathrm{C}(13)-\mathrm{H}(13 \mathrm{~A}) & 107.7 \\ \mathrm{C}(2)-\mathrm{C}(13)-\mathrm{H}(13 \mathrm{~B}) & 107.7 \\ \mathrm{C}(14)-\mathrm{C}(13)-\mathrm{H}(13 \mathrm{~B}) & 107.7 \\ \mathrm{H}(13 \mathrm{~A})-\mathrm{C}(13)-\mathrm{H}(13 \mathrm{~B}) & 107.1 \\ \mathrm{C}(8)-\mathrm{C}(14)-\mathrm{C}(13) & 113.07(9) \\ \mathrm{C}(8)-\mathrm{C}(14)-\mathrm{H}(14 \mathrm{~A}) & 109.0 \\ \mathrm{C}(13)-\mathrm{C}(14)-\mathrm{H}(14 \mathrm{~A}) & 109.0 \\ \mathrm{C}(8)-\mathrm{C}(14)-\mathrm{H}(14 \mathrm{~B}) & 109.0 \\ \mathrm{C}(13)-\mathrm{C}(14)-\mathrm{H}(14 \mathrm{~B}) & 109.0 \\ \mathrm{H}(14 \mathrm{~A})-\mathrm{C}(14)-\mathrm{H}(14 \mathrm{~B}) & 107.8 \\ \mathrm{~N}(2)-\mathrm{C}(15)-\mathrm{H}(15 \mathrm{~A}) & 109.5 \\ \mathrm{~N}(2)-\mathrm{C}(15)-\mathrm{H}(15 \mathrm{~B}) & 109.5 \\ \mathrm{H}(15 \mathrm{~A})-\mathrm{C}(15)-\mathrm{H}(15 \mathrm{~B}) & 109.5 \\ \mathrm{~N}(2)-\mathrm{C}(15)-\mathrm{H}(15 \mathrm{C}) & 109.5 \\ \mathrm{H}(15 \mathrm{~A})-\mathrm{C}(15)-\mathrm{H}(15 \mathrm{C}) & 109.5 \\ \mathrm{H}(15 \mathrm{~B})-\mathrm{C}(15)-\mathrm{H}(15 \mathrm{C}) & 109.5 \\ \mathrm{~N}(3)-\mathrm{C}(16)-\mathrm{H}(16 \mathrm{~A}) & 109.5 \\ \mathrm{~N}(3)-\mathrm{C}(16)-\mathrm{H}(16 \mathrm{~B}) & 109.5 \\ \mathrm{H}(16 \mathrm{~A})-\mathrm{C}(16)-\mathrm{H}(16 \mathrm{~B}) & 109.5 \\ \mathrm{~N}(3)-\mathrm{C}(16)-\mathrm{H}(16 \mathrm{C}) & 109.5 \\ \mathrm{H}(16 \mathrm{~A})-\mathrm{C}(16)-\mathrm{H}(16 \mathrm{C}) & 109.5 \\ \mathrm{H}(16 \mathrm{~B})-\mathrm{C}(16)-\mathrm{H}(16 \mathrm{C}) & 109.5 \\ & \end{array}$




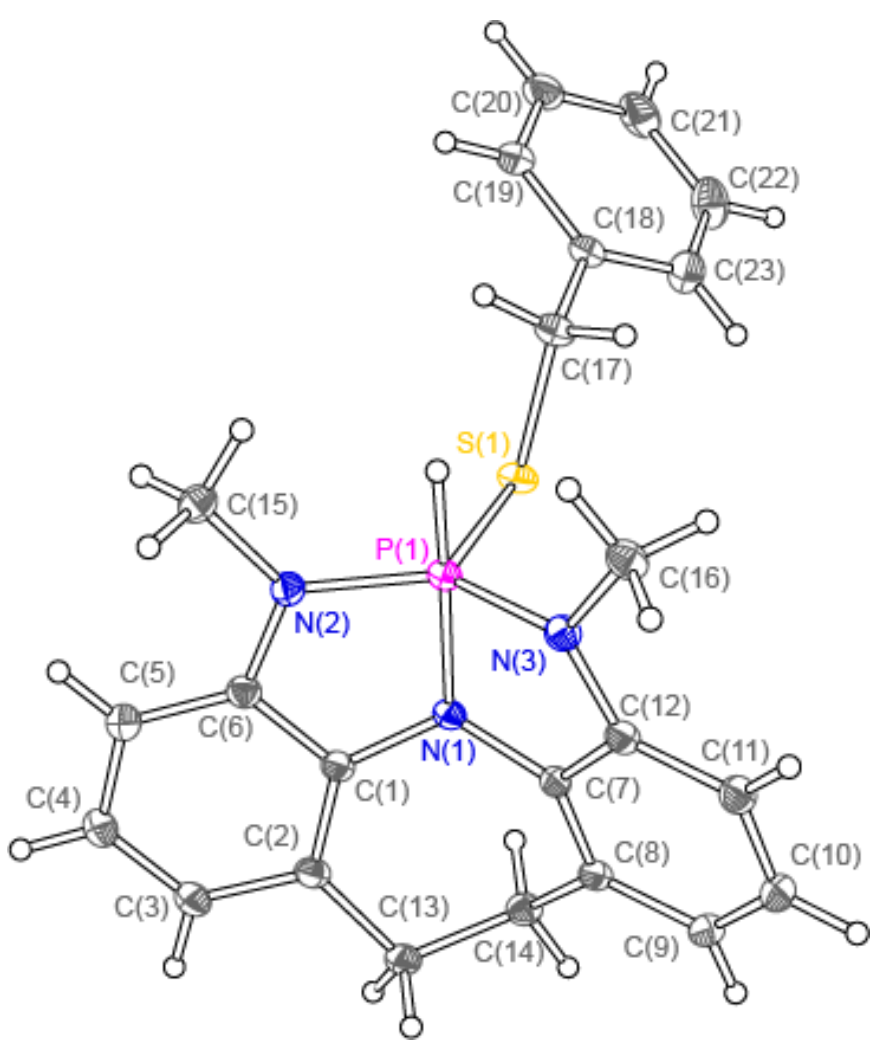

Figure S12. Thermal ellipsoid plot of a $\mathbf{2} \cdot[\mathrm{H}][\mathrm{SBn}]$ dimer at $50 \%$ probability level.

Table S6. Crystal data and structure refinement for $2 \cdot[\mathrm{H}][\mathrm{SBn}]$ (CCDC 2045992).

\begin{tabular}{lll}
\hline Empirical formula & $\mathrm{C} 23 \mathrm{H} 24 \mathrm{~N} 3 \mathrm{P} \mathrm{S}$ & \\
Formula weight & 405.48 & \\
Temperature & $100(2) \mathrm{K}$ & \\
Wavelength & $0.71073 \AA$ & \\
Crystal system & Orthorhombic & \\
Space group & $\mathrm{P} 22_{1} 21$ & $\mathrm{a}=90^{\circ}$. \\
Unit cell dimensions & $\mathrm{a}=5.7007(2) \AA$ & $\mathrm{b}=90^{\circ}$. \\
& $\mathrm{b}=14.1105(4) \AA$ & $\mathrm{g}=90^{\circ}$. \\
& $\mathrm{c}=24.3538(8) \AA$ & \\
Volume & $1959.01(11) \AA^{3}$ & \\
Z & 4 & \\
Density (calculated) & $1.375 \mathrm{Mg} / \mathrm{m}^{3}$ & \\
Absorption coefficient & $0.261 \mathrm{~mm}-1$ & \\
F(000) & 856 & \\
Crystal size & $0.520 \times 0.110 \times 0.045 \mathrm{~mm}^{3}$ \\
Theta range for data collection & 1.668 to $36.450^{\circ}$. \\
Index ranges & $-9<=\mathrm{h}<=9,-23<=\mathrm{k}<=23,-40<=1<=40$ \\
Reflections collected & 151487 & \\
Independent reflections & $9571[\mathrm{R}(\mathrm{int})=0.0573]$ & \\
Completeness to theta $=25.242^{\circ}$ & $100.0 \%$ & \\
\hline
\end{tabular}




\begin{tabular}{ll}
\hline Refinement method & Full-matrix least-squares on $\mathrm{F}^{2}$ \\
Data / restraints / parameters & $9571 / 0 / 258$ \\
Goodness-of-fit on F & 1.037 \\
Final R indices [I>2sigma(I)] & $\mathrm{R} 1=0.0348, \mathrm{wR} 2=0.0783$ \\
R indices (all data) & $\mathrm{R} 1=0.0412, \mathrm{wR} 2=0.0828$ \\
Absolute structure parameter & $0.018(12)$ \\
Extinction coefficient & $\mathrm{n} / \mathrm{a}$ \\
Largest diff. peak and hole & 0.332 and -0.470 e. $\AA^{-3}$
\end{tabular}

Table S7. Atomic coordinates (x 10 $)$ and equivalent isotropic displacement parameters $\left(\AA^{2} \times 10^{3}\right)$ for $2 \cdot[\mathrm{H}][\mathrm{SBn}]$. $\mathrm{U}(\mathrm{eq})$ is defined as one third of the trace of the orthogonalized $\mathrm{Uij}^{\mathrm{ij}}$ tensor.

\begin{tabular}{lrrrr}
\hline & $\mathrm{x}$ & $\mathrm{y}$ & $\mathrm{z}$ & $\mathrm{U}(\mathrm{eq})$ \\
\hline $\mathrm{P}(1)$ & $4574(1)$ & $5512(1)$ & $2249(1)$ & $13(1)$ \\
$\mathrm{S}(1)$ & $7218(1)$ & $5636(1)$ & $2867(1)$ & $16(1)$ \\
$\mathrm{N}(1)$ & $6672(2)$ & $5545(1)$ & $1707(1)$ & $13(1)$ \\
$\mathrm{N}(2)$ & $3581(2)$ & $6568(1)$ & $2004(1)$ & $14(1)$ \\
$\mathrm{N}(3)$ & $4113(2)$ & $4347(1)$ & $2090(1)$ & $15(1)$ \\
$\mathrm{C}(1)$ & $6496(3)$ & $6325(1)$ & $1354(1)$ & $14(1)$ \\
$\mathrm{C}(2)$ & $7733(3)$ & $6528(1)$ & $874(1)$ & $15(1)$ \\
$\mathrm{C}(3)$ & $7172(3)$ & $7384(1)$ & $607(1)$ & $20(1)$ \\
$\mathrm{C}(4)$ & $5421(3)$ & $7983(1)$ & $795(1)$ & $22(1)$ \\
$\mathrm{C}(5)$ & $4133(3)$ & $7754(1)$ & $1266(1)$ & $19(1)$ \\
$\mathrm{C}(6)$ & $4705(3)$ & $6929(1)$ & $1542(1)$ & $15(1)$ \\
$\mathrm{C}(7)$ & $7339(2)$ & $4638(1)$ & $1537(1)$ & $13(1)$ \\
$\mathrm{C}(8)$ & $9246(2)$ & $4417(1)$ & $1208(1)$ & $14(1)$ \\
$\mathrm{C}(9)$ & $9626(3)$ & $3454(1)$ & $1095(1)$ & $17(1)$ \\
$\mathrm{C}(10)$ & $8142(3)$ & $2760(1)$ & $1306(1)$ & $18(1)$ \\
$\mathrm{C}(11)$ & $6218(3)$ & $2995(1)$ & $1636(1)$ & $17(1)$ \\
$\mathrm{C}(12)$ & $5844(3)$ & $3947(1)$ & $1757(1)$ & $14(1)$ \\
$\mathrm{C}(13)$ & $9564(3)$ & $5892(1)$ & $612(1)$ & $17(1)$ \\
$\mathrm{C}(14)$ & $10809(3)$ & $5197(1)$ & $1000(1)$ & $16(1)$ \\
$\mathrm{C}(15)$ & $1549(3)$ & $7074(1)$ & $2216(1)$ & $17(1)$ \\
$\mathrm{C}(16)$ & $2520(3)$ & $3734(1)$ & $2393(1)$ & $18(1)$ \\
$\mathrm{C}(17)$ & $5585(3)$ & $5369(1)$ & $3497(1)$ & $17(1)$ \\
$\mathrm{C}(18)$ & $7127(3)$ & $5574(1)$ & $3988(1)$ & $16(1)$ \\
$\mathrm{C}(19)$ & $6569(3)$ & $6317(1)$ & $4342(1)$ & $19(1)$ \\
$\mathrm{C}(20)$ & $7966(3)$ & $6520(1)$ & $4794(1)$ & $23(1)$ \\
$\mathrm{C}(21)$ & $9947(3)$ & $5980(1)$ & $4899(1)$ & $26(1)$ \\
$\mathrm{C}(22)$ & $10524(4)$ & $5235(1)$ & $4550(1)$ & $27(1)$ \\
& & & &
\end{tabular}


Table S8. Bond lengths $[\AA]$ for $\mathbf{2} \cdot[\mathrm{H}][\mathrm{SBn}]$.

\begin{tabular}{|c|c|}
\hline $\mathrm{P}(1)-\mathrm{N}(2)$ & $1.7021(13)$ \\
\hline $\mathrm{P}(1)-\mathrm{N}(3)$ & $1.7088(13)$ \\
\hline $\mathrm{P}(1)-\mathrm{N}(1)$ & $1.7830(11)$ \\
\hline $\mathrm{P}(1)-\mathrm{S}(1)$ & $2.1369(5)$ \\
\hline $\mathrm{P}(1)-\mathrm{H}(1)$ & $1.36(2)$ \\
\hline $\mathrm{S}(1)-\mathrm{C}(17)$ & $1.8335(14)$ \\
\hline $\mathrm{N}(1)-\mathrm{C}(7)$ & $1.3977(18)$ \\
\hline $\mathrm{N}(1)-\mathrm{C}(1)$ & $1.3995(18)$ \\
\hline $\mathrm{N}(2)-\mathrm{C}(6)$ & $1.3925(18)$ \\
\hline $\mathrm{N}(2)-\mathrm{C}(15)$ & $1.4554(19)$ \\
\hline $\mathrm{N}(3)-\mathrm{C}(12)$ & $1.3972(18)$ \\
\hline$N(3)-C(16)$ & $1.4562(18)$ \\
\hline$C(1)-C(2)$ & $1.3959(19)$ \\
\hline $\mathrm{C}(1)-\mathrm{C}(6)$ & $1.406(2)$ \\
\hline $\mathrm{C}(2)-\mathrm{C}(3)$ & $1.408(2)$ \\
\hline$C(2)-C(13)$ & $1.516(2)$ \\
\hline$C(3)-C(4)$ & $1.386(2)$ \\
\hline $\mathrm{C}(3)-\mathrm{H}(3)$ & 0.9500 \\
\hline$C(4)-C(5)$ & $1.398(2)$ \\
\hline $\mathrm{C}(4)-\mathrm{H}(4)$ & 0.9500 \\
\hline$C(5)-C(6)$ & $1.384(2)$ \\
\hline $\mathrm{C}(5)-\mathrm{H}(5)$ & 0.9500 \\
\hline$C(7)-C(8)$ & $1.3867(19)$ \\
\hline $\mathrm{C}(7)-\mathrm{C}(12)$ & $1.4000(19)$ \\
\hline $\mathrm{C}(8)-\mathrm{C}(9)$ & $1.403(2)$ \\
\hline$C(8)-C(14)$ & $1.504(2)$ \\
\hline $\mathrm{C}(9)-\mathrm{C}(10)$ & $1.392(2)$ \\
\hline $\mathrm{C}(9)-\mathrm{H}(9)$ & 0.9500 \\
\hline $\mathrm{C}(10)-\mathrm{C}(11)$ & $1.400(2)$ \\
\hline $\mathrm{C}(10)-\mathrm{H}(10)$ & 0.9500 \\
\hline $\mathrm{C}(11)-\mathrm{C}(12)$ & $1.392(2)$ \\
\hline $\mathrm{C}(11)-\mathrm{H}(11)$ & 0.9500 \\
\hline$C(13)-C(14)$ & $1.535(2)$ \\
\hline $\mathrm{C}(13)-\mathrm{H}(13 \mathrm{~A})$ & 0.9900 \\
\hline $\mathrm{C}(13)-\mathrm{H}(13 \mathrm{~B})$ & 0.9900 \\
\hline $\mathrm{C}(14)-\mathrm{H}(14 \mathrm{~A})$ & 0.9900 \\
\hline $\mathrm{C}(14)-\mathrm{H}(14 \mathrm{~B})$ & 0.9900 \\
\hline $\mathrm{C}(15)-\mathrm{H}(15 \mathrm{~A})$ & 0.9800 \\
\hline $\mathrm{C}(15)-\mathrm{H}(15 \mathrm{~B})$ & 0.9800 \\
\hline $\mathrm{C}(15)-\mathrm{H}(15 \mathrm{C})$ & 0.9800 \\
\hline $\mathrm{C}(16)-\mathrm{H}(16 \mathrm{~A})$ & 0.9800 \\
\hline $\mathrm{C}(16)-\mathrm{H}(16 \mathrm{~B})$ & 0.9800 \\
\hline $\mathrm{C}(16)-\mathrm{H}(16 \mathrm{C})$ & 0.9800 \\
\hline $\mathrm{C}(17)-\mathrm{C}(18)$ & $1.511(2)$ \\
\hline $\mathrm{C}(17)-\mathrm{H}(17 \mathrm{~A})$ & 0.9900 \\
\hline
\end{tabular}




$\begin{array}{ll}\mathrm{C}(17)-\mathrm{H}(17 \mathrm{~B}) & 0.9900 \\ \mathrm{C}(18)-\mathrm{C}(19) & 1.394(2) \\ \mathrm{C}(18)-\mathrm{C}(23) & 1.395(2) \\ \mathrm{C}(19)-\mathrm{C}(20) & 1.390(2) \\ \mathrm{C}(19)-\mathrm{H}(19) & 0.9500 \\ \mathrm{C}(20)-\mathrm{C}(21) & 1.386(3) \\ \mathrm{C}(20)-\mathrm{H}(20) & 0.9500 \\ \mathrm{C}(21)-\mathrm{C}(22) & 1.391(3) \\ \mathrm{C}(21)-\mathrm{H}(21) & 0.9500 \\ \mathrm{C}(22)-\mathrm{C}(23) & 1.393(2) \\ \mathrm{C}(22)-\mathrm{H}(22) & 0.9500 \\ \mathrm{C}(23)-\mathrm{H}(23) & 0.9500\end{array}$

Table S9. Bond angles $\left[{ }^{\circ}\right]$ for $\mathbf{2} \bullet[\mathrm{H}][\mathrm{SBn}]$.

\begin{tabular}{lc}
\hline $\mathrm{N}(2)-\mathrm{P}(1)-\mathrm{N}(3)$ & $135.32(6)$ \\
$\mathrm{N}(2)-\mathrm{P}(1)-\mathrm{N}(1)$ & $86.57(6)$ \\
$\mathrm{N}(3)-\mathrm{P}(1)-\mathrm{N}(1)$ & $87.70(6)$ \\
$\mathrm{N}(2)-\mathrm{P}(1)-\mathrm{S}(1)$ & $114.21(5)$ \\
$\mathrm{N}(3)-\mathrm{P}(1)-\mathrm{S}(1)$ & $110.30(5)$ \\
$\mathrm{N}(1)-\mathrm{P}(1)-\mathrm{S}(1)$ & $92.69(4)$ \\
$\mathrm{N}(2)-\mathrm{P}(1)-\mathrm{H}(1)$ & $91.9(10)$ \\
$\mathrm{N}(3)-\mathrm{P}(1)-\mathrm{H}(1)$ & $89.1(10)$ \\
$\mathrm{N}(1)-\mathrm{P}(1)-\mathrm{H}(1)$ & $173.7(10)$ \\
$\mathrm{S}(1)-\mathrm{P}(1)-\mathrm{H}(1)$ & $93.5(10)$ \\
$\mathrm{C}(17)-\mathrm{S}(1)-\mathrm{P}(1)$ & $102.39(5)$ \\
$\mathrm{C}(7)-\mathrm{N}(1)-\mathrm{C}(1)$ & $124.00(11)$ \\
$\mathrm{C}(7)-\mathrm{N}(1)-\mathrm{P}(1)$ & $112.17(9)$ \\
$\mathrm{C}(1)-\mathrm{N}(1)-\mathrm{P}(1)$ & $115.29(10)$ \\
$\mathrm{C}(6)-\mathrm{N}(2)-\mathrm{C}(15)$ & $118.28(12)$ \\
$\mathrm{C}(6)-\mathrm{N}(2)-\mathrm{P}(1)$ & $116.81(10)$ \\
$\mathrm{C}(15)-\mathrm{N}(2)-\mathrm{P}(1)$ & $124.73(10)$ \\
$\mathrm{C}(12)-\mathrm{N}(3)-\mathrm{C}(16)$ & $119.68(12)$ \\
$\mathrm{C}(12)-\mathrm{N}(3)-\mathrm{P}(1)$ & $114.33(9)$ \\
$\mathrm{C}(16)-\mathrm{N}(3)-\mathrm{P}(1)$ & $123.56(10)$ \\
$\mathrm{C}(2)-\mathrm{C}(1)-\mathrm{N}(1)$ & $129.73(13)$ \\
$\mathrm{C}(2)-\mathrm{C}(1)-\mathrm{C}(6)$ & $120.99(13)$ \\
$\mathrm{N}(1)-\mathrm{C}(1)-\mathrm{C}(6)$ & $109.25(12)$ \\
$\mathrm{C}(1)-\mathrm{C}(2)-\mathrm{C}(3)$ & $116.56(14)$ \\
$\mathrm{C}(1)-\mathrm{C}(2)-\mathrm{C}(13)$ & $125.34(13)$ \\
$\mathrm{C}(3)-\mathrm{C}(2)-\mathrm{C}(13)$ & $118.08(13)$ \\
$\mathrm{C}(4)-\mathrm{C}(3)-\mathrm{C}(2)$ & $122.33(14)$ \\
$\mathrm{C}(4)-\mathrm{C}(3)-\mathrm{H}(3)$ & 118.8 \\
$\mathrm{C}(2)-\mathrm{C}(3)-\mathrm{H}(3)$ & 118.8 \\
$\mathrm{C}(3)-\mathrm{C}(4)-\mathrm{C}(5)$ & $120.55(14)$ \\
$\mathrm{C}(3)-\mathrm{C}(4)-\mathrm{H}(4)$ & 119.7 \\
$\mathrm{C}(5)-\mathrm{C}(4)-\mathrm{H}(4)$ & 119.7 \\
$\mathrm{C}(6)-\mathrm{C}(5)-\mathrm{C}(4)$ & $117.91(14)$ \\
& \\
&
\end{tabular}




\begin{tabular}{|c|c|}
\hline $\mathrm{C}(6)-\mathrm{C}(5)-\mathrm{H}(5)$ & 121.0 \\
\hline $\mathrm{C}(4)-\mathrm{C}(5)-\mathrm{H}(5)$ & 121.0 \\
\hline$C(5)-C(6)-N(2)$ & $126.32(13)$ \\
\hline$C(5)-C(6)-C(1)$ & $121.58(13)$ \\
\hline$N(2)-C(6)-C(1)$ & $112.03(12)$ \\
\hline $\mathrm{C}(8)-\mathrm{C}(7)-\mathrm{N}(1)$ & $126.16(12)$ \\
\hline $\mathrm{C}(8)-\mathrm{C}(7)-\mathrm{C}(12)$ & $122.79(12)$ \\
\hline $\mathrm{N}(1)-\mathrm{C}(7)-\mathrm{C}(12)$ & $111.04(12)$ \\
\hline $\mathrm{C}(7)-\mathrm{C}(8)-\mathrm{C}(9)$ & $116.89(13)$ \\
\hline$C(7)-C(8)-C(14)$ & $119.63(12)$ \\
\hline $\mathrm{C}(9)-\mathrm{C}(8)-\mathrm{C}(14)$ & $123.47(13)$ \\
\hline $\mathrm{C}(10)-\mathrm{C}(9)-\mathrm{C}(8)$ & $120.98(14)$ \\
\hline $\mathrm{C}(10)-\mathrm{C}(9)-\mathrm{H}(9)$ & 119.5 \\
\hline $\mathrm{C}(8)-\mathrm{C}(9)-\mathrm{H}(9)$ & 119.5 \\
\hline$C(9)-C(10)-C(11)$ & $121.49(14)$ \\
\hline $\mathrm{C}(9)-\mathrm{C}(10)-\mathrm{H}(10)$ & 119.3 \\
\hline $\mathrm{C}(11)-\mathrm{C}(10)-\mathrm{H}(10)$ & 119.3 \\
\hline $\mathrm{C}(12)-\mathrm{C}(11)-\mathrm{C}(10)$ & $117.96(14)$ \\
\hline $\mathrm{C}(12)-\mathrm{C}(11)-\mathrm{H}(11)$ & 121.0 \\
\hline $\mathrm{C}(10)-\mathrm{C}(11)-\mathrm{H}(11)$ & 121.0 \\
\hline $\mathrm{C}(11)-\mathrm{C}(12)-\mathrm{N}(3)$ & $128.35(13)$ \\
\hline$C(11)-C(12)-C(7)$ & $119.89(13)$ \\
\hline $\mathrm{N}(3)-\mathrm{C}(12)-\mathrm{C}(7)$ & $111.75(12)$ \\
\hline $\mathrm{C}(2)-\mathrm{C}(13)-\mathrm{C}(14)$ & $115.98(11)$ \\
\hline $\mathrm{C}(2)-\mathrm{C}(13)-\mathrm{H}(13 \mathrm{~A})$ & 108.3 \\
\hline $\mathrm{C}(14)-\mathrm{C}(13)-\mathrm{H}(13 \mathrm{~A})$ & 108.3 \\
\hline $\mathrm{C}(2)-\mathrm{C}(13)-\mathrm{H}(13 \mathrm{~B})$ & 108.3 \\
\hline $\mathrm{C}(14)-\mathrm{C}(13)-\mathrm{H}(13 \mathrm{~B})$ & 108.3 \\
\hline $\mathrm{H}(13 \mathrm{~A})-\mathrm{C}(13)-\mathrm{H}(13 \mathrm{~B})$ & 107.4 \\
\hline $\mathrm{C}(8)-\mathrm{C}(14)-\mathrm{C}(13)$ & $113.64(12)$ \\
\hline $\mathrm{C}(8)-\mathrm{C}(14)-\mathrm{H}(14 \mathrm{~A})$ & 108.8 \\
\hline $\mathrm{C}(13)-\mathrm{C}(14)-\mathrm{H}(14 \mathrm{~A})$ & 108.8 \\
\hline $\mathrm{C}(8)-\mathrm{C}(14)-\mathrm{H}(14 \mathrm{~B})$ & 108.8 \\
\hline $\mathrm{C}(13)-\mathrm{C}(14)-\mathrm{H}(14 \mathrm{~B})$ & 108.8 \\
\hline $\mathrm{H}(14 \mathrm{~A})-\mathrm{C}(14)-\mathrm{H}(14 \mathrm{~B})$ & 107.7 \\
\hline $\mathrm{N}(2)-\mathrm{C}(15)-\mathrm{H}(15 \mathrm{~A})$ & 109.5 \\
\hline $\mathrm{N}(2)-\mathrm{C}(15)-\mathrm{H}(15 \mathrm{~B})$ & 109.5 \\
\hline $\mathrm{H}(15 \mathrm{~A})-\mathrm{C}(15)-\mathrm{H}(15 \mathrm{~B})$ & 109.5 \\
\hline $\mathrm{N}(2)-\mathrm{C}(15)-\mathrm{H}(15 \mathrm{C})$ & 109.5 \\
\hline $\mathrm{H}(15 \mathrm{~A})-\mathrm{C}(15)-\mathrm{H}(15 \mathrm{C})$ & 109.5 \\
\hline $\mathrm{H}(15 \mathrm{~B})-\mathrm{C}(15)-\mathrm{H}(15 \mathrm{C})$ & 109.5 \\
\hline $\mathrm{N}(3)-\mathrm{C}(16)-\mathrm{H}(16 \mathrm{~A})$ & 109.5 \\
\hline $\mathrm{N}(3)-\mathrm{C}(16)-\mathrm{H}(16 \mathrm{~B})$ & 109.5 \\
\hline $\mathrm{H}(16 \mathrm{~A})-\mathrm{C}(16)-\mathrm{H}(16 \mathrm{~B})$ & 109.5 \\
\hline $\mathrm{N}(3)-\mathrm{C}(16)-\mathrm{H}(16 \mathrm{C})$ & 109.5 \\
\hline $\mathrm{H}(16 \mathrm{~A})-\mathrm{C}(16)-\mathrm{H}(16 \mathrm{C})$ & 109.5 \\
\hline $\mathrm{H}(16 \mathrm{~B})-\mathrm{C}(16)-\mathrm{H}(16 \mathrm{C})$ & 109.5 \\
\hline $\mathrm{C}(18)-\mathrm{C}(17)-\mathrm{S}(1)$ & $109.07(10)$ \\
\hline $\mathrm{C}(18)-\mathrm{C}(17)-\mathrm{H}(17 \mathrm{~A})$ & 109.9 \\
\hline $\mathrm{S}(1)-\mathrm{C}(17)-\mathrm{H}(17 \mathrm{~A})$ & 109.9 \\
\hline $\mathrm{C}(18)-\mathrm{C}(17)-\mathrm{H}(17 \mathrm{~B})$ & 109.9 \\
\hline
\end{tabular}




$\begin{array}{ll}\mathrm{S}(1)-\mathrm{C}(17)-\mathrm{H}(17 \mathrm{~B}) & 109.9 \\ \mathrm{H}(17 \mathrm{~A})-\mathrm{C}(17)-\mathrm{H}(17 \mathrm{~B}) & 108.3 \\ \mathrm{C}(19)-\mathrm{C}(18)-\mathrm{C}(23) & 118.68(13) \\ \mathrm{C}(19)-\mathrm{C}(18)-\mathrm{C}(17) & 120.04(14) \\ \mathrm{C}(23)-\mathrm{C}(18)-\mathrm{C}(17) & 121.28(13) \\ \mathrm{C}(20)-\mathrm{C}(19)-\mathrm{C}(18) & 120.97(15) \\ \mathrm{C}(20)-\mathrm{C}(19)-\mathrm{H}(19) & 119.5 \\ \mathrm{C}(18)-\mathrm{C}(19)-\mathrm{H}(19) & 119.5 \\ \mathrm{C}(21)-\mathrm{C}(20)-\mathrm{C}(19) & 120.00(16) \\ \mathrm{C}(21)-\mathrm{C}(20)-\mathrm{H}(20) & 120.0 \\ \mathrm{C}(19)-\mathrm{C}(20)-\mathrm{H}(20) & 120.0 \\ \mathrm{C}(20)-\mathrm{C}(21)-\mathrm{C}(22) & 119.67(16) \\ \mathrm{C}(20)-\mathrm{C}(21)-\mathrm{H}(21) & 120.2 \\ \mathrm{C}(22)-\mathrm{C}(21)-\mathrm{H}(21) & 120.2 \\ \mathrm{C}(21)-\mathrm{C}(22)-\mathrm{C}(23) & 120.25(17) \\ \mathrm{C}(21)-\mathrm{C}(22)-\mathrm{H}(22) & 119.9 \\ \mathrm{C}(23)-\mathrm{C}(22)-\mathrm{H}(22) & 119.9 \\ \mathrm{C}(22)-\mathrm{C}(23)-\mathrm{C}(18) & 120.43(15) \\ \mathrm{C}(22)-\mathrm{C}(23)-\mathrm{H}(23) & 119.8 \\ \mathrm{C}(18)-\mathrm{C}(23)-\mathrm{H}(23) & 119.8\end{array}$




\section{Computational Details}

\section{A. General computational information}

Geometries were optimized in Orca 4.0.0. ${ }^{5}$ Calculations were carried out in the gas phase without symmetry constraint. Stationary points were characterized by frequency calculations to confirm their identity as local minima (zero imaginary frequencies).

B. Absolute thermochemical data $\left(\omega \mathrm{B} 97 \mathrm{X}-\mathrm{D} 3^{6} / 6-311++\mathrm{G}(2 \mathrm{~d}, 2 \mathrm{p})^{7}\right.$, in Hartrees)

$\mathrm{MeSH}$

Temperature $=298.150 \mathrm{~K}$

Pressure $=1.00000$ Atm

Electronic Energy $=-438.71247004$

Total Thermal Energy $=-438.66240390$

Enthalpy $=-438.66145969$

Free Energy $=-438.68912003$

\section{1}

Temperature $=298.150 \mathrm{~K}$

Pressure $=1.00000$ Atm

Electronic Energy = -1047.54660658

Total Thermal Energy $=-1047.27340715$

Enthalpy $=-1047.27246294$

Free Energy = -1047.32785174

$\mathbf{1} \cdot[\mathrm{H}][\mathrm{SMe}]$

Temperature $=298.150 \mathrm{~K}$

Pressure $=1.00000$ Atm

Electronic Energy = -1486.28400213

Total Thermal Energy $=-1485.95566763$

Enthalpy $=-1485.95472342$

Free Energy = -1486.01752772

7•[SMe $]$

Temperature $=298.150 \mathrm{~K}$

Pressure $=1.00000$ Atm

Electronic Energy = -1486.29238869

Total Thermal Energy $=-1485.96298897$

Enthalpy $=-1485.96204476$

Free Energy = -1486.02801682

2

Temperature $=298.150 \mathrm{~K}$

Pressure $=1.00000$ Atm

Electronic Energy = -1124.98160036

Total Thermal Energy $=-1124.67081147$ 
Enthalpy $=-1124.66986726$

Free Energy = -1124.72700196

$\mathbf{2} \cdot[\mathrm{H}][\mathrm{SMe}]$

Temperature $=298.150 \mathrm{~K}$

Pressure $=1.00000$ Atm

Electronic Energy = -1563.72008551

Total Thermal Energy $=-1563.35390151$

Enthalpy $=-1563.35295731$

Free Energy = -1563.41724239

$\mathbf{8} \cdot[\mathrm{SMe}]$

Temperature $=298.150 \mathrm{~K}$

Pressure $=1.00000$ Atm

Electronic Energy = -1563.70572446

Total Thermal Energy $=-1563.33859890$

Enthalpy $=-1563.33765469$

Free Energy $=-1563.40356638$

C. Natural Bond Orbital (NBO) analysis and frontier molecular orbitals (B3LYP ${ }^{8} / D^{2} 2-T Z V P^{9}$ )

1

26. (1.96268) LP ( 1) P $32 \quad s(61.66 \%)$ p $0.62(38.29 \%) d 0.00(0.05 \%) f 0.00(0.00 \%)$

$0.00000 .00000 .7850-0.02090 .0001$

$0.0000-0.0716-0.0048-0.00080 .0003$

$0.0000-0.5740 \quad 0.0356-0.00180 .0002$

$\begin{array}{lllll}0.0000 & -0.2164 & 0.0138 & 0.0008 & 0.0000\end{array}$

$\begin{array}{lllll}0.0005 & -0.0012-0.0011 & 0.0001 & 0.0056\end{array}$

$\begin{array}{llll}-0.0022 & 0.0119 & 0.0055 & -0.0165-0.0008\end{array}$

$0.00020 .00160 .0006-0.0006-0.0003$

0.00150 .0023

2

25. (1.96105) LP ( 1) P 1 s ( 62.33\%) p 0.60(37.60\%) d 0.00( $0.06 \%)$ f $0.00(0.00 \%)$

$0.00000 .0000 \quad 0.7892-0.02090 .0000$

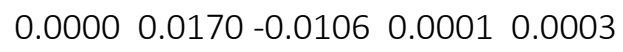

$0.0000-0.6053 \quad 0.0365-0.00210 .0001$

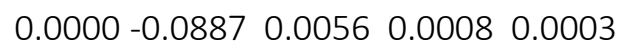

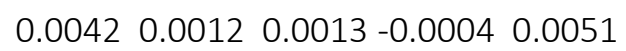

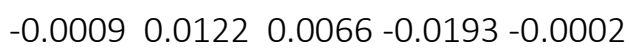

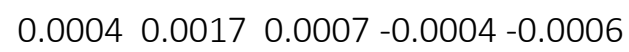

0.00040 .0027 
Table S10. Frontier molecular orbitals and energies.

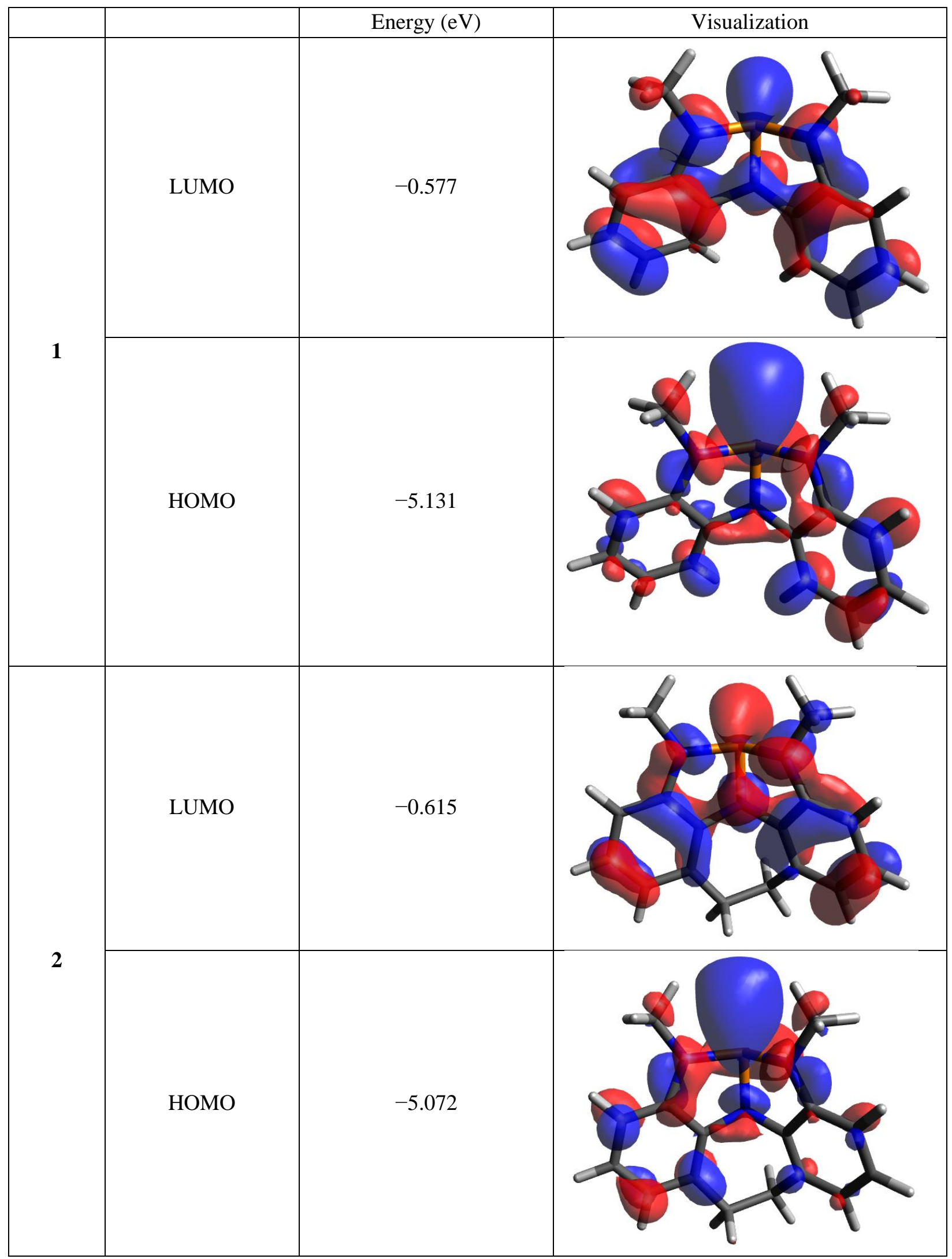


VII. Multinuclear NMR Spectra

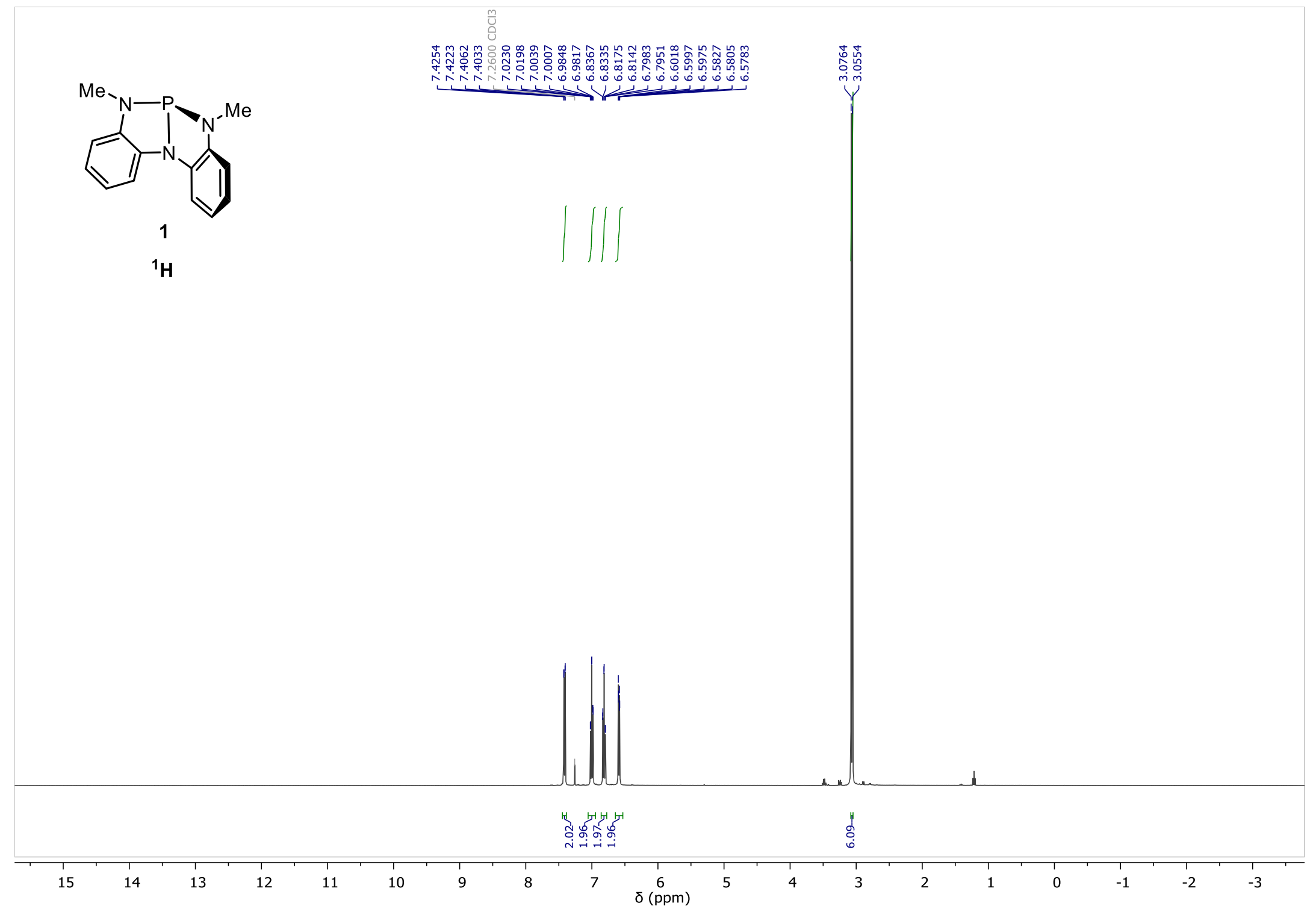

Figure S13. ${ }^{1} \mathrm{H}$ NMR spectrum of 1 recorded in $\mathrm{CDCl}_{3}$ at $23{ }^{\circ} \mathrm{C}$. 


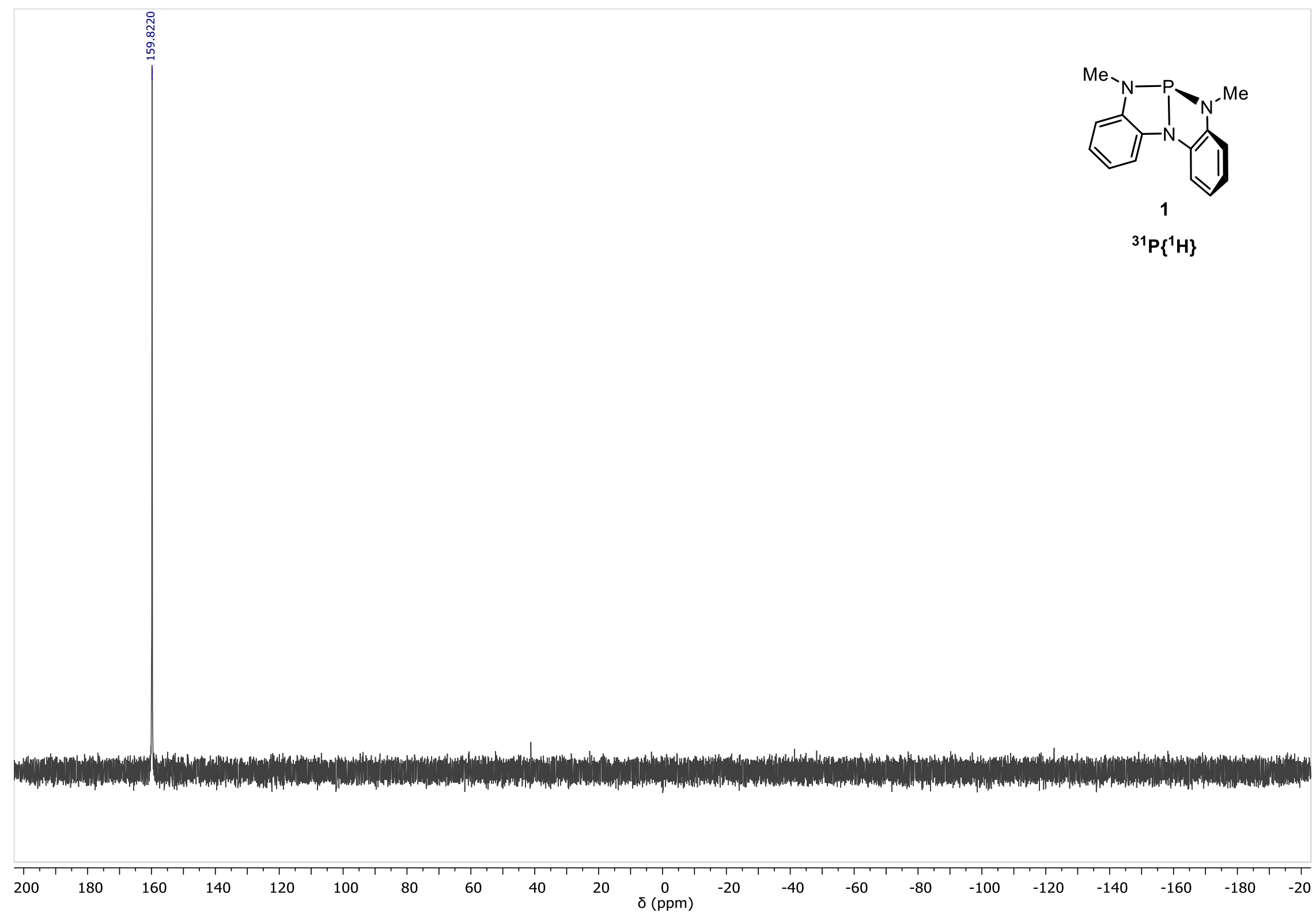

Figure S14. ${ }^{31} \mathrm{P}\left\{{ }^{1} \mathrm{H}\right\}$ NMR spectrum of 1 recorded in $\mathrm{CDCl}_{3}$ at $23{ }^{\circ} \mathrm{C}$. 


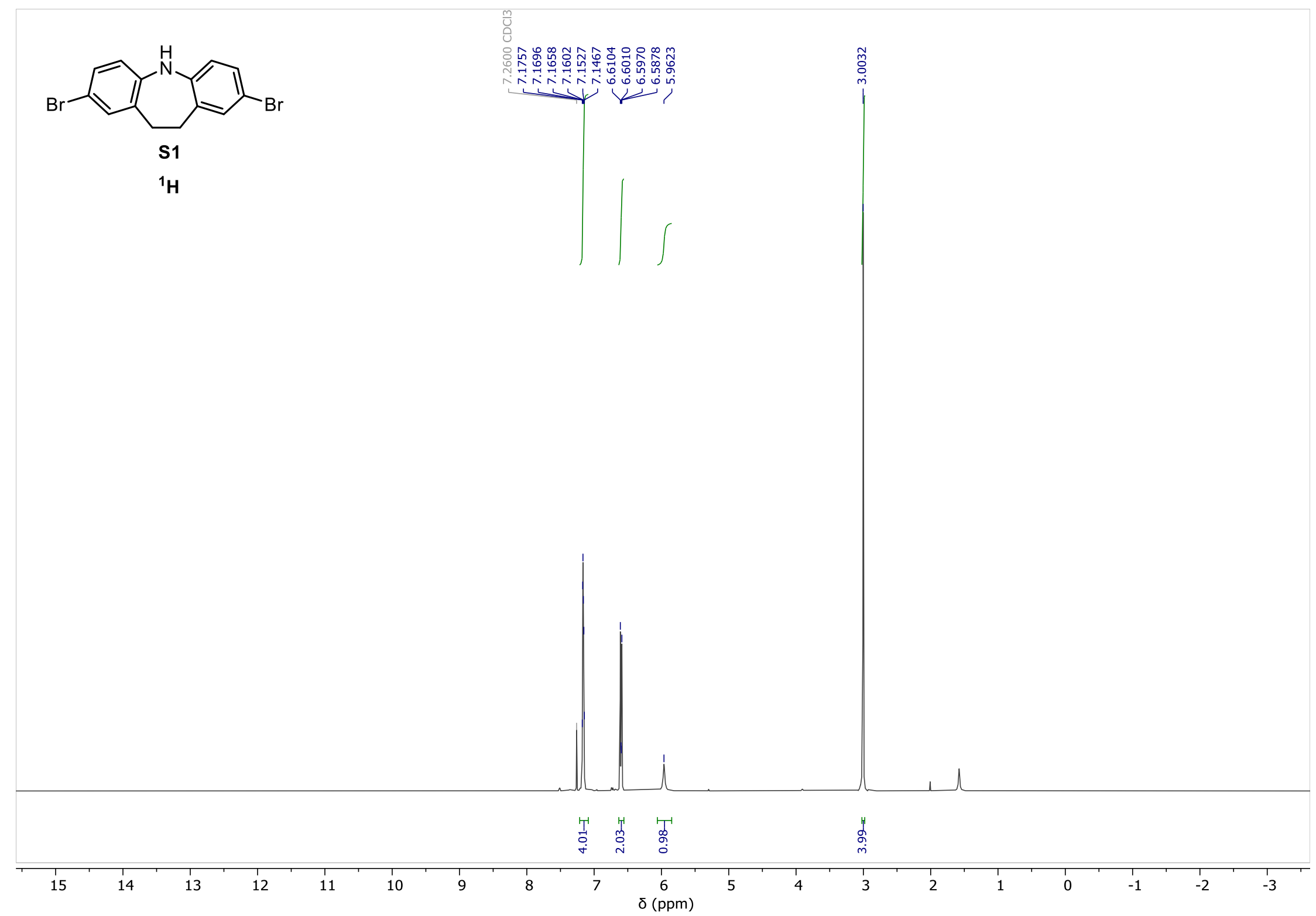

Figure S15. ${ }^{1} \mathrm{H}$ NMR spectrum of $\mathbf{S} 1$ recorded in $\mathrm{CDCl}_{3}$ at $23{ }^{\circ} \mathrm{C}$. 


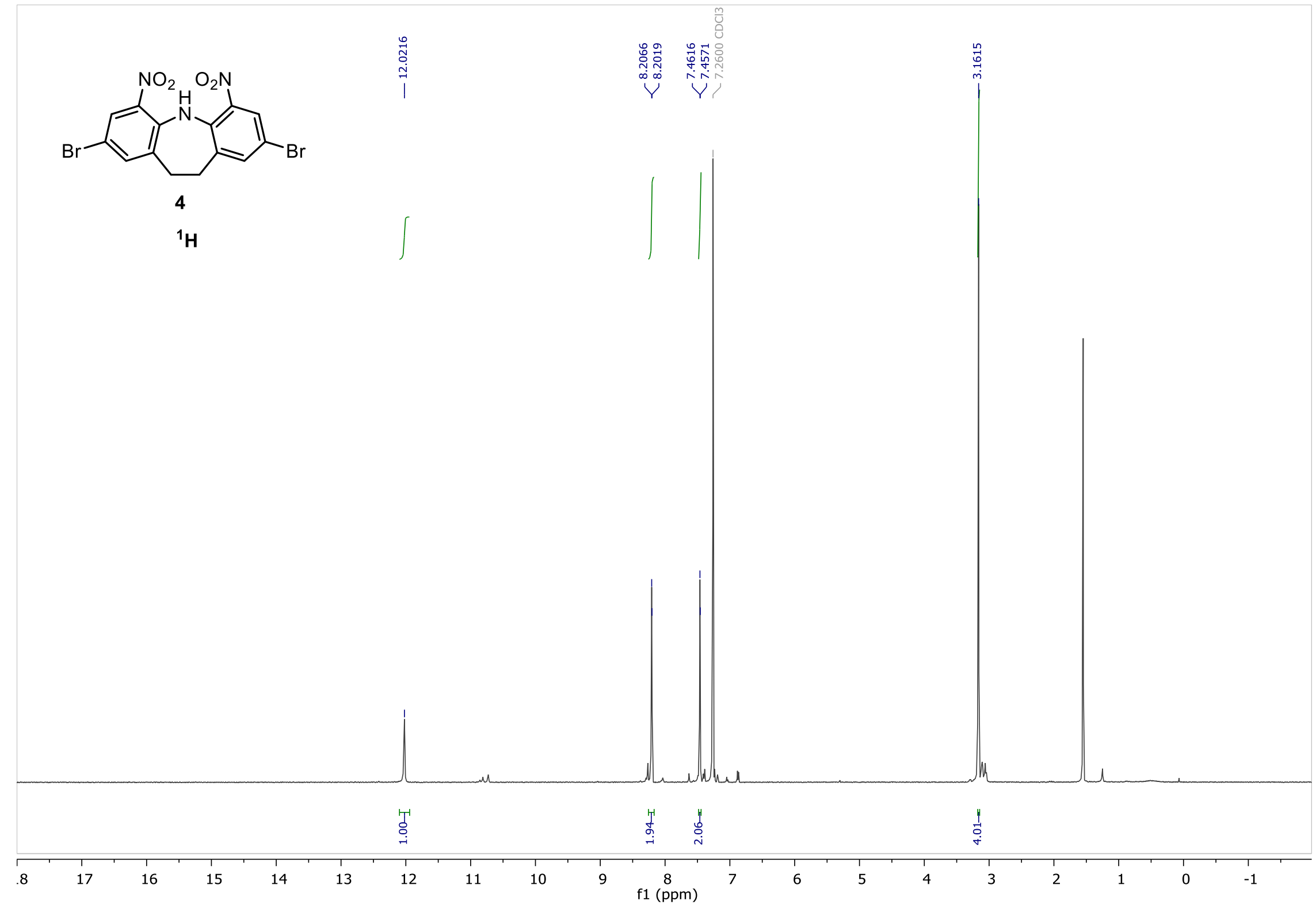

Figure S16. ${ }^{1} \mathrm{H}$ NMR spectrum of 4 recorded in $\mathrm{CDCl}_{3}$ at $23{ }^{\circ} \mathrm{C}$. 


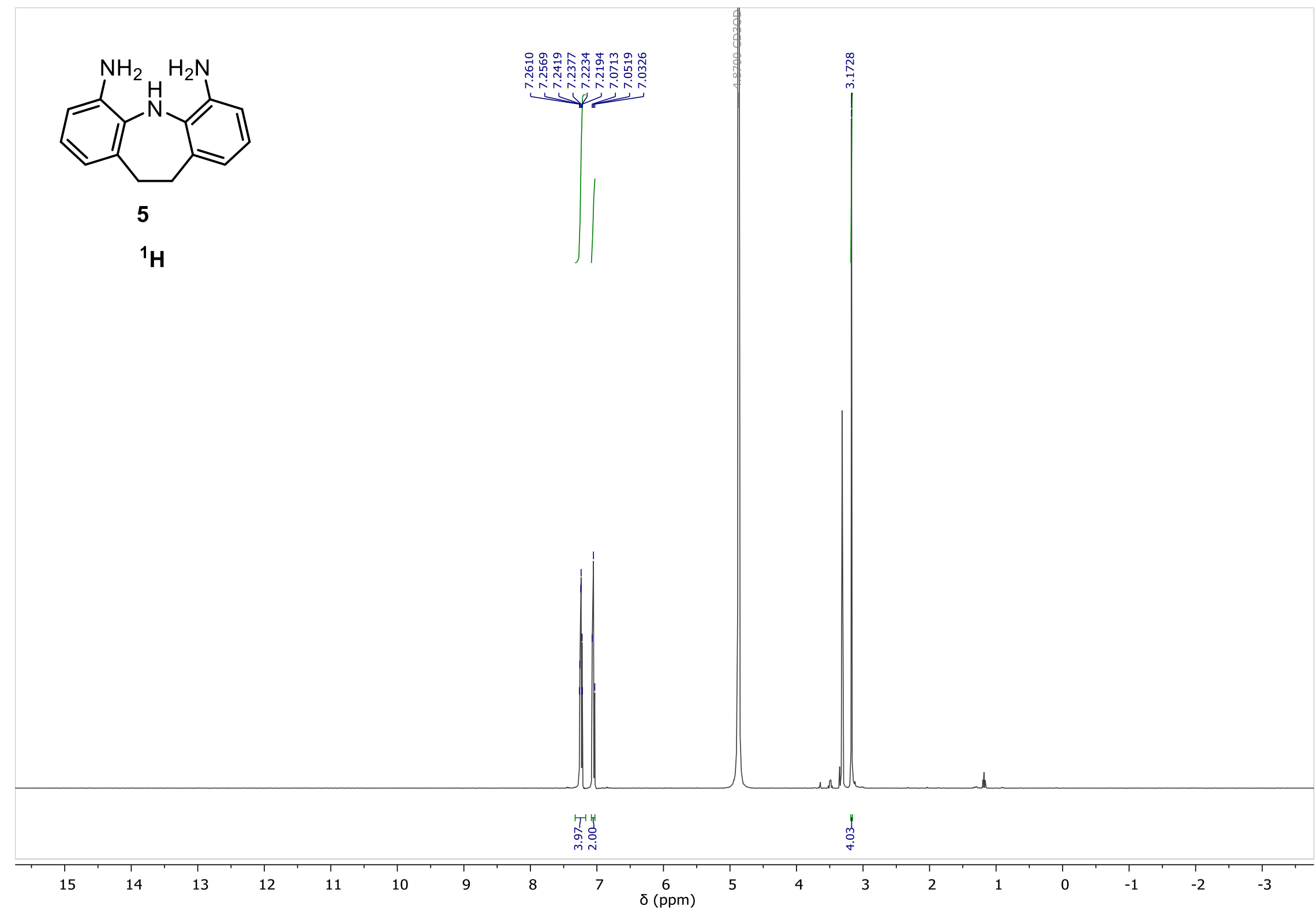

Figure S17. ${ }^{1} \mathrm{H}$ NMR spectrum of 5 recorded in $\mathrm{CD}_{3} \mathrm{OD}$ at $23{ }^{\circ} \mathrm{C}$. 


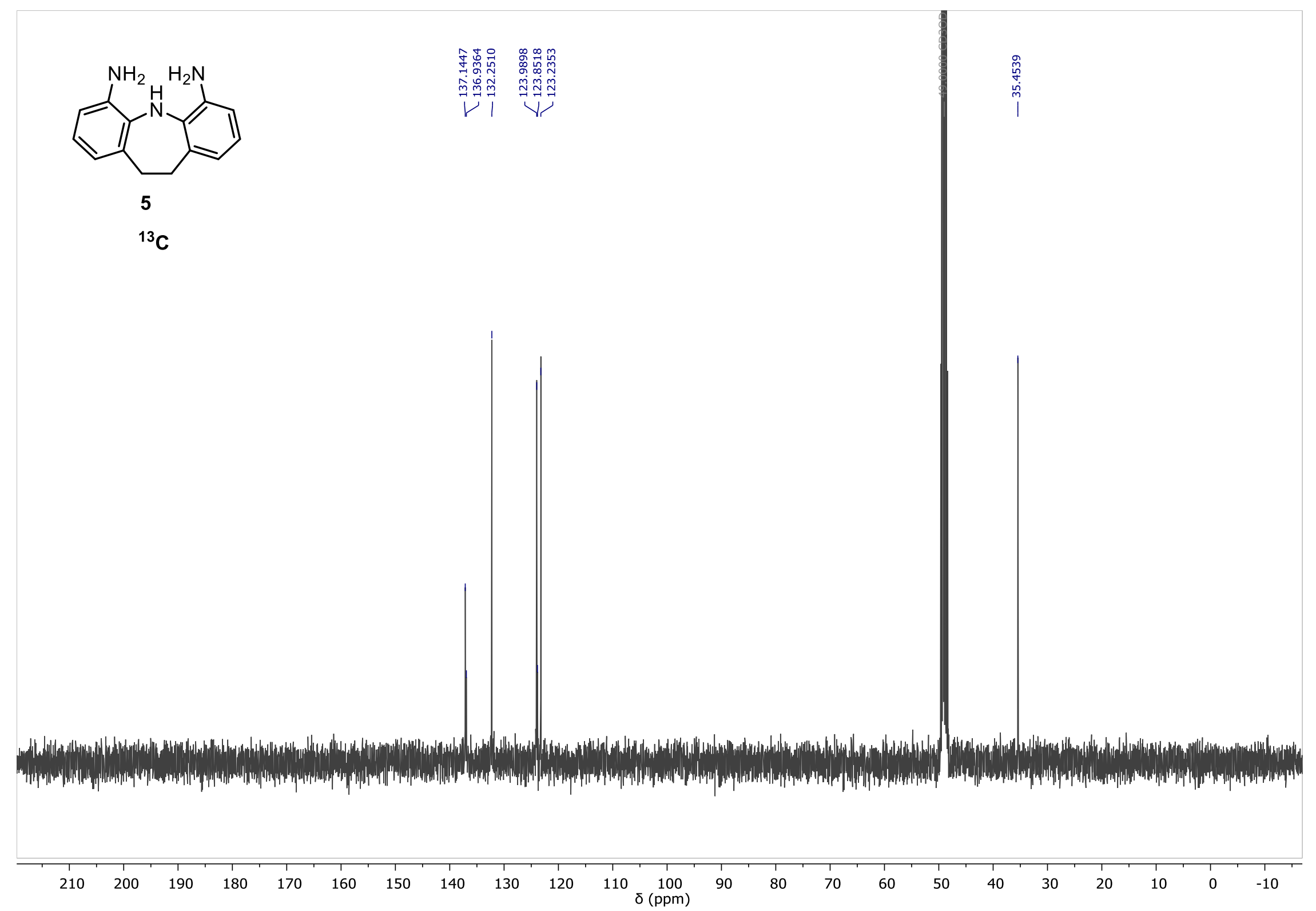

Figure S18. ${ }^{13} \mathrm{C}$ NMR spectrum of 5 recorded in $\mathrm{CD}_{3} \mathrm{OD}$ at $23{ }^{\circ} \mathrm{C}$. 


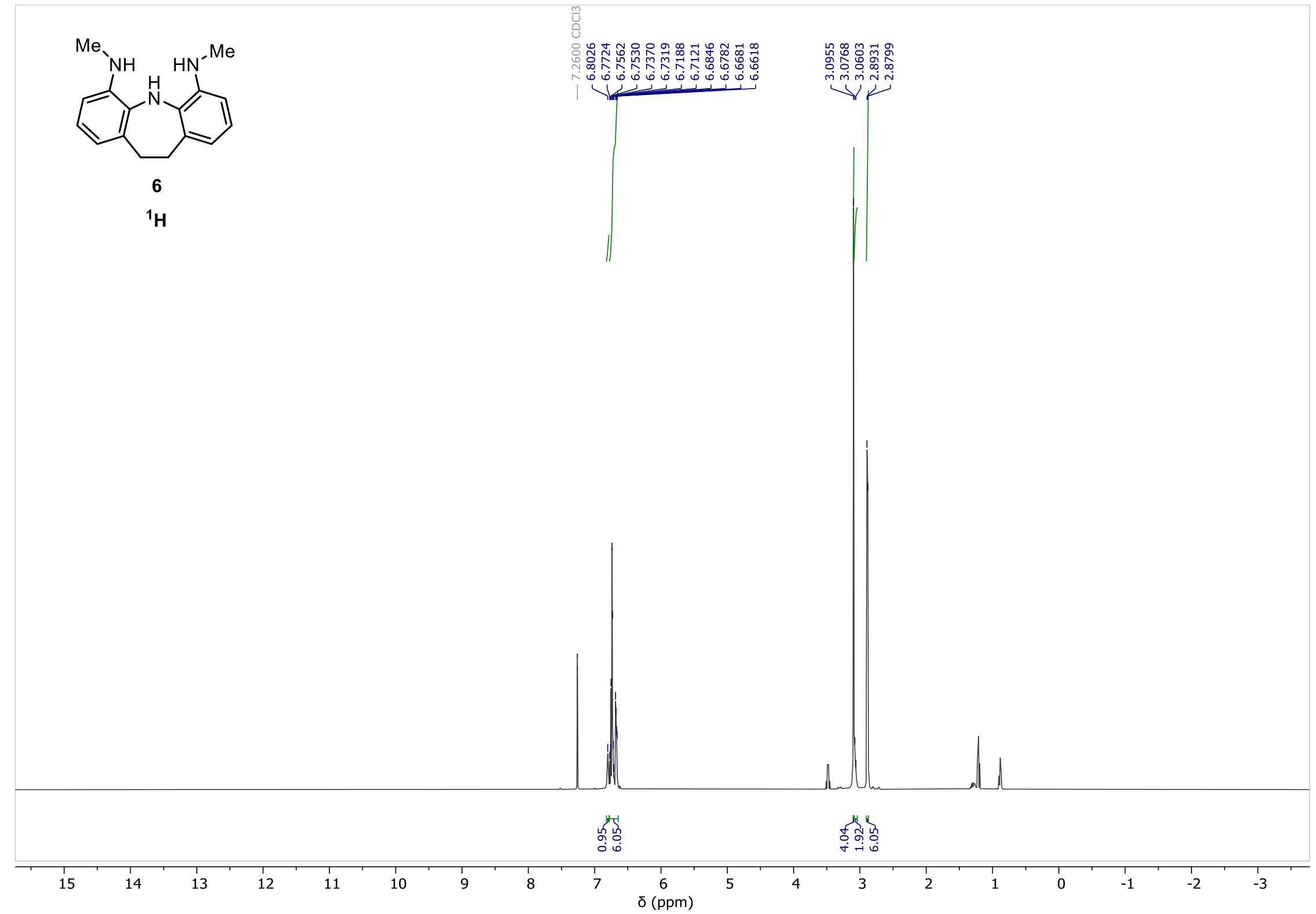

Figure S19. ${ }^{1} \mathrm{H}$ NMR spectrum of 6 recorded in $\mathrm{CDCl}_{3}$ at $23{ }^{\circ} \mathrm{C}$. 


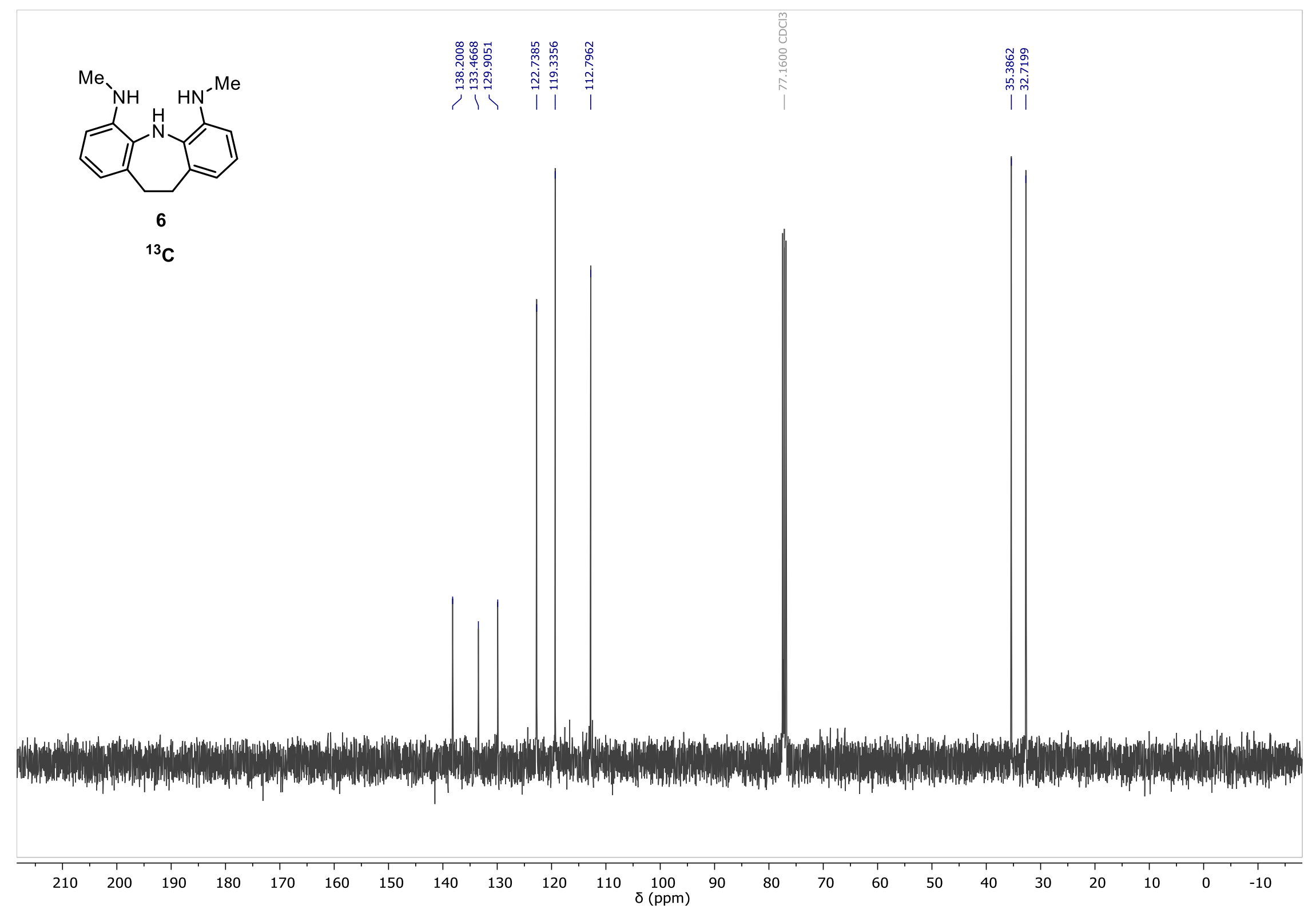

Figure S20. ${ }^{13} \mathrm{C}$ NMR spectrum of 6 recorded in $\mathrm{CDCl}_{3}$ at $23{ }^{\circ} \mathrm{C}$. 


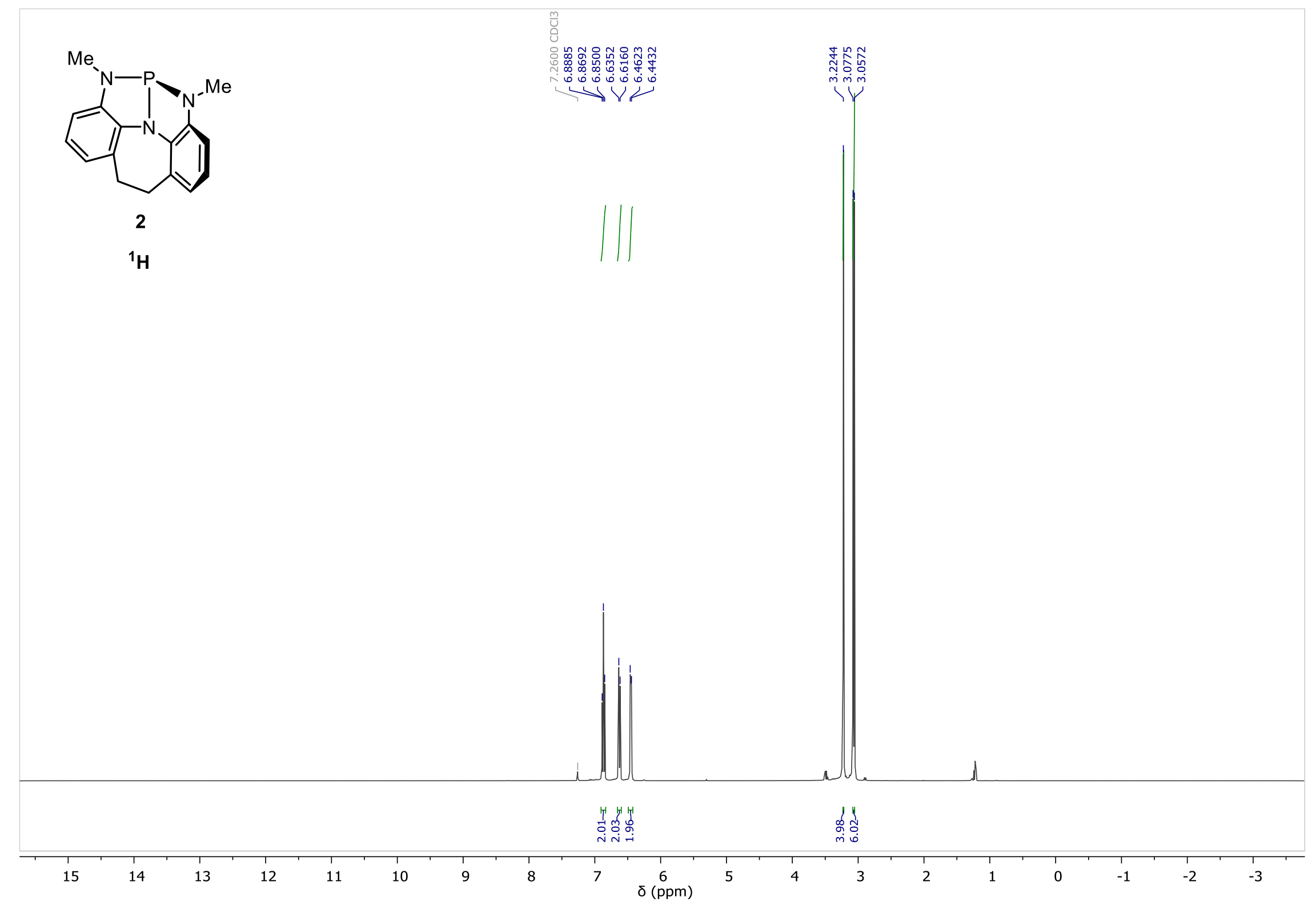

Figure S21. ${ }^{1} \mathrm{H}$ NMR spectrum of 2 recorded in $\mathrm{CDCl}_{3}$ at $23{ }^{\circ} \mathrm{C}$. 


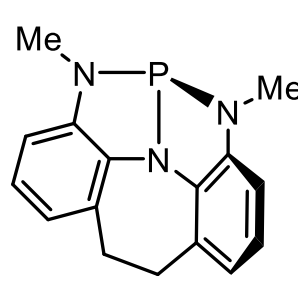

2

${ }^{13} \mathrm{C}$

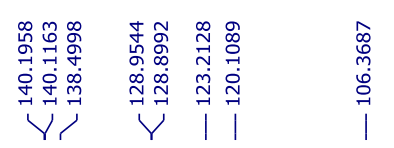

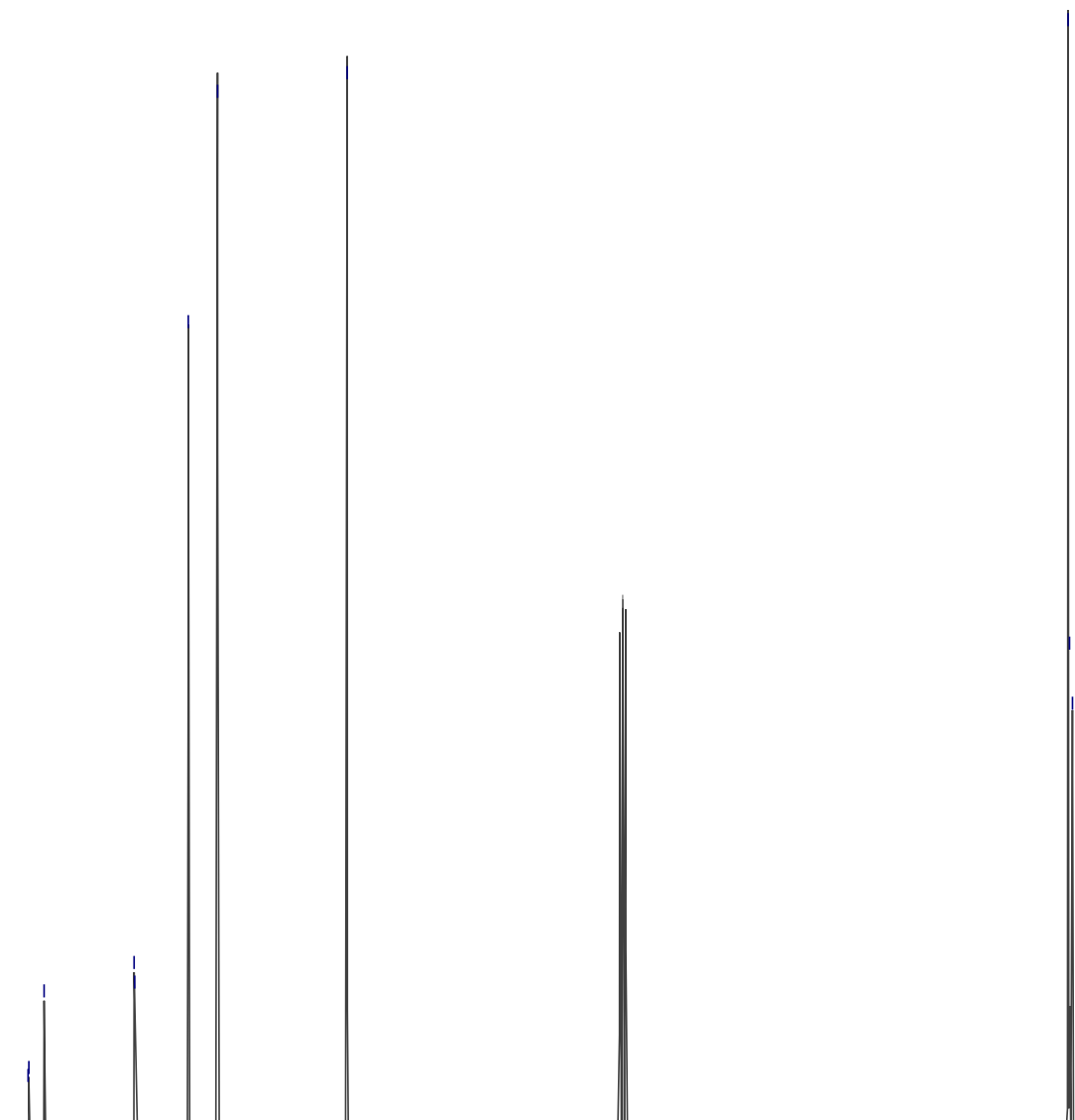

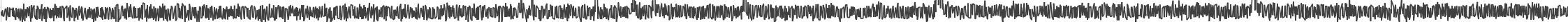

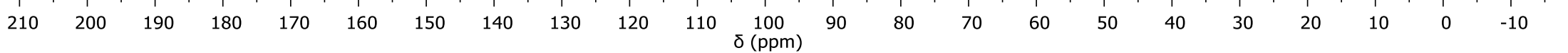

Figure S22. ${ }^{13} \mathrm{C}$ NMR spectrum of 2 recorded in $\mathrm{CDCl}_{3}$ at $23{ }^{\circ} \mathrm{C}$. 


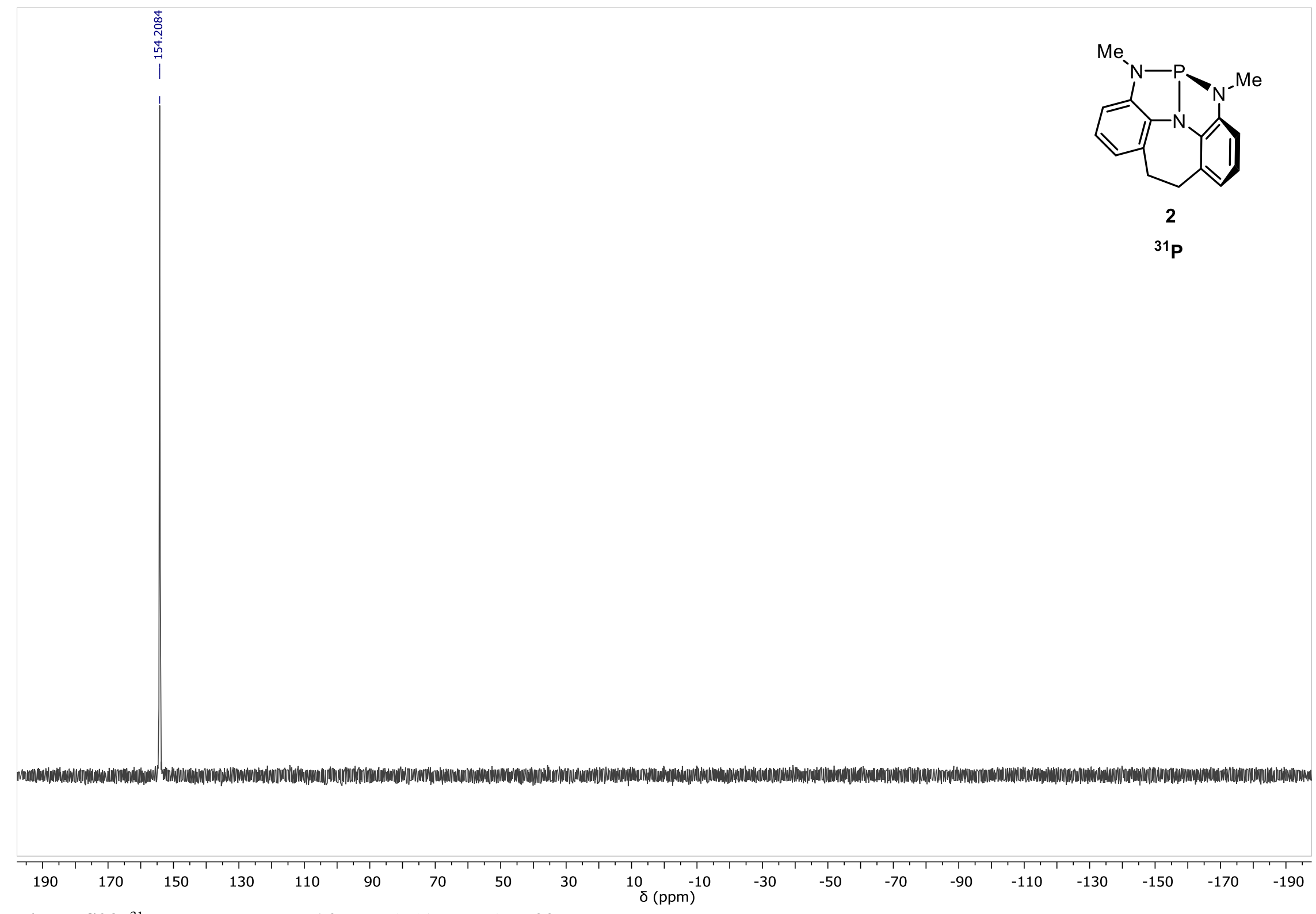

Figure S23. ${ }^{31} \mathrm{P}$ NMR spectrum of 2 recorded in $\mathrm{CDCl}_{3}$ at $23{ }^{\circ} \mathrm{C}$. 


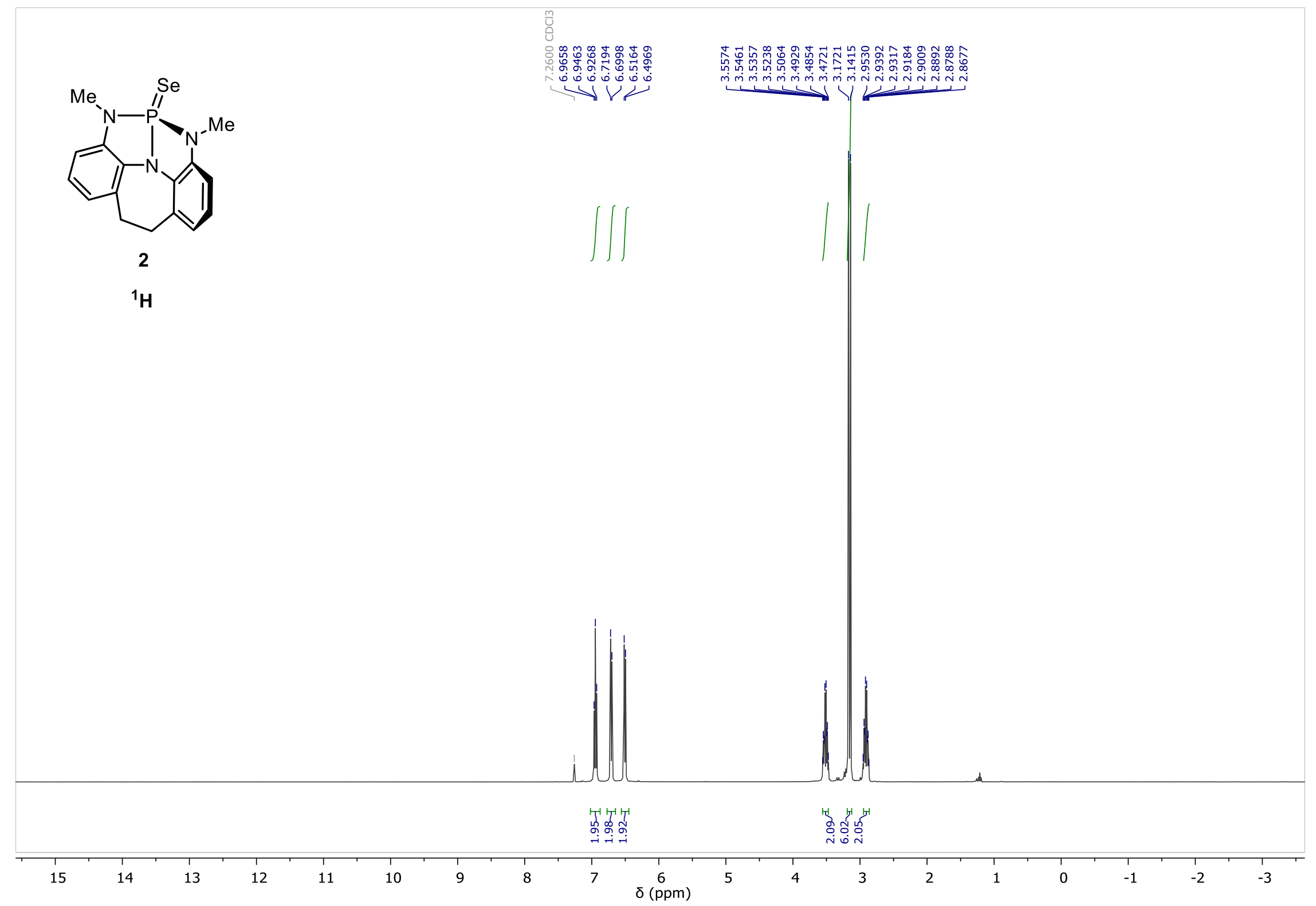

Figure S24. ${ }^{1} \mathrm{H}$ NMR spectrum of $\mathbf{2} \cdot[\mathrm{Se}]$ recorded in $\mathrm{CDCl}_{3}$ at $23{ }^{\circ} \mathrm{C}$. 


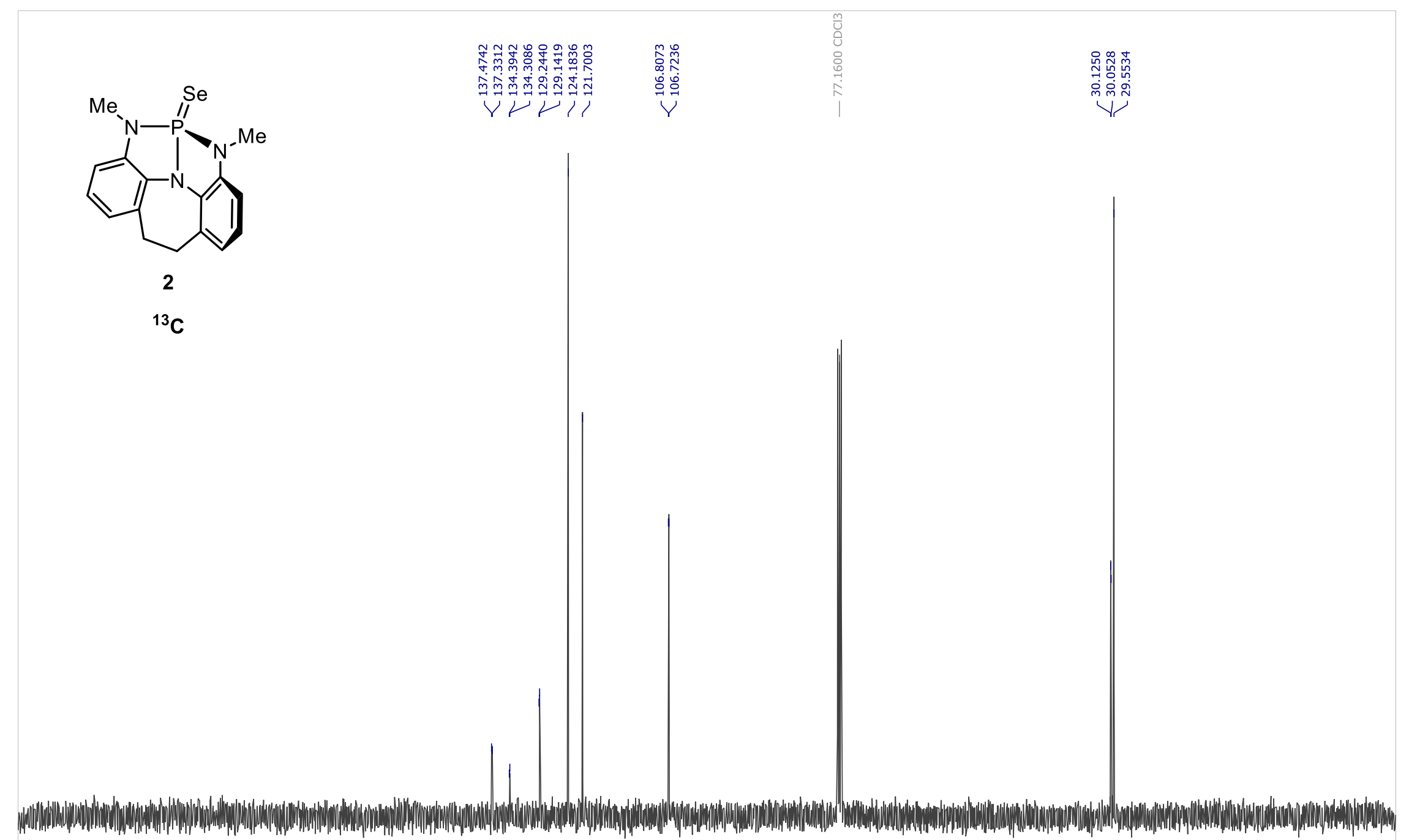

$\begin{array}{lllllllll}210 & 200 & 190 & 180 & 170 & 160 & 150 & 140 & 130\end{array}$ 110 $\delta(\mathrm{ppm})$ $90 \quad 80 \quad 70$ 60 50 40 $30 \quad 20$ 10 $0+10$

Figure $\mathbf{S 2 5} .{ }^{13} \mathrm{C}$ NMR spectrum of $2 \cdot[\mathrm{Se}]$ recorded in $\mathrm{CDCl}_{3}$ at $23{ }^{\circ} \mathrm{C}$. 


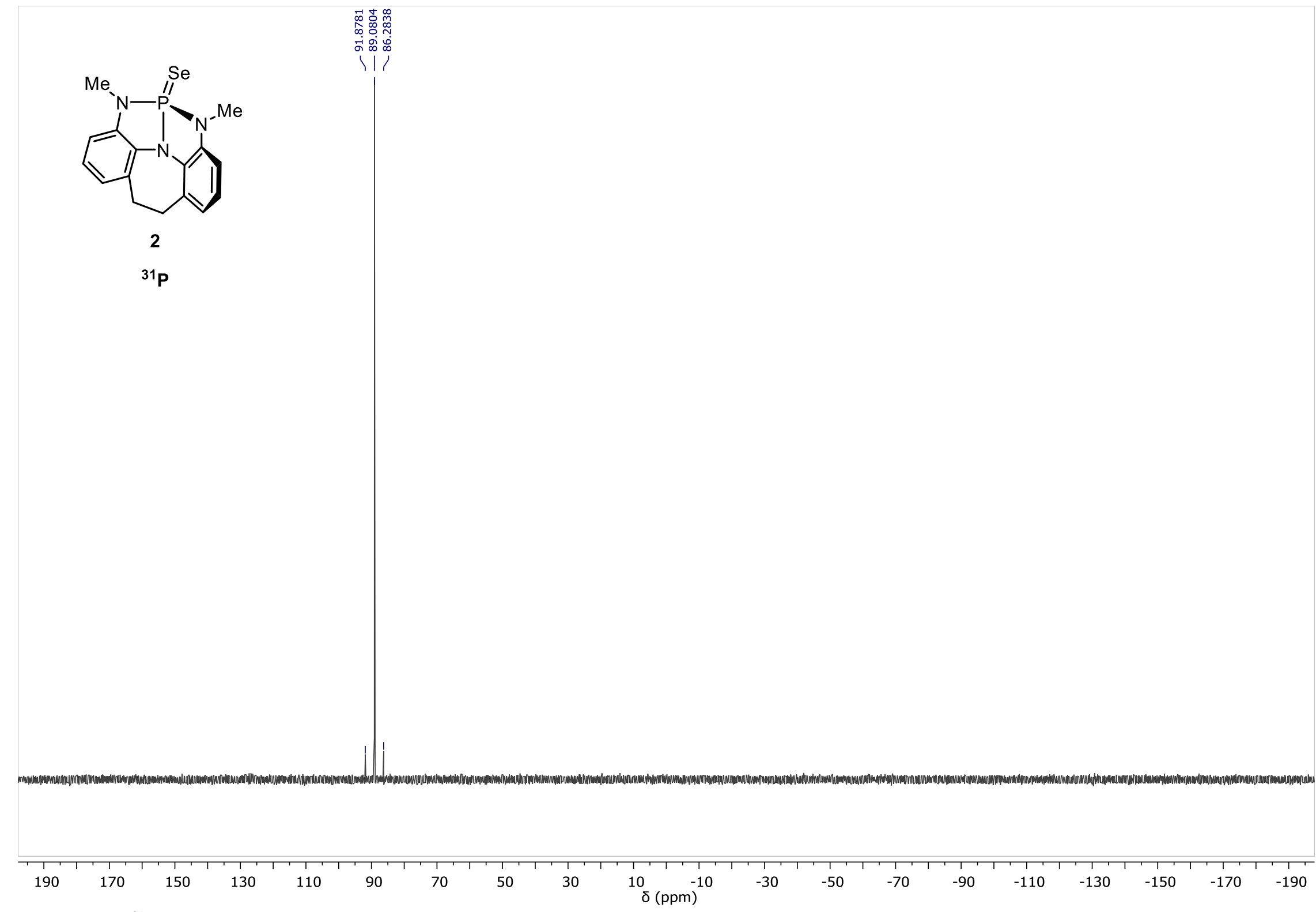

Figure S26. ${ }^{31} \mathrm{P}$ NMR spectrum of $\mathbf{2} \cdot[\mathrm{Se}]$ recorded in $\mathrm{CDCl}_{3}$ at $23{ }^{\circ} \mathrm{C}$. 


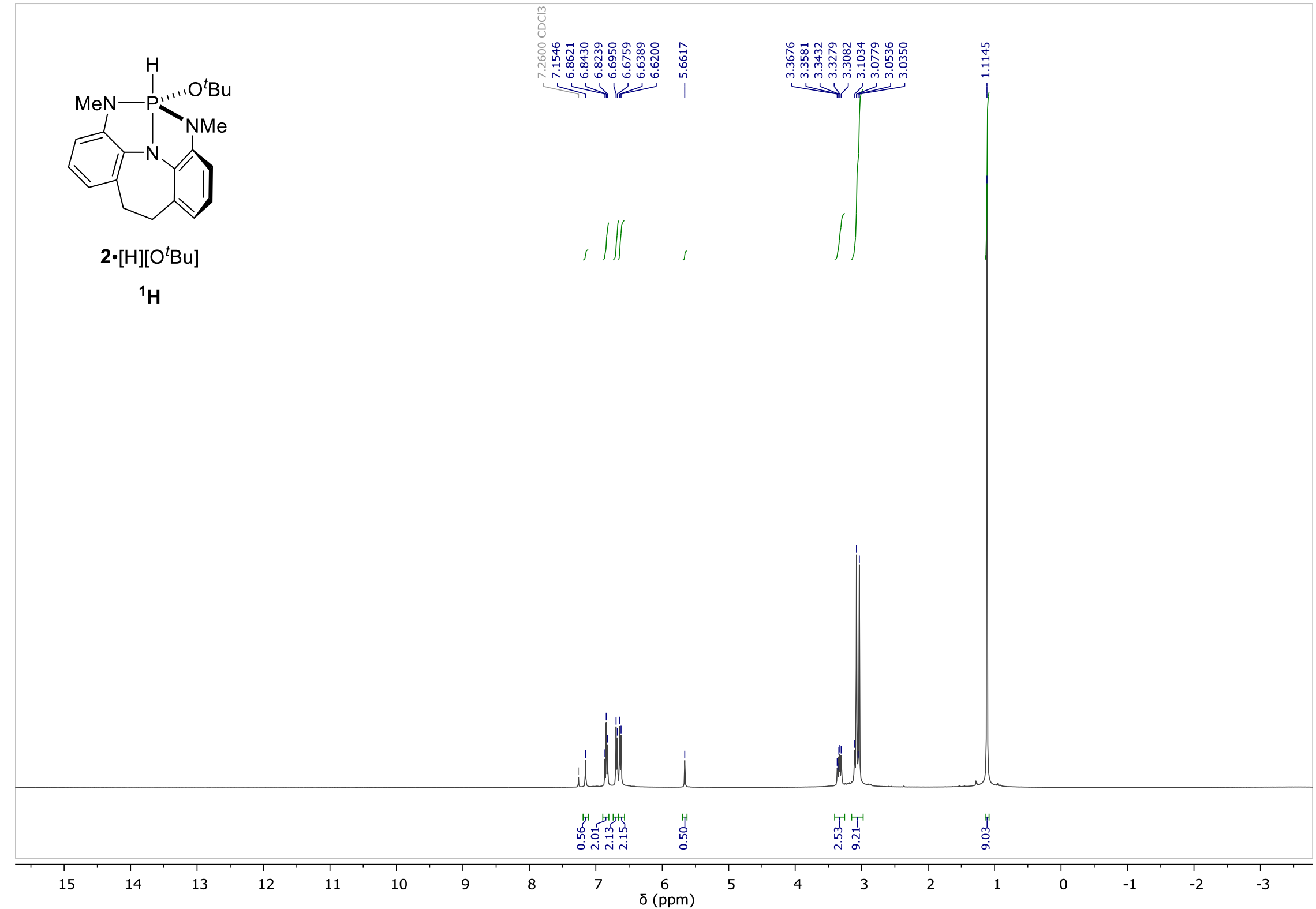

Figure S27. ${ }^{1} \mathrm{H}$ NMR spectrum of $\mathbf{2} \cdot[\mathrm{H}][\mathrm{O}-t-\mathrm{Bu}]$ recorded in $\mathrm{CDCl}_{3}$ at $23{ }^{\circ} \mathrm{C}$. 


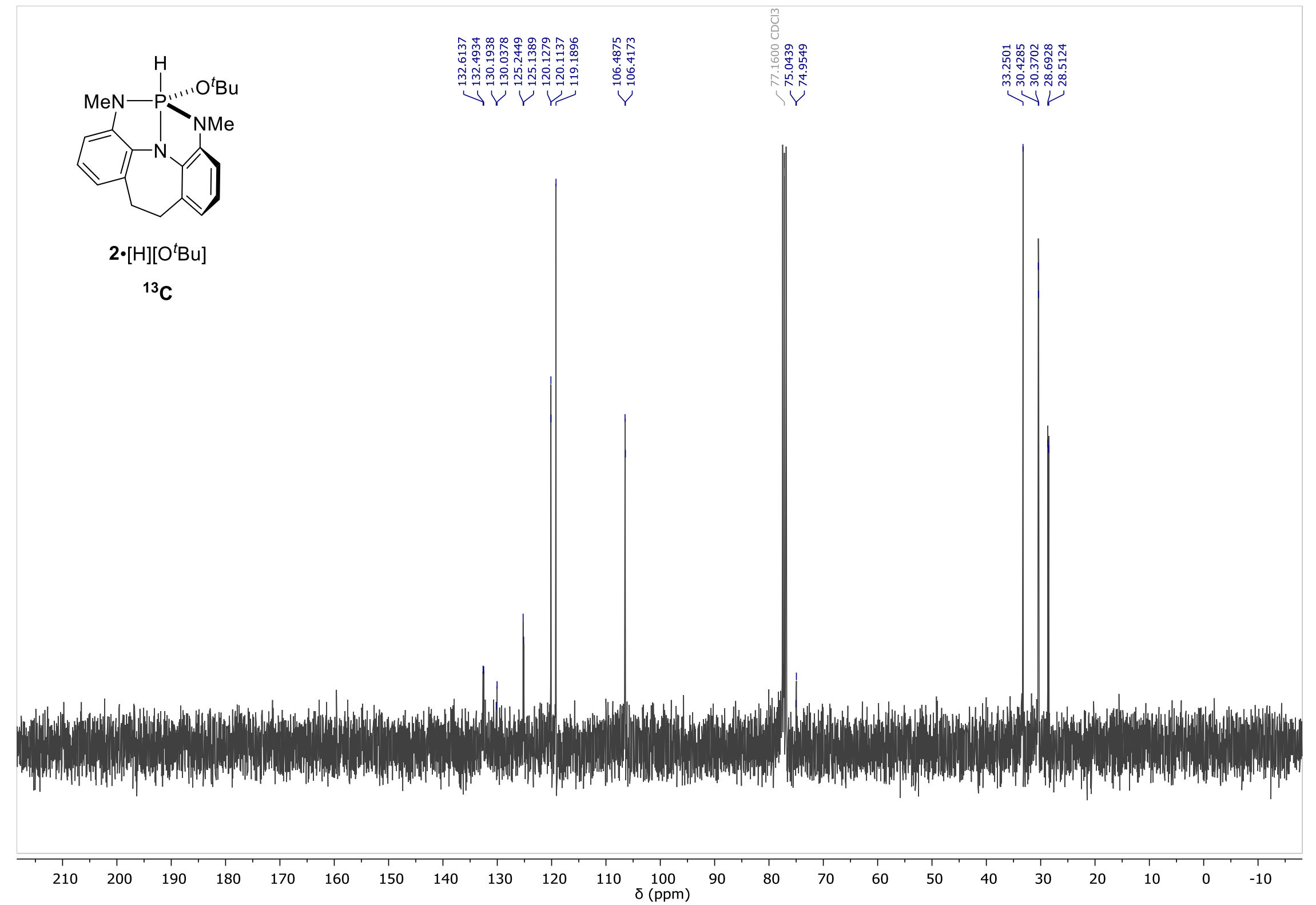

Figure S28. ${ }^{13} \mathrm{C}$ NMR spectrum of $\mathbf{2} \cdot[\mathrm{H}][\mathrm{O}-t-\mathrm{Bu}]$ recorded in $\mathrm{CDCl}_{3}$ at $23{ }^{\circ} \mathrm{C}$. 


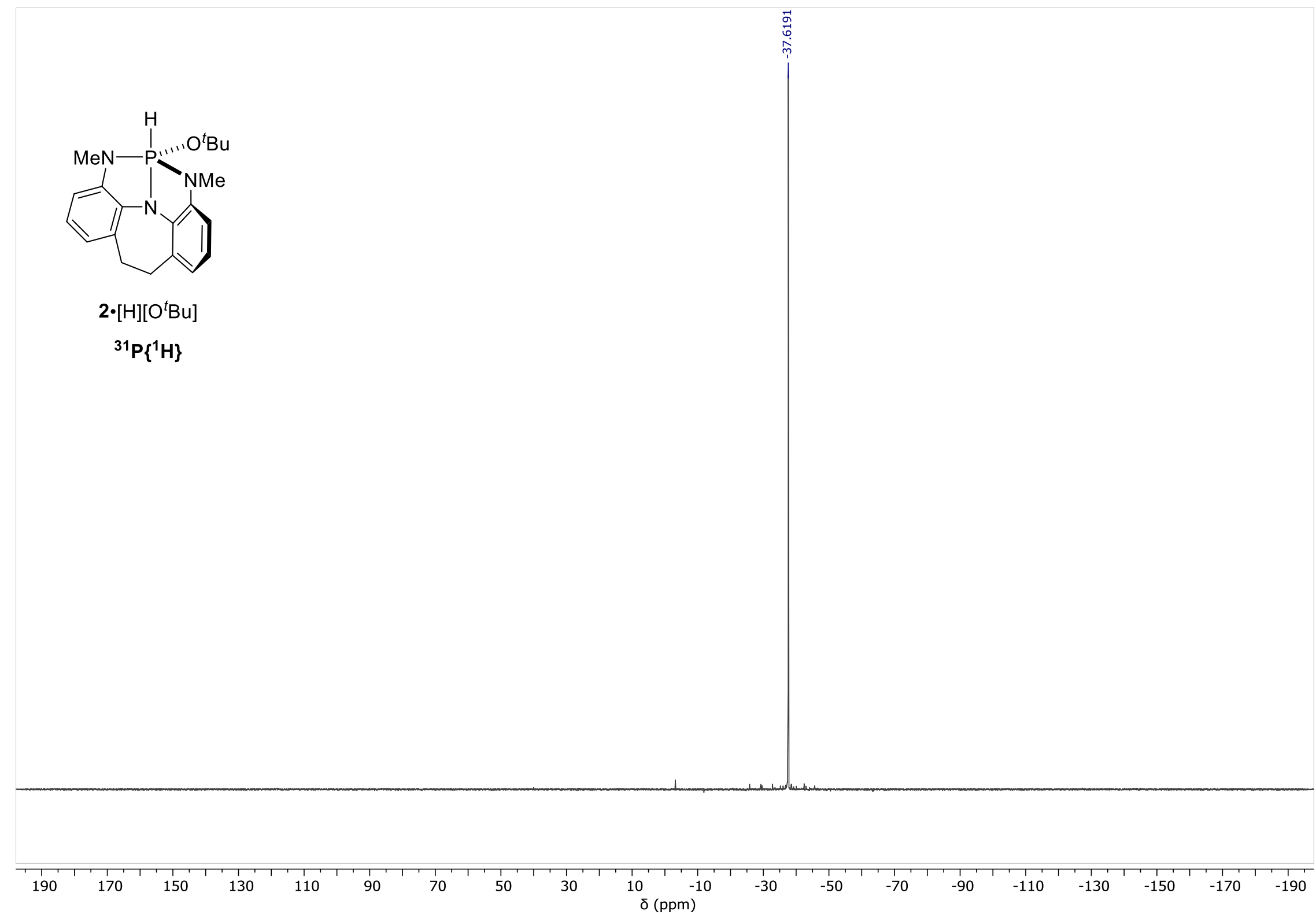

Figure S29. ${ }^{31} \mathrm{P}\left\{{ }^{1} \mathrm{H}\right\}$ NMR spectrum of $\mathbf{2} \cdot[\mathrm{H}][\mathrm{O}-\mathrm{t}$-Bu $]$ recorded in $\mathrm{CDCl}_{3}$ at $23{ }^{\circ} \mathrm{C}$. 


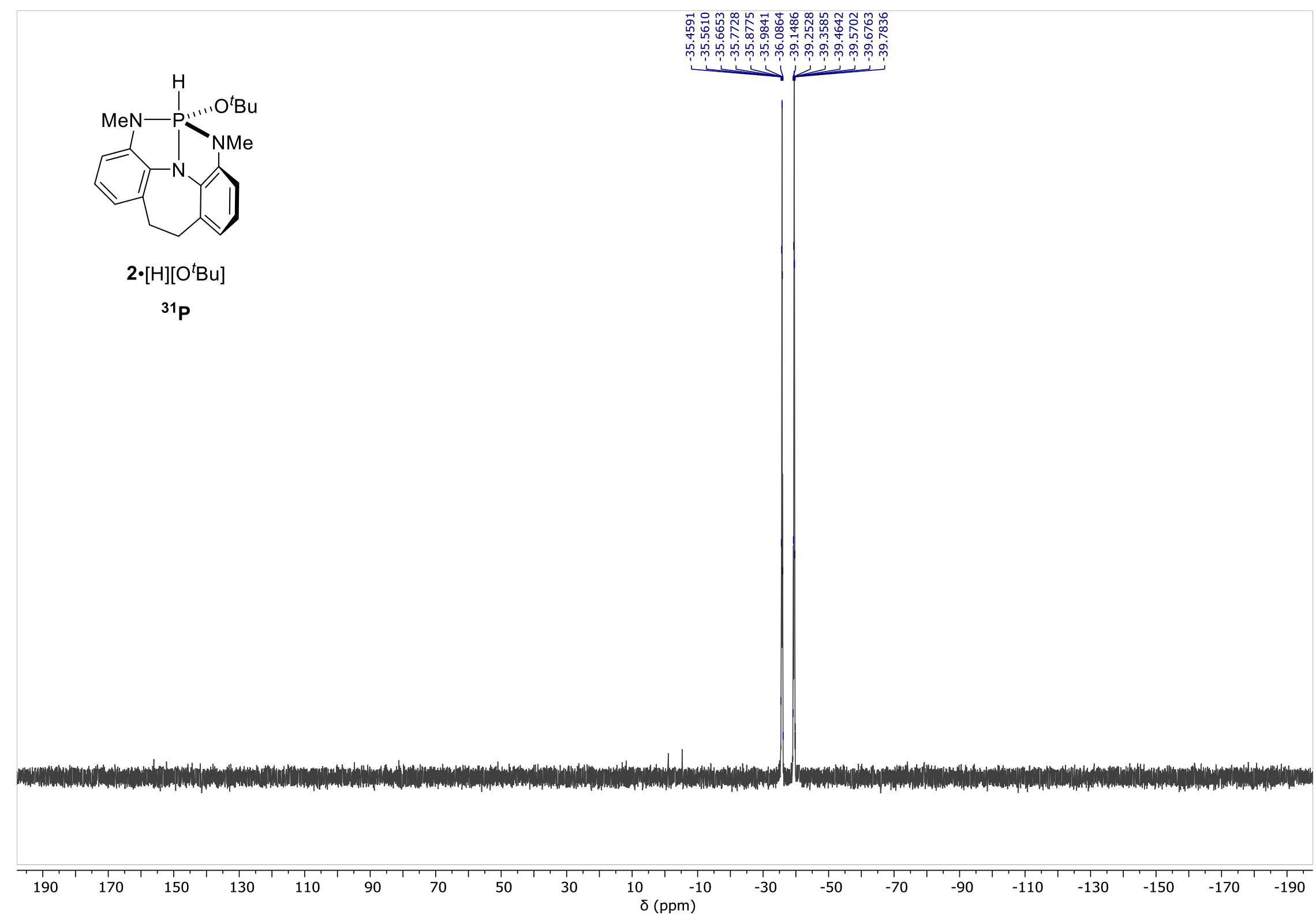

Figure S30. ${ }^{31} \mathrm{P}$ NMR spectrum of $2 \cdot[\mathrm{H}][\mathrm{O}-t-\mathrm{Bu}]$ recorded in $\mathrm{CDCl}_{3}$ at $23{ }^{\circ} \mathrm{C}$. 

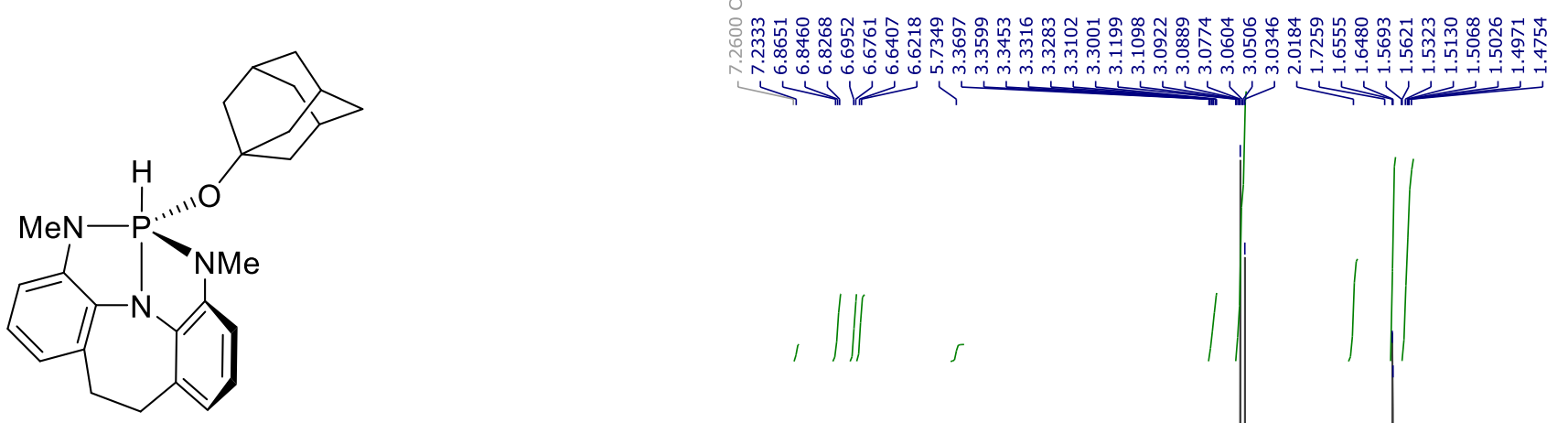

$2 \cdot[\mathrm{H}][\mathrm{O}-1-\mathrm{Ad}]$

${ }^{1} \mathrm{H}$
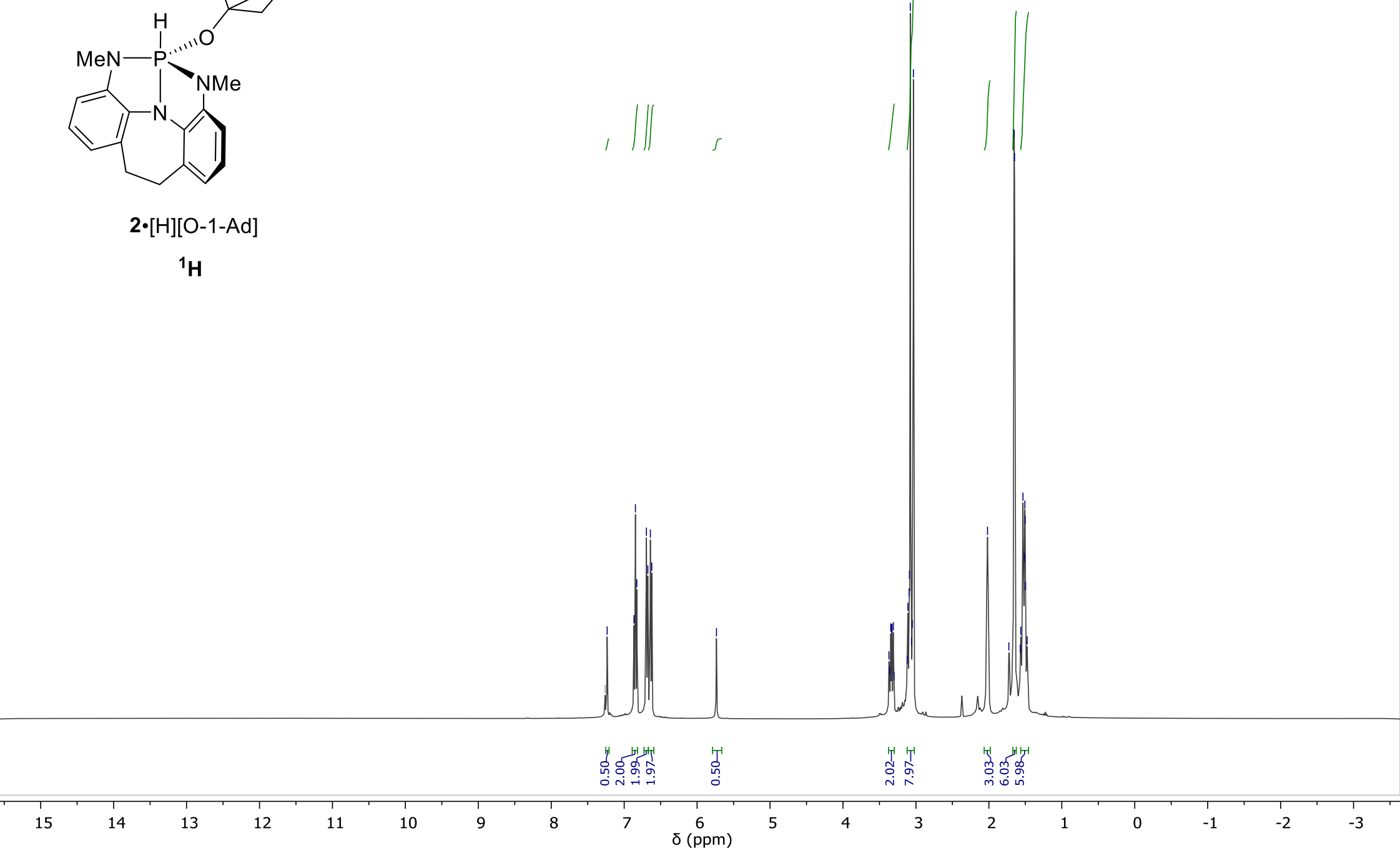

Figure S31. ${ }^{1} \mathrm{H}$ NMR spectrum of $\mathbf{2} \cdot[\mathrm{H}][\mathrm{O}-1-\mathrm{Ad}]$ recorded in $\mathrm{CDCl}_{3}$ at $23{ }^{\circ} \mathrm{C}$. 


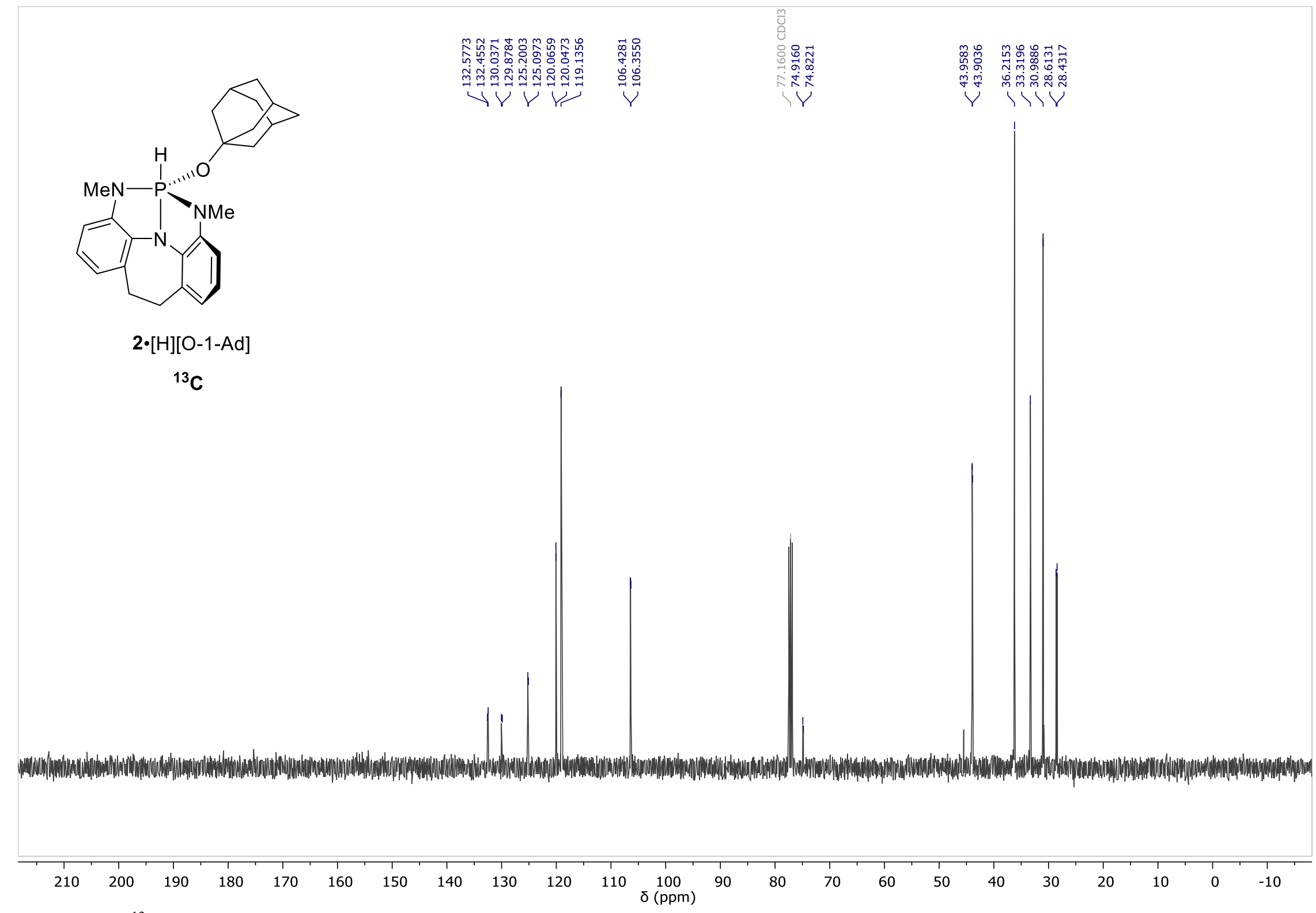

Figure S32. ${ }^{13} \mathrm{C}$ NMR spectrum of $\mathbf{2} \cdot[\mathrm{H}][\mathrm{O}-1-\mathrm{Ad}]$ recorded in $\mathrm{CDCl}_{3}$ at $23{ }^{\circ} \mathrm{C}$. 


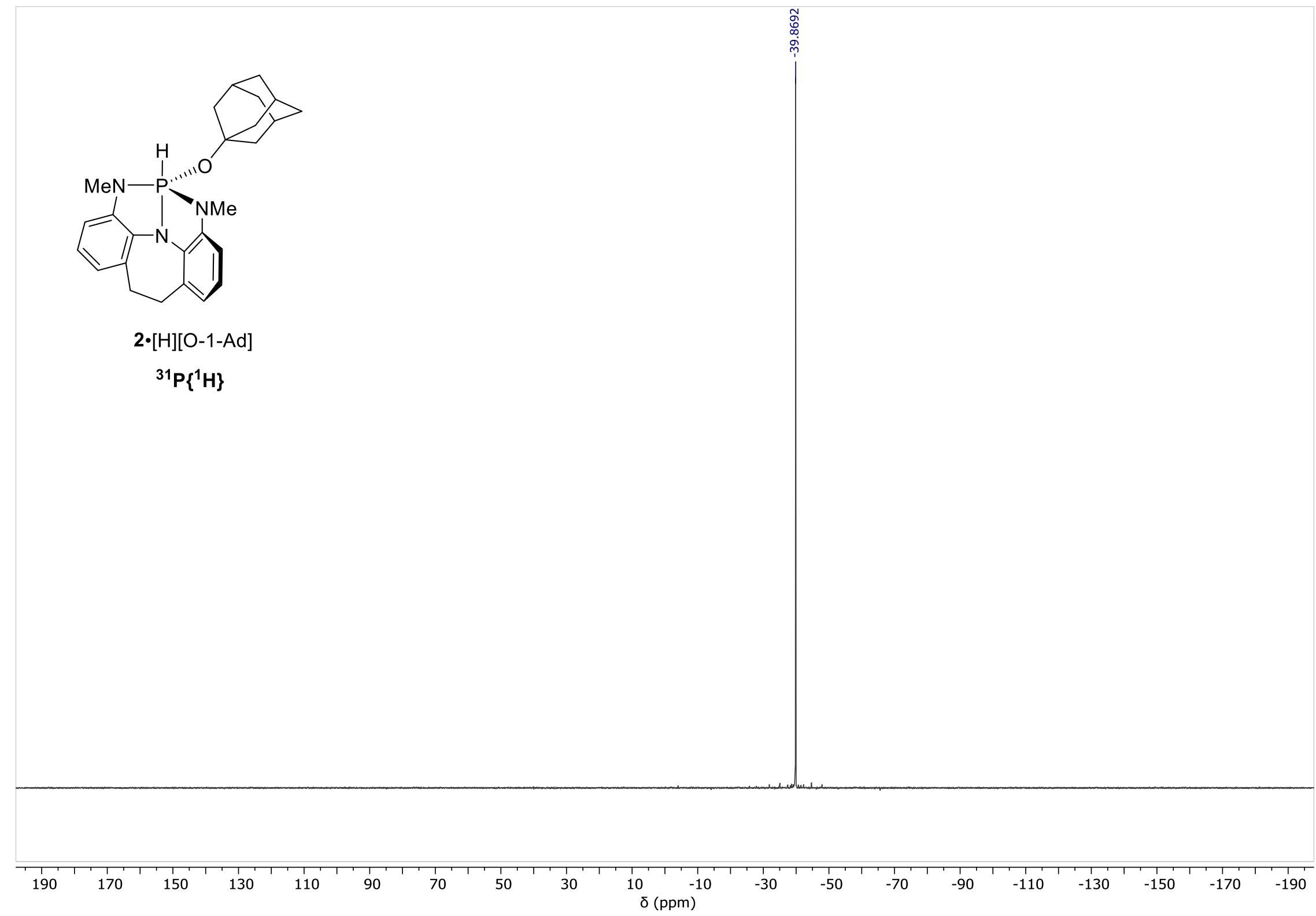

Figure S33. ${ }^{31} \mathrm{P}\left\{{ }^{1} \mathrm{H}\right\}$ NMR spectrum of $\mathbf{2} \cdot[\mathrm{H}][\mathrm{O}-1-\mathrm{Ad}]$ recorded in $\mathrm{CDCl}_{3}$ at $23{ }^{\circ} \mathrm{C}$. 


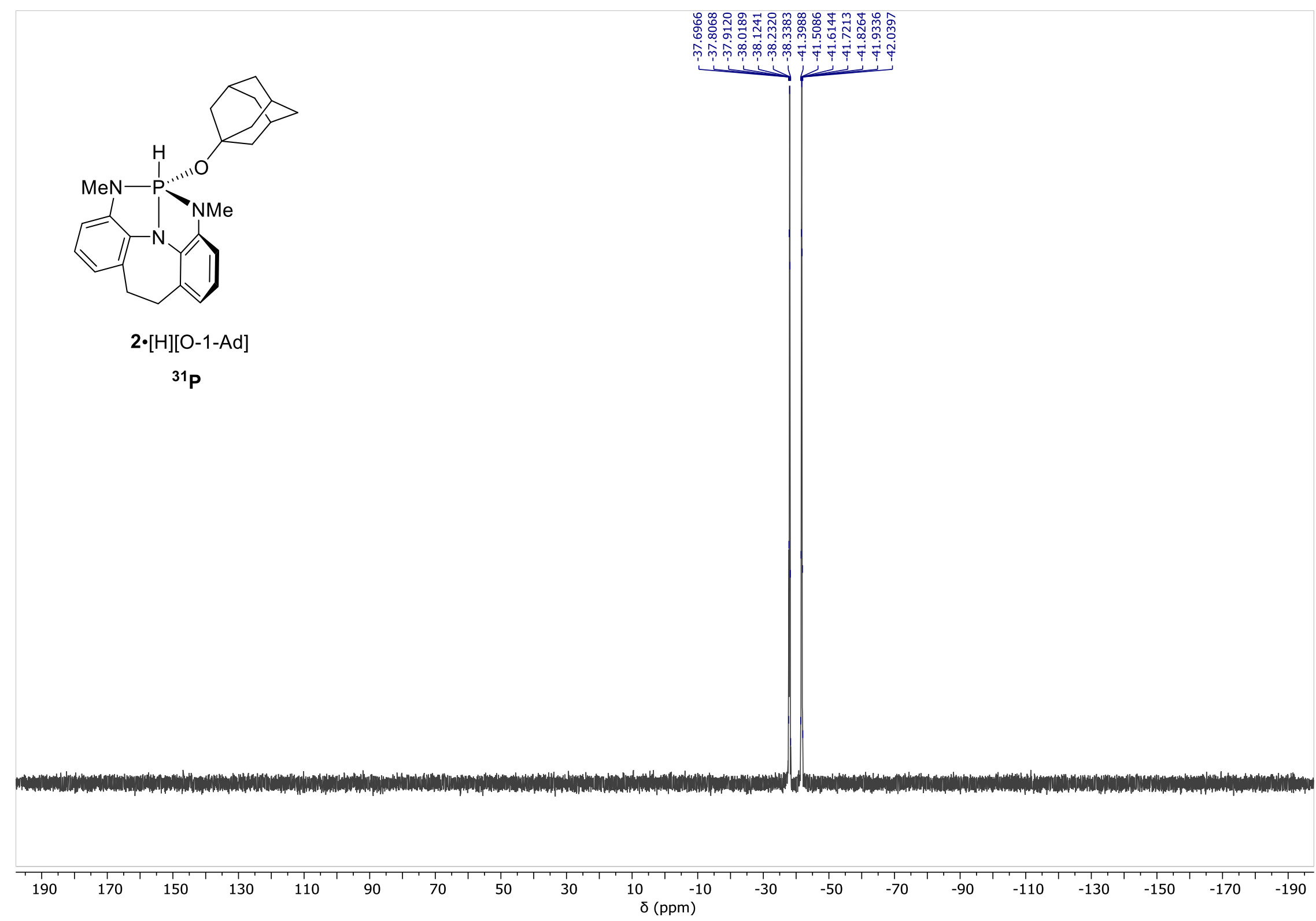

Figure S34. ${ }^{31} \mathrm{P}$ NMR spectrum of $2 \cdot[\mathrm{H}][\mathrm{O}-1-\mathrm{Ad}]$ recorded in $\mathrm{CDCl}_{3}$ at $23{ }^{\circ} \mathrm{C}$. 


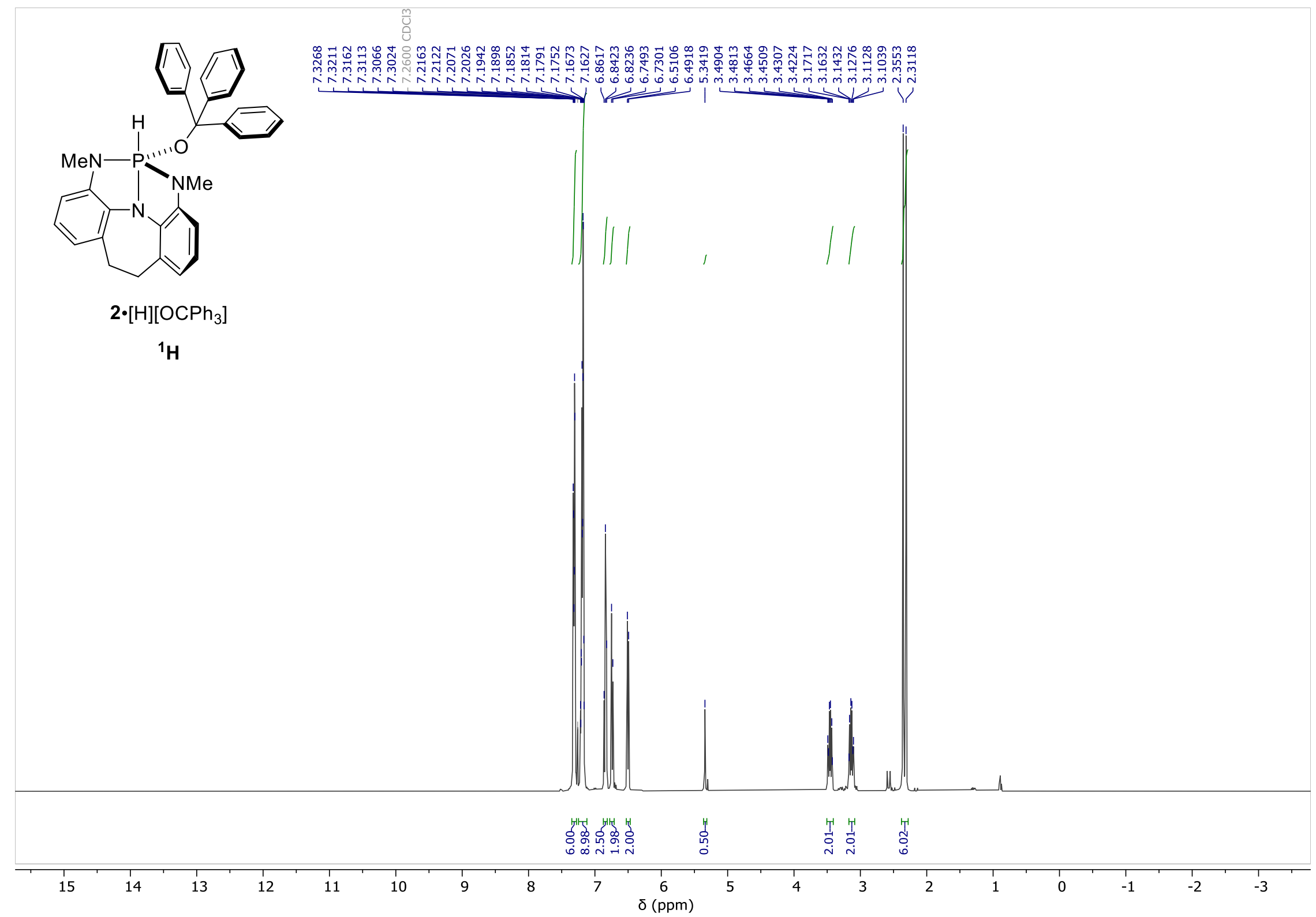

Figure S35. ${ }^{1} \mathrm{H}$ NMR spectrum of $\mathbf{2} \cdot[\mathrm{H}]\left[\mathrm{OCPh}_{3}\right]$ recorded in $\mathrm{CDCl}_{3}$ at $23{ }^{\circ} \mathrm{C}$. 


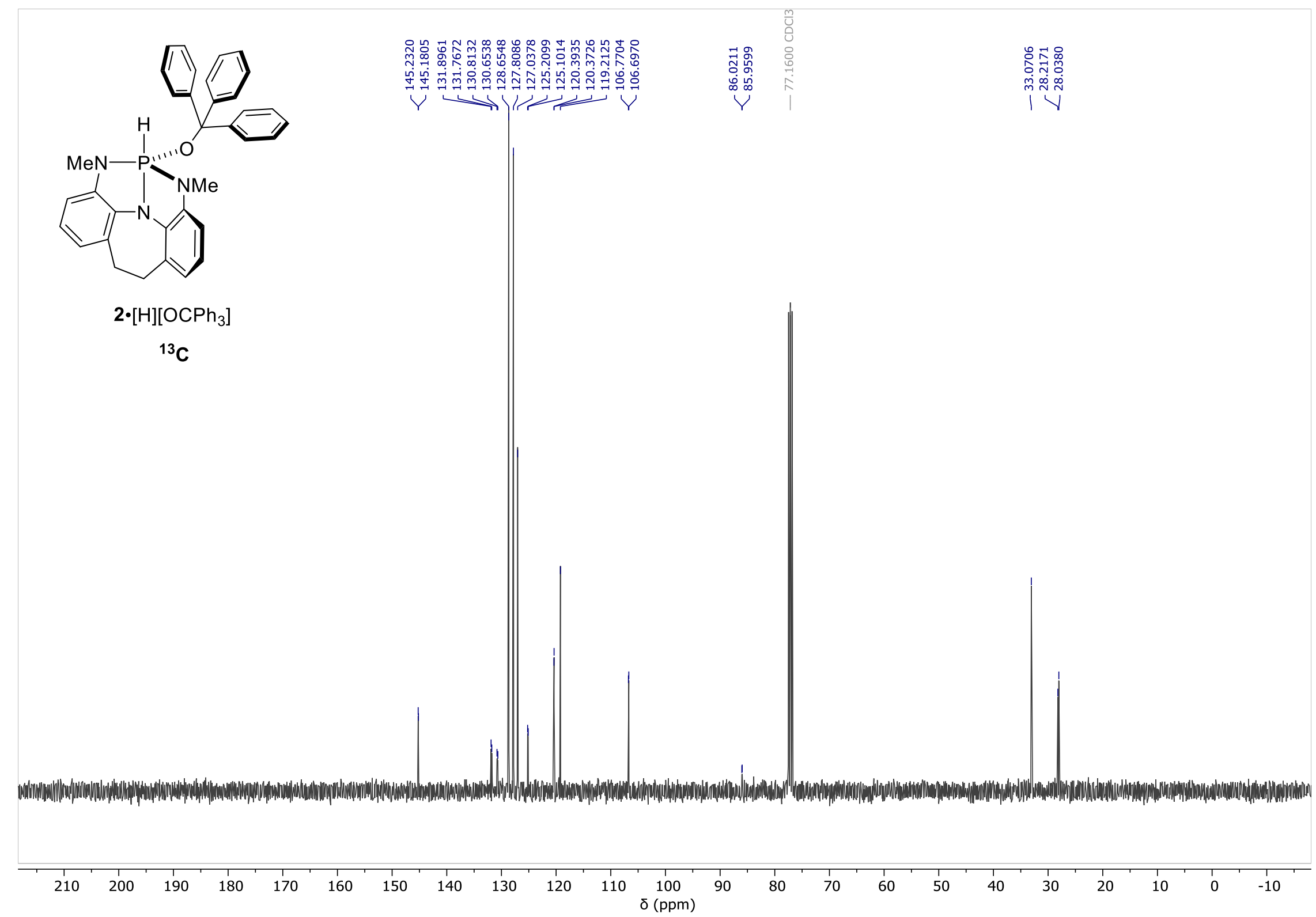

Figure S36. ${ }^{13} \mathrm{C}$ NMR spectrum of $2 \cdot[\mathrm{H}]\left[\mathrm{OCPh}_{3}\right]$ recorded in $\mathrm{CDCl}_{3}$ at $23{ }^{\circ} \mathrm{C}$. 

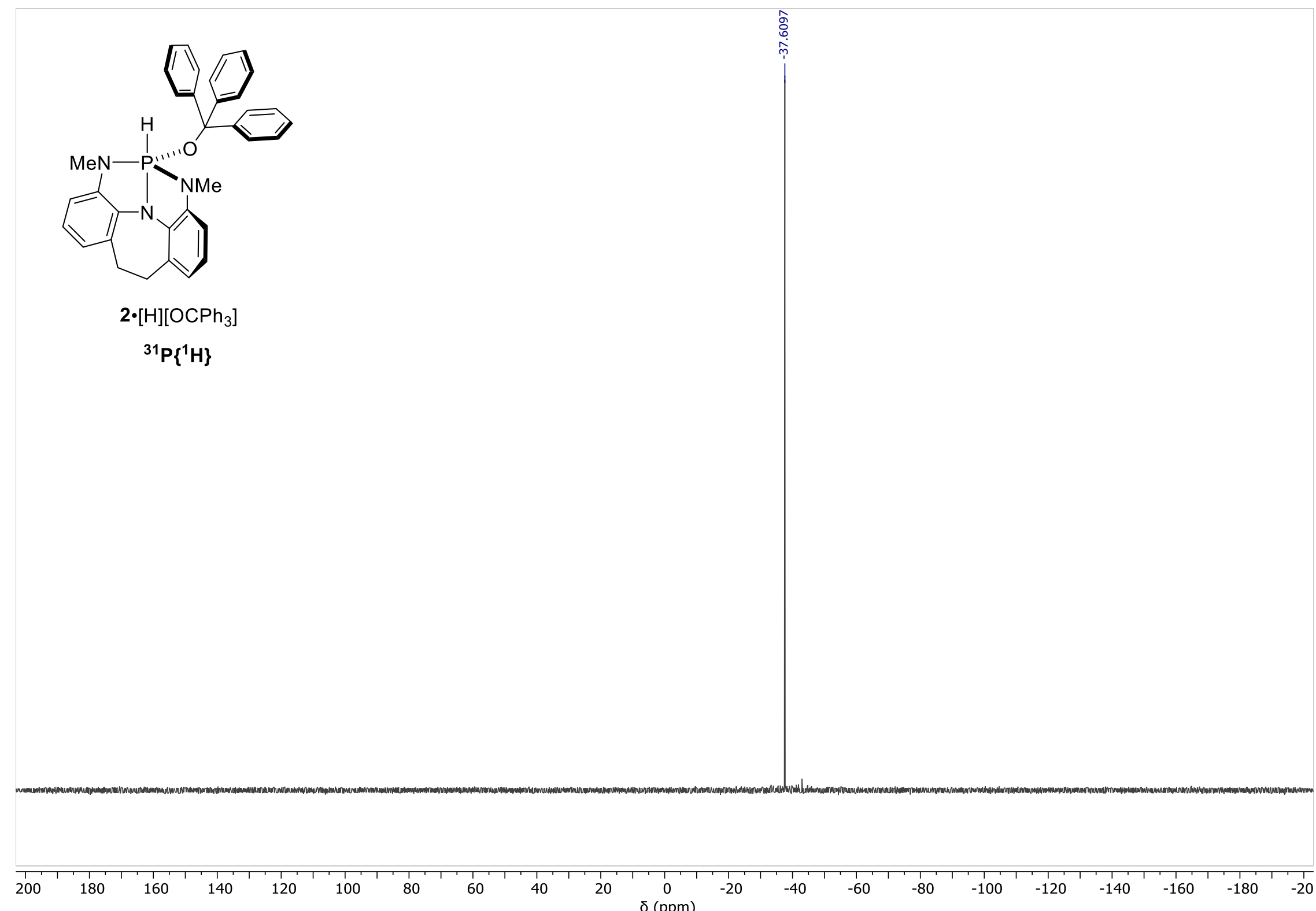

Figure S37. ${ }^{31} \mathrm{P}\left\{{ }^{1} \mathrm{H}\right\}$ NMR spectrum of $\mathbf{2} \cdot[\mathrm{H}]\left[\mathrm{OCPh}_{3}\right]$ recorded in $\mathrm{CDCl}_{3}$ at $23{ }^{\circ} \mathrm{C}$. 


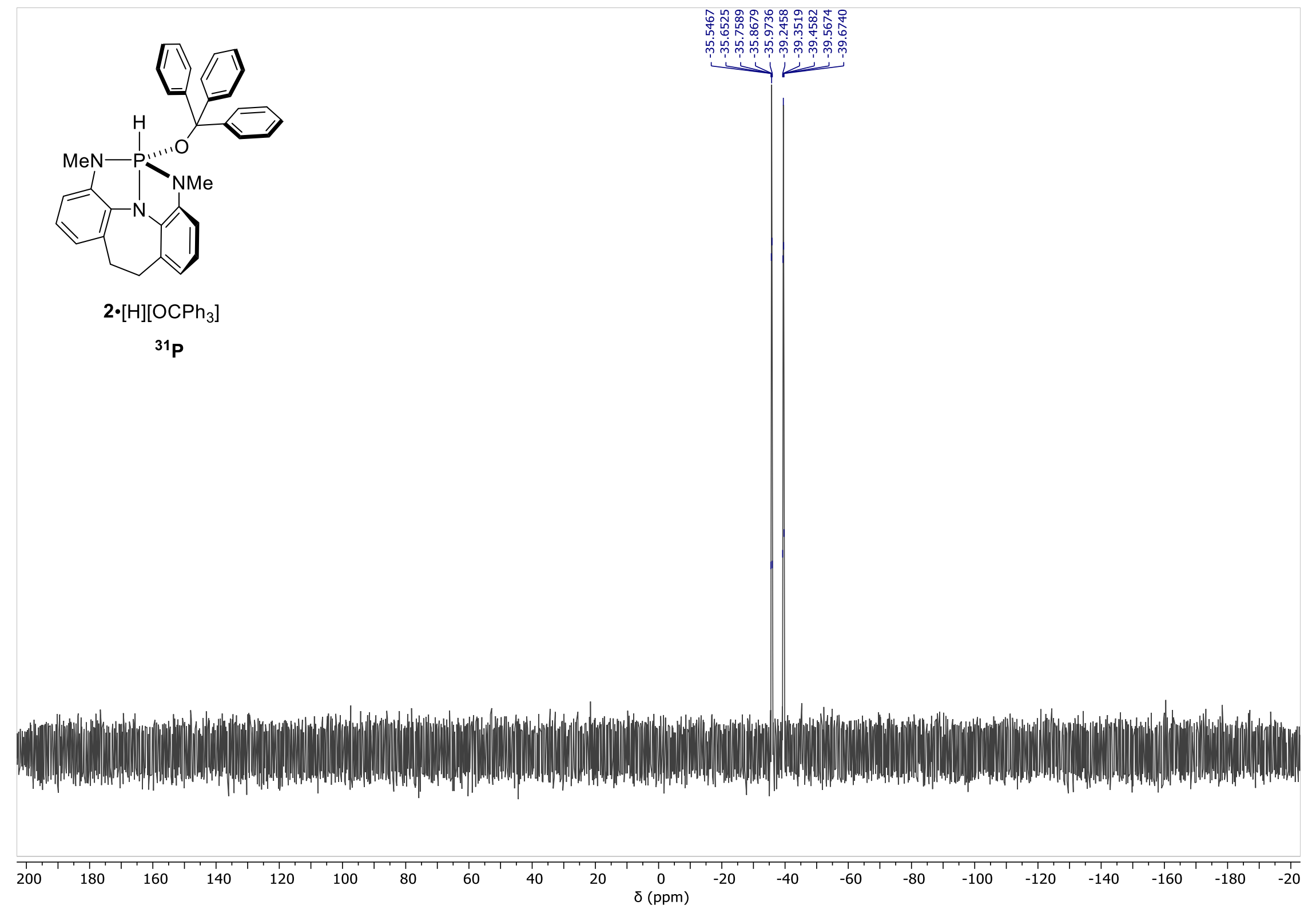

Figure S38. ${ }^{31} \mathrm{P}$ NMR spectrum of $\mathbf{2} \cdot[\mathrm{H}]\left[\mathrm{OCPh}_{3}\right]$ recorded in $\mathrm{CDCl}_{3}$ at $23{ }^{\circ} \mathrm{C}$. 


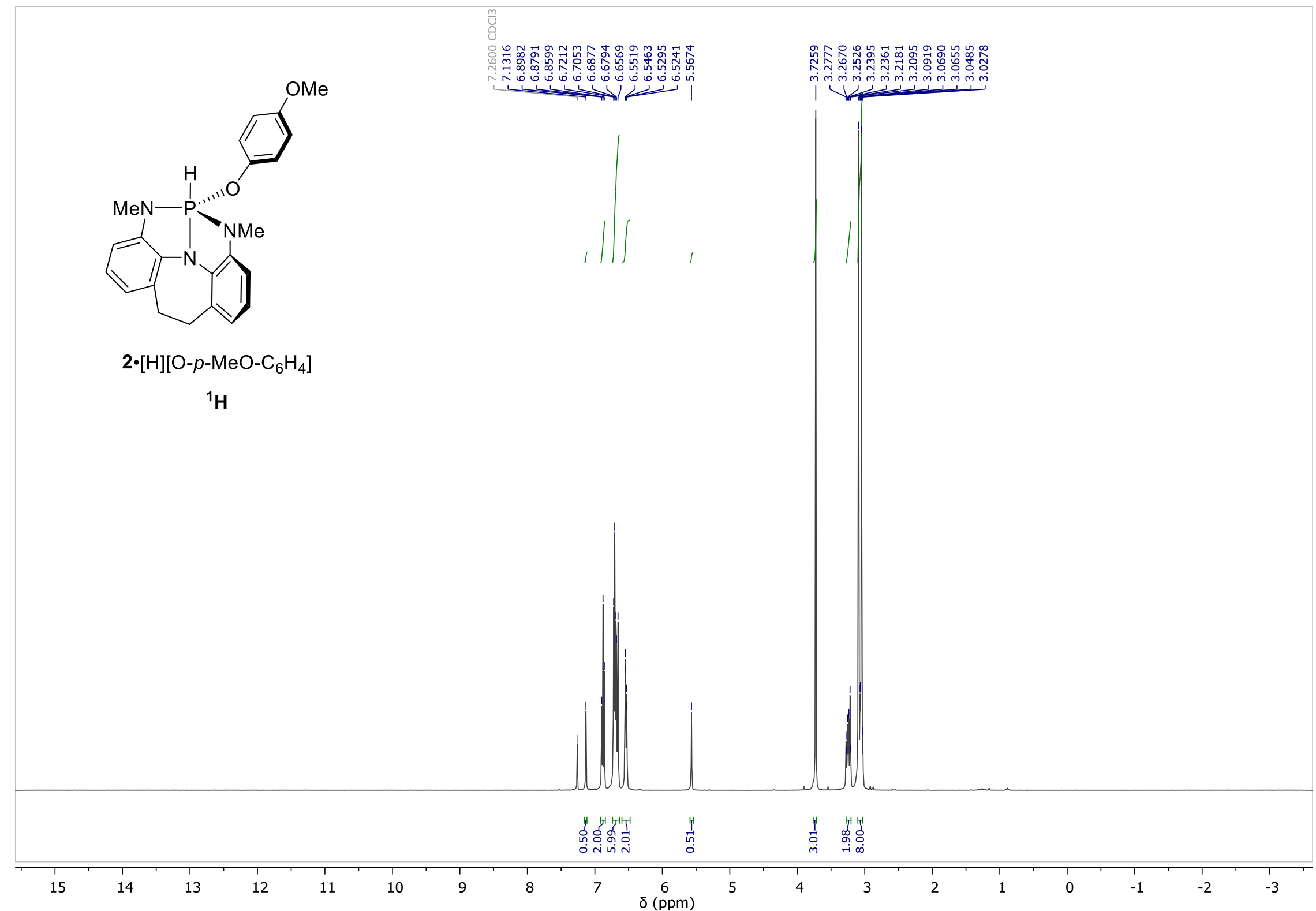

Figure S39. ${ }^{1} \mathrm{H}$ NMR spectrum of $\mathbf{2} \cdot[\mathrm{H}]\left[\mathrm{O}-p-\mathrm{MeO}-\mathrm{C}_{6} \mathrm{H}_{4}\right]$ recorded in $\mathrm{CDCl}_{3}$ at $23{ }^{\circ} \mathrm{C}$. 


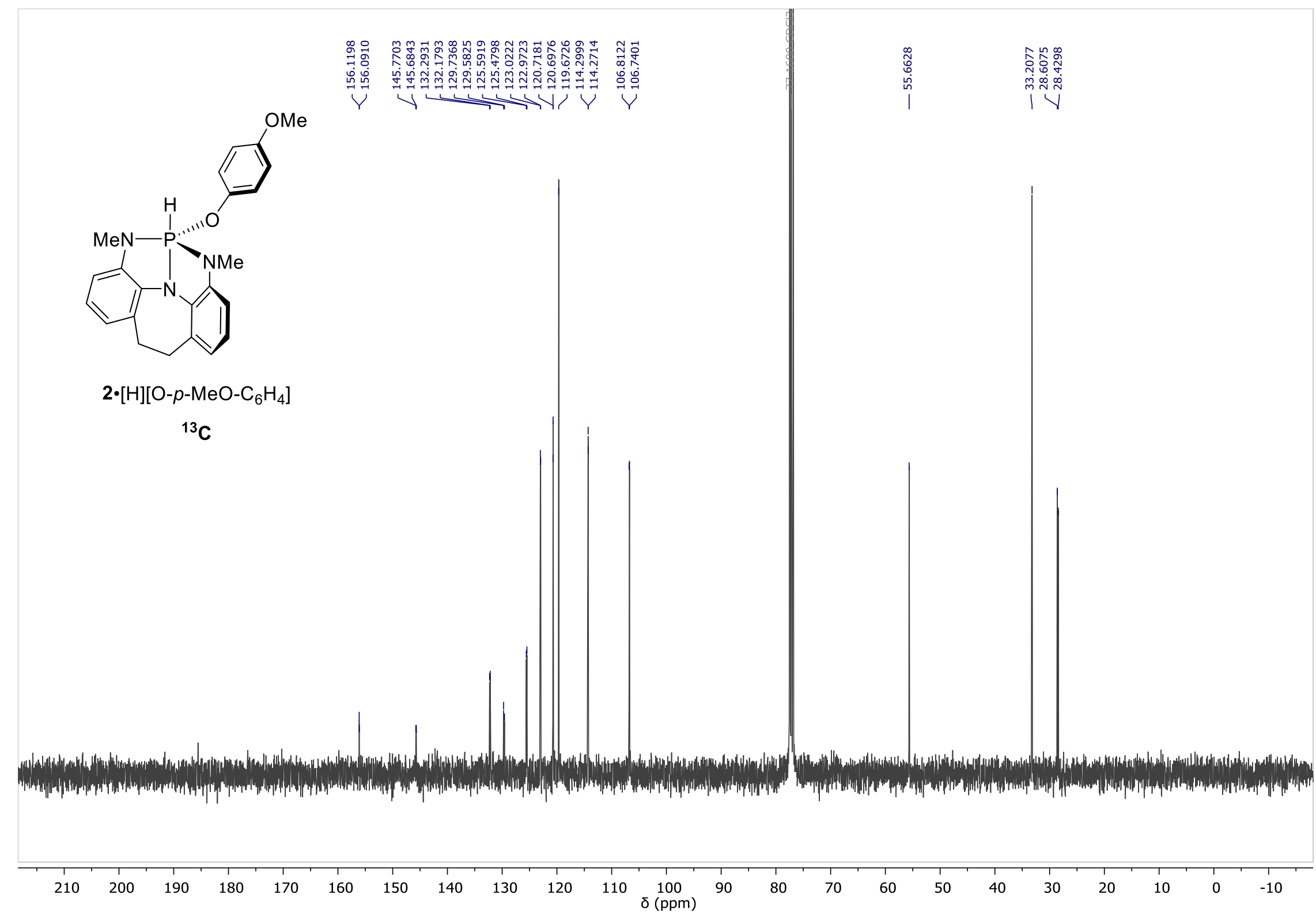

Figure S40. ${ }^{13} \mathrm{C}$ NMR spectrum of $\mathbf{2} \cdot[\mathrm{H}]\left[\mathrm{O}-p-\mathrm{MeO}-\mathrm{C}_{6} \mathrm{H}_{4}\right]$ recorded in $\mathrm{CDCl}_{3}$ at $23{ }^{\circ} \mathrm{C}$. 


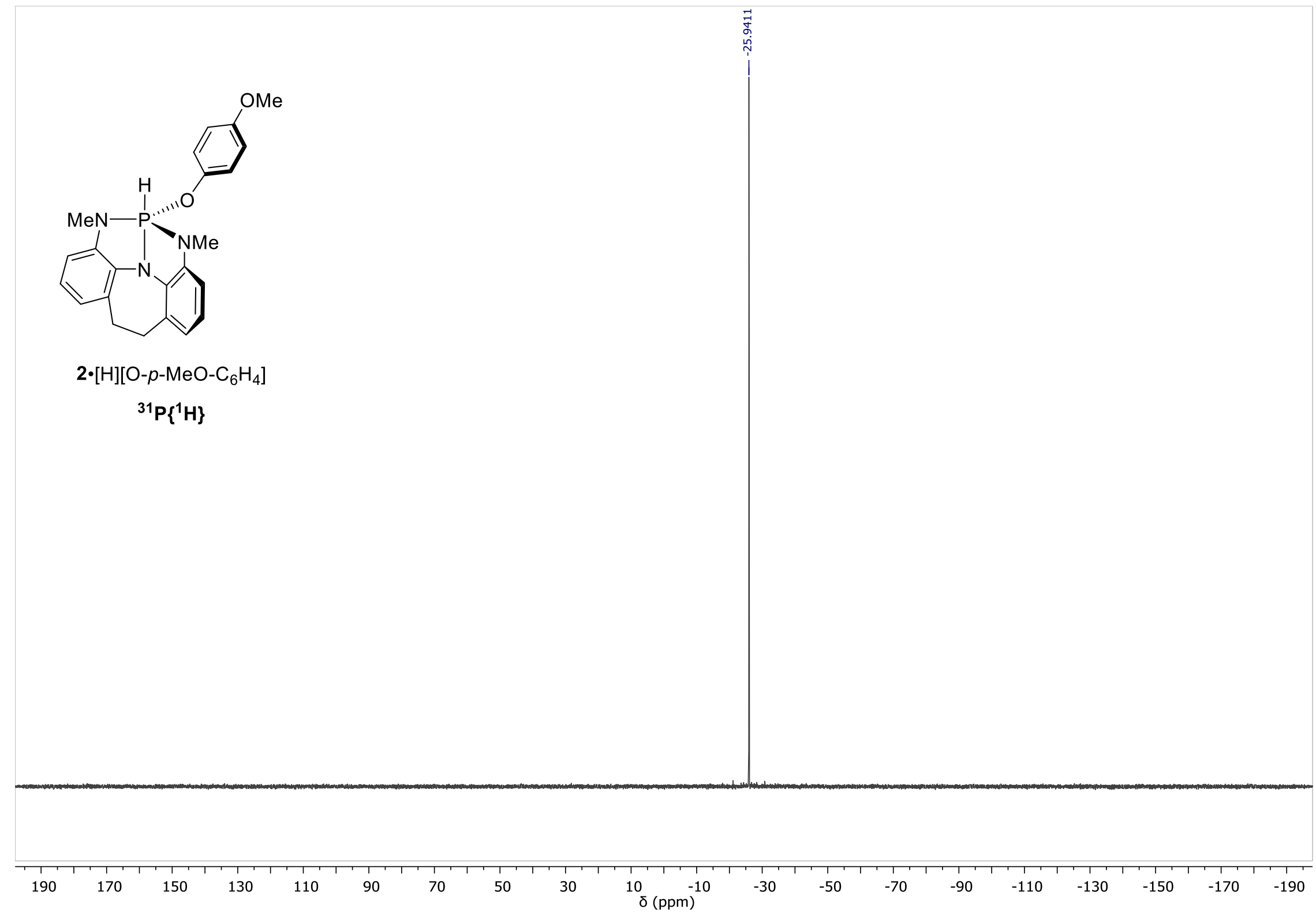

Figure S41. ${ }^{31} \mathrm{P}\left\{{ }^{1} \mathrm{H}\right\}$ NMR spectrum of $\mathbf{2} \cdot[\mathrm{H}]\left[\mathrm{O}-p-\mathrm{MeO}-\mathrm{C}_{6} \mathrm{H}_{4}\right]$ recorded in $\mathrm{CDCl}_{3}$ at $23{ }^{\circ} \mathrm{C}$. 


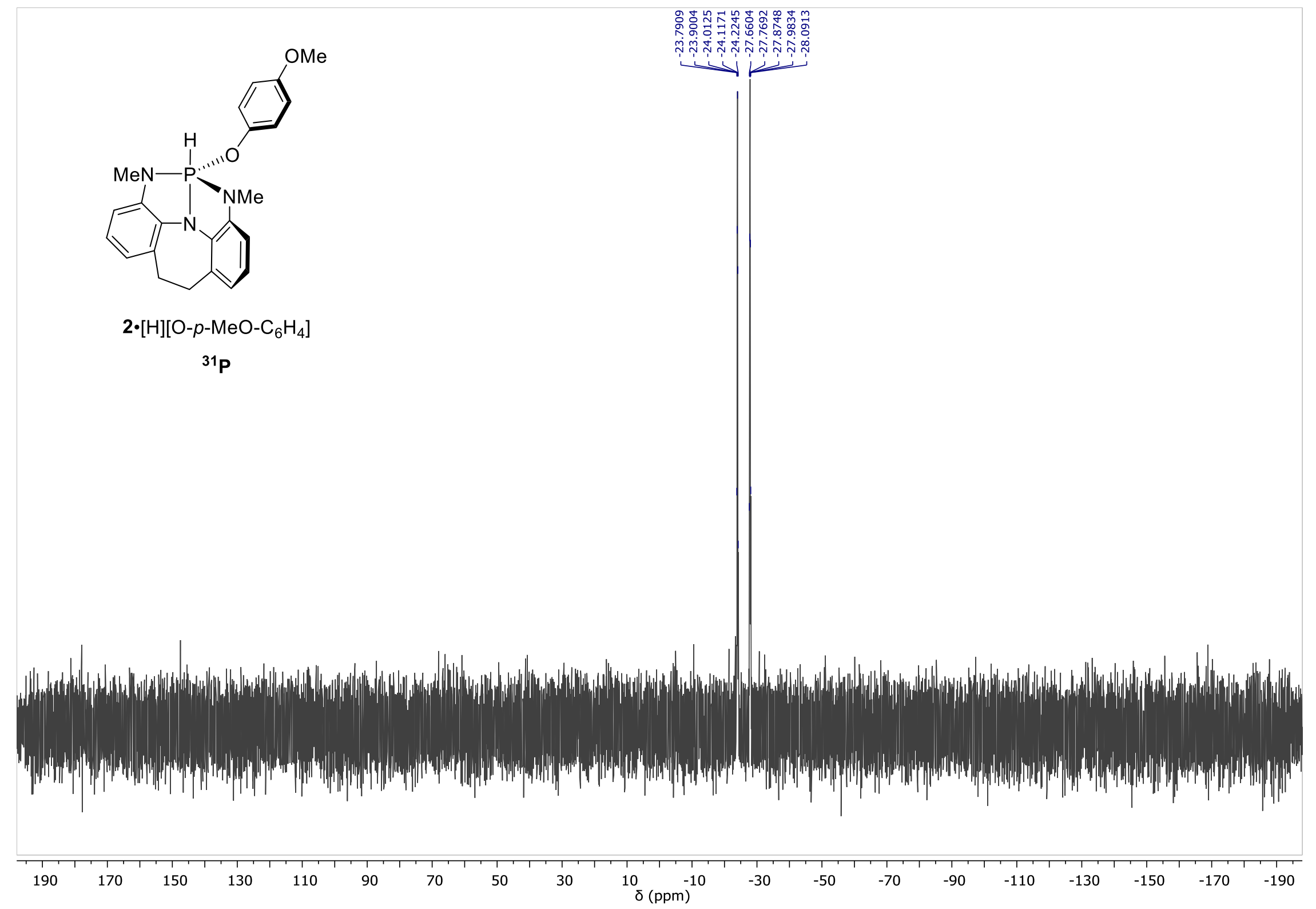

Figure S42. ${ }^{31} \mathrm{P}$ NMR spectrum of $\mathbf{2} \cdot[\mathrm{H}]\left[\mathrm{O}-p-\mathrm{MeO}-\mathrm{C}_{6} \mathrm{H}_{4}\right]$ recorded in $\mathrm{CDCl}_{3}$ at $23{ }^{\circ} \mathrm{C}$. 


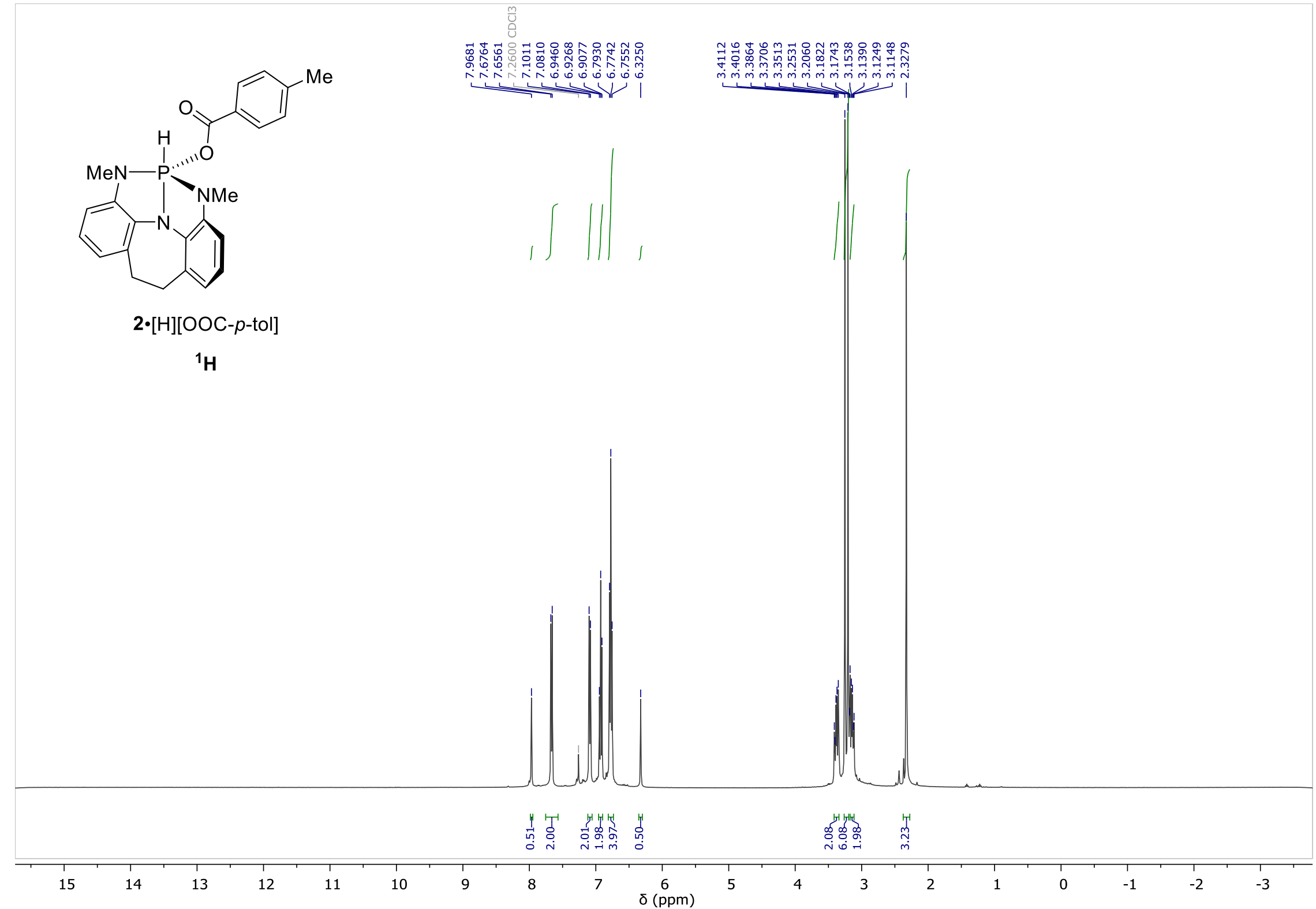

Figure S43. ${ }^{1} \mathrm{H}$ NMR spectrum of $\mathbf{2} \cdot[\mathrm{H}][\mathrm{OOC}-p$-tol $]$ recorded in $\mathrm{CDCl}_{3}$ at $23{ }^{\circ} \mathrm{C}$. 


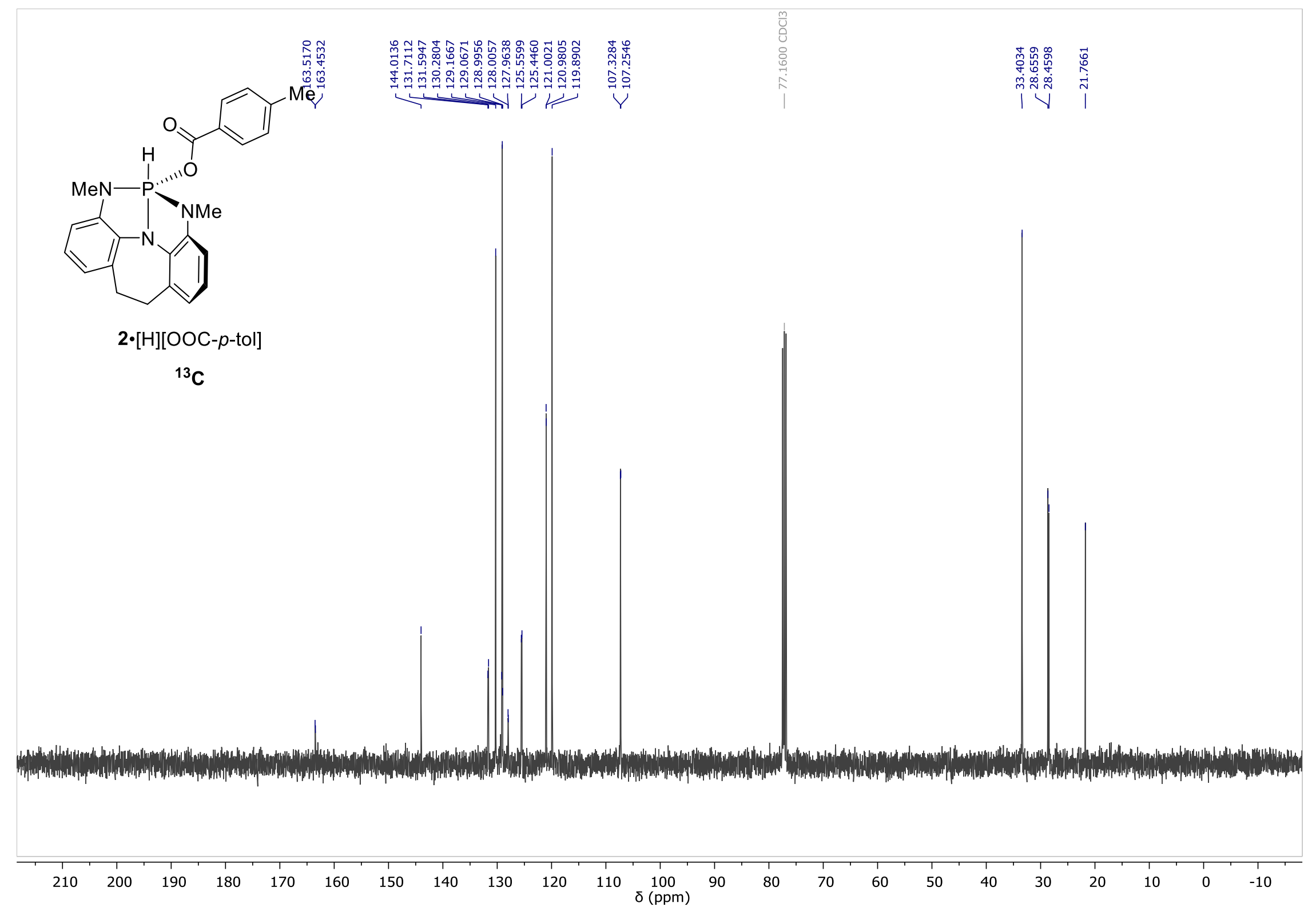

Figure S44. ${ }^{13} \mathrm{C}$ NMR spectrum of $\mathbf{2} \cdot[\mathrm{H}]\left[\mathrm{OOC}-p\right.$-tol] recorded in $\mathrm{CDCl}_{3}$ at $23{ }^{\circ} \mathrm{C}$. 


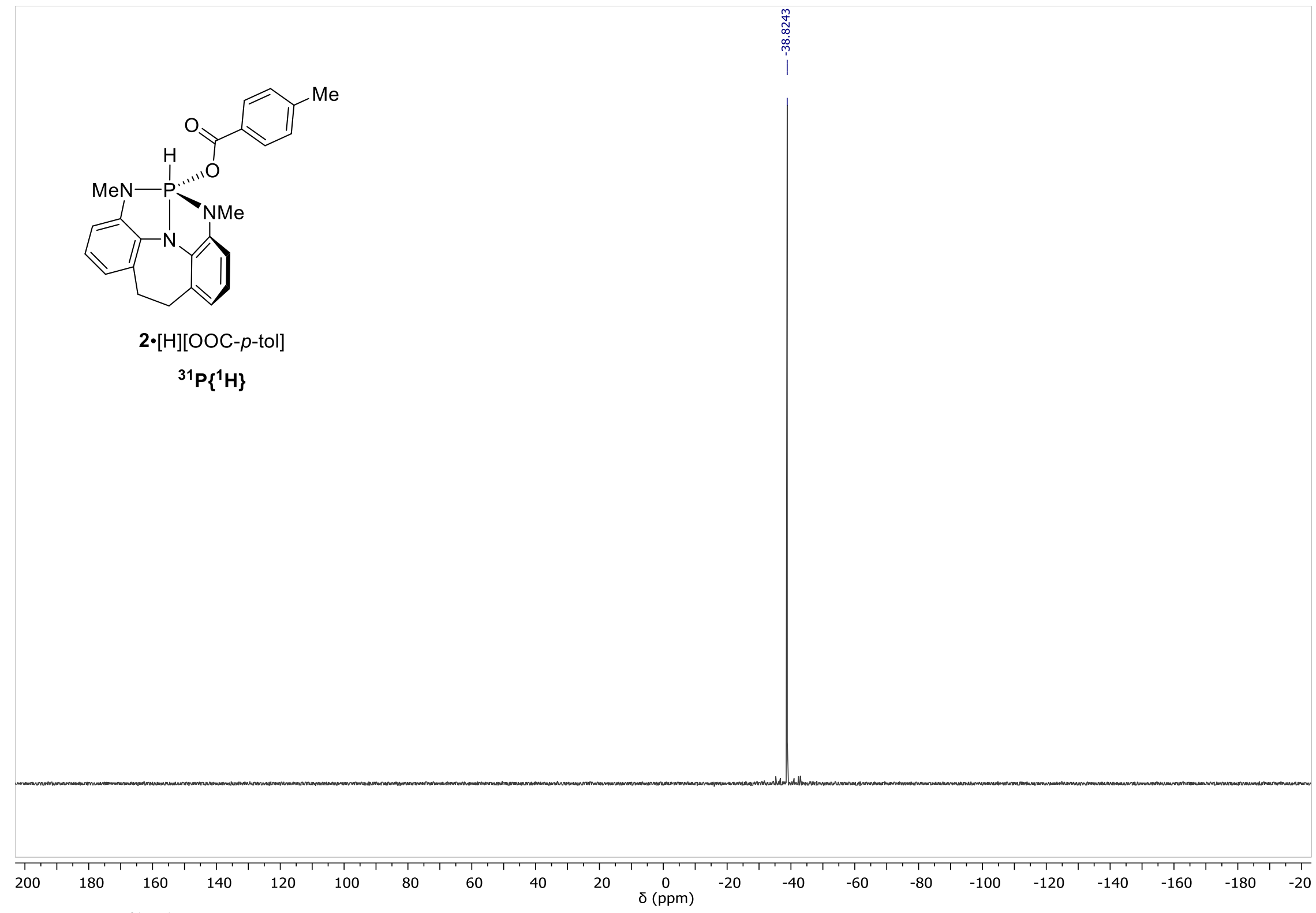

Figure S45. ${ }^{31} \mathrm{P}\left\{{ }^{1} \mathrm{H}\right\}$ NMR spectrum of $\mathbf{2} \cdot[\mathrm{H}][\mathrm{OOC}-p$-tol $]$ recorded in $\mathrm{CDCl}_{3}$ at $23{ }^{\circ} \mathrm{C}$. 


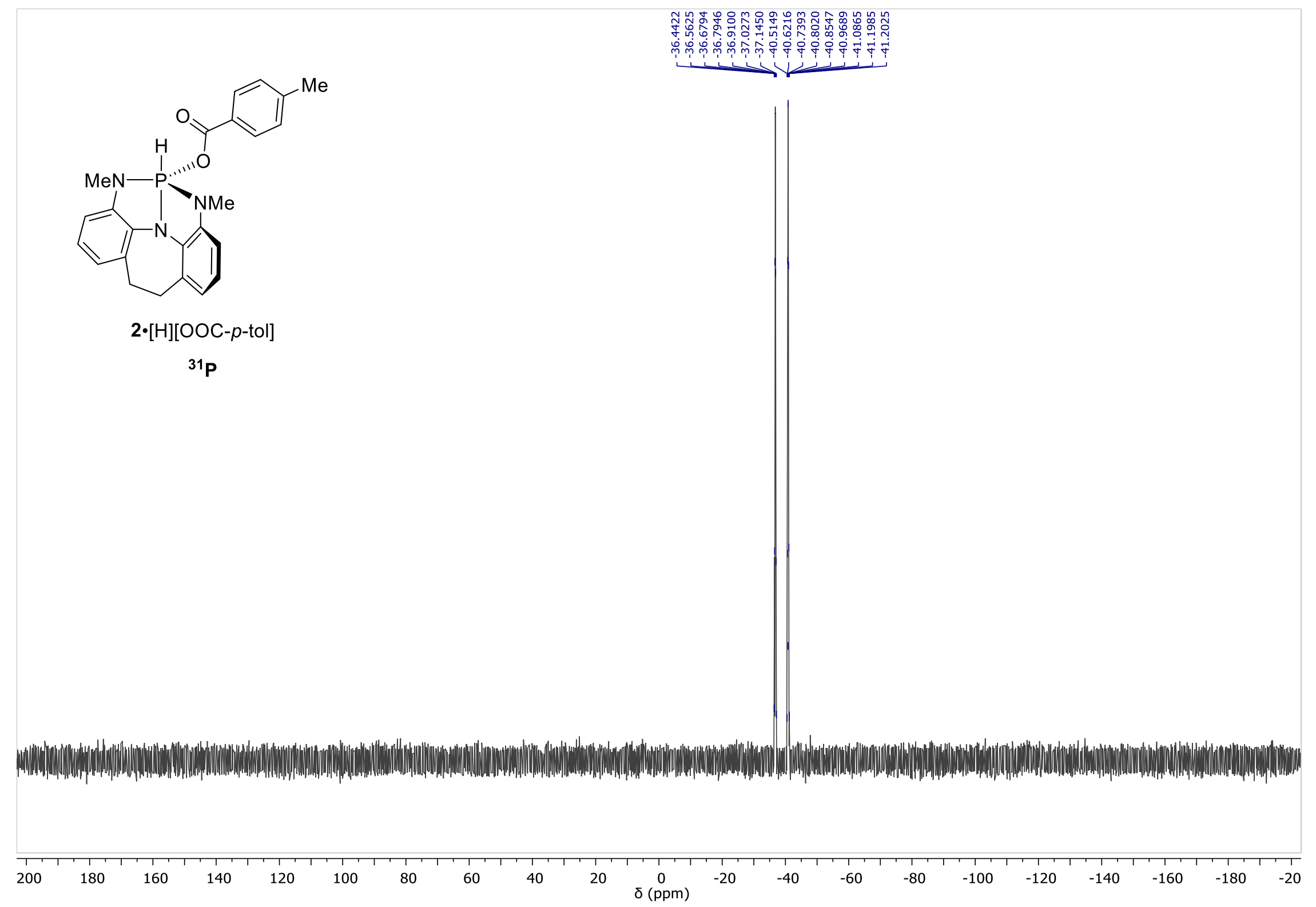

Figure S46. ${ }^{31} \mathrm{P}$ NMR spectrum of $\mathbf{2} \cdot[\mathrm{H}][\mathrm{OOC}$ - $p$-tol $]$ recorded in $\mathrm{CDCl}_{3}$ at $23{ }^{\circ} \mathrm{C}$. 


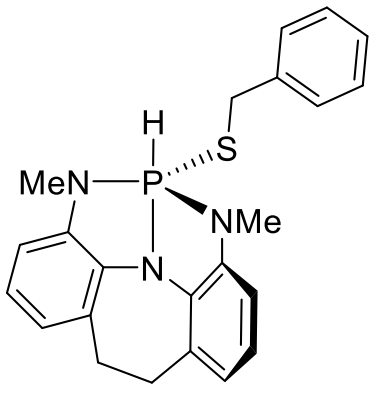

2•[H][SBn]

${ }^{1} \mathrm{H}$

\section{.}
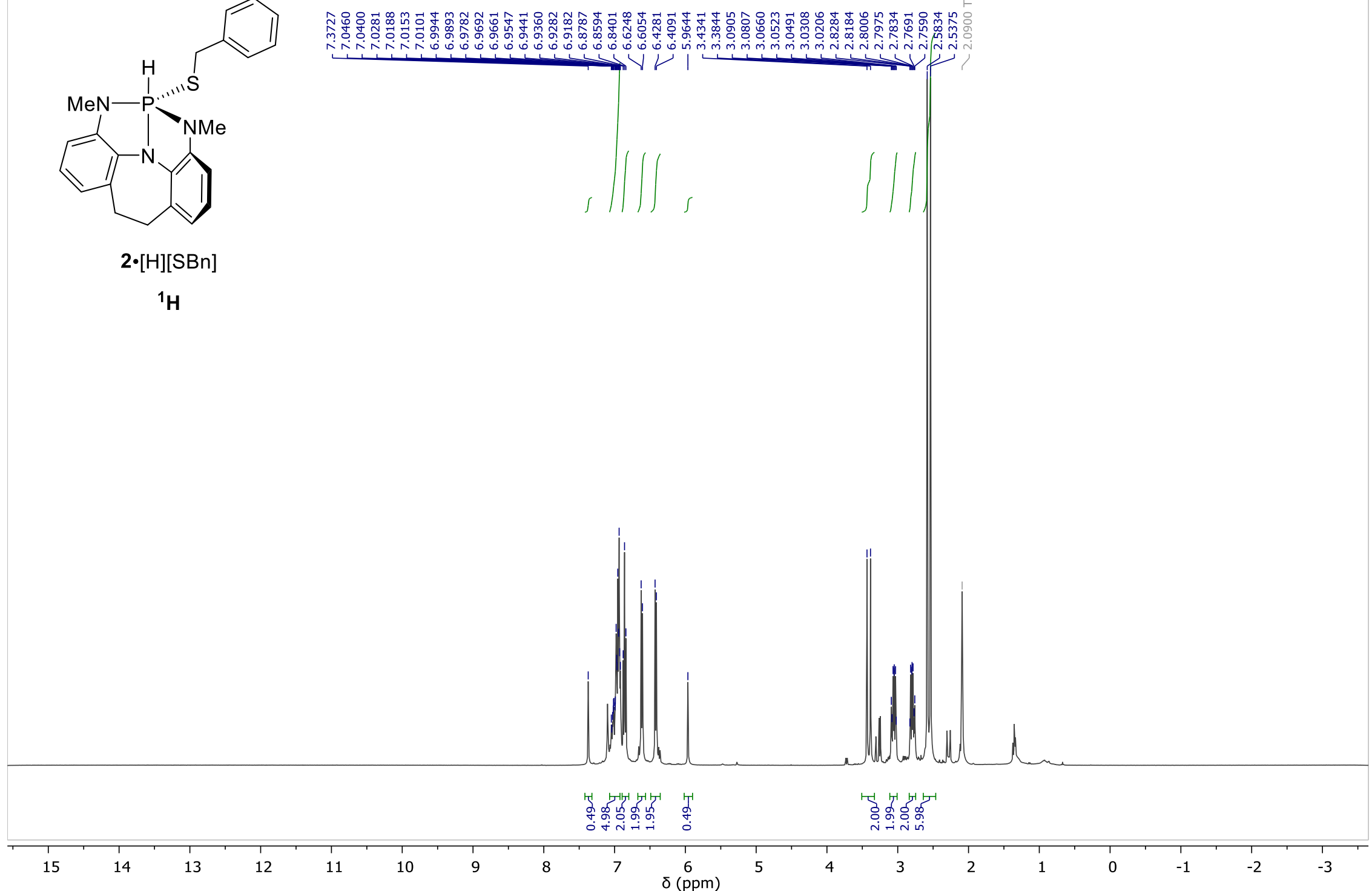

Figure S47. ${ }^{1} \mathrm{H}$ NMR spectrum of $2 \cdot[\mathrm{H}][\mathrm{SBn}]$ recorded in toluene- $d_{8}$ at $23{ }^{\circ} \mathrm{C}$. 


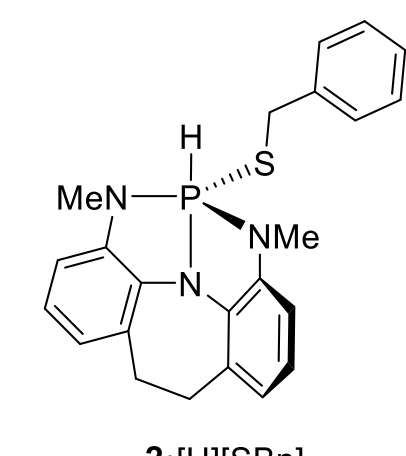

$2 \cdot[\mathrm{H}][\mathrm{SBn}]$

${ }^{13} \mathrm{C}$

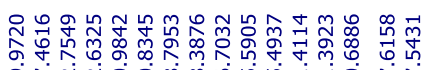

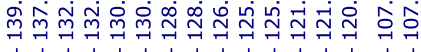

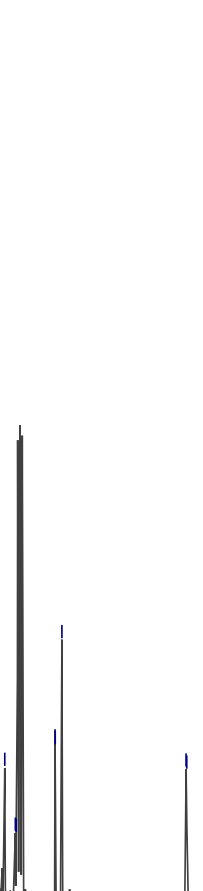

210

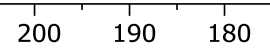

170

160

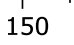

130

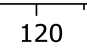

$110 \quad 100$

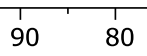

70

60

50

Figure S48. ${ }^{13} \mathrm{C}$ NMR spectrum of $2 \cdot[\mathrm{H}][\mathrm{SBn}]$ recorded in toluene- $d_{8}$ at $23{ }^{\circ} \mathrm{C}$. 


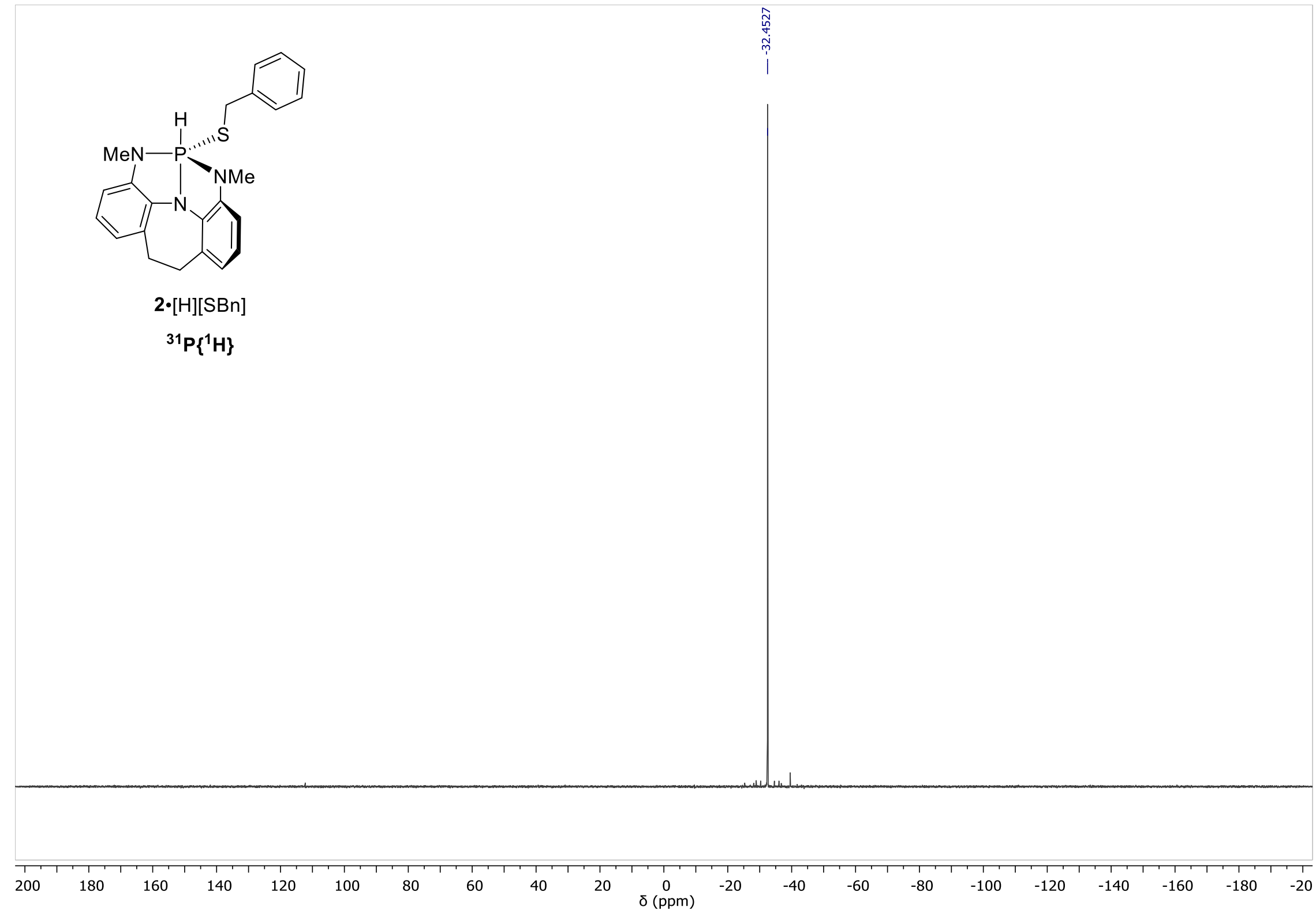

Figure S49. ${ }^{31} \mathrm{P}\left\{{ }^{1} \mathrm{H}\right\}$ NMR spectrum of $2 \cdot[\mathrm{H}][\mathrm{SBn}]$ recorded in toluene- $d_{8}$ at $23{ }^{\circ} \mathrm{C}$. 


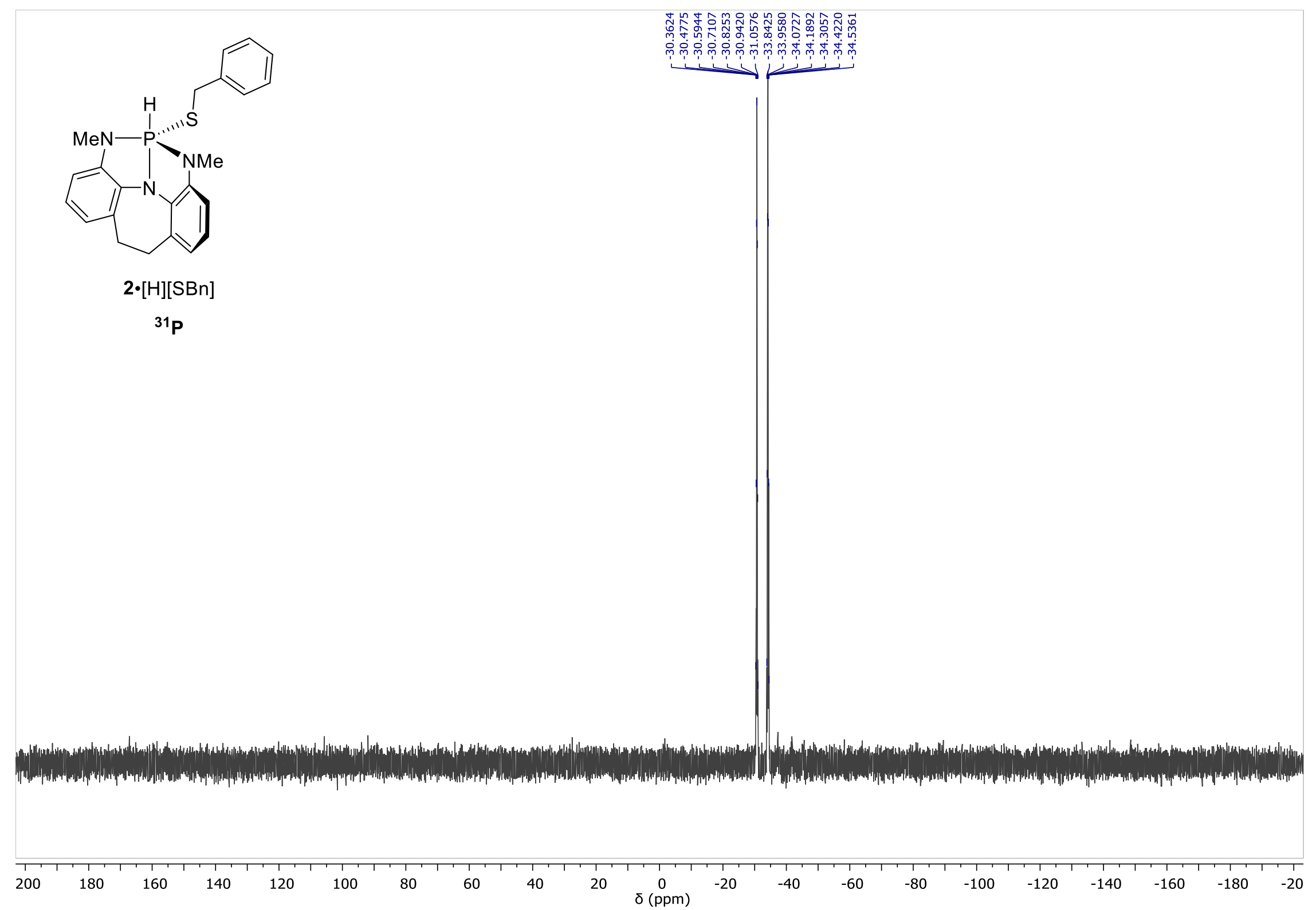

Figure S50. ${ }^{31} \mathrm{P}$ NMR spectrum of $2 \cdot[\mathrm{H}][\mathrm{SBn}]$ recorded in toluene- $d_{8}$ at $23{ }^{\circ} \mathrm{C}$. 


\section{References}

${ }^{1}$ Zhao, W.; McCarthy, S. M.; Lai, T. Y.; Yennawar, H. P.; Radosevich, A. T. J. Am. Chem. Soc. 2014, 136, 17634-17644.

${ }^{2}$ Elliott, E.-C.; Regan, S. L.; Maggs, J. L.; Bowkett, E. R.; Parry, L. J.; Williams, D. P.; Park, B. K.; Stachulski, A. V. J. Med. Chem. 2012, 55, 9773-9784.

3 (a) Wang, D.; Lindeman, S. V.; Fiedler, A. T. Inorg. Chem. 2015, 54, 8744-8754. (b) Munha, R. F.; Zarkesh, R. A.; Heyduk, A. F. Inorg. Chem. 2013, 52, 11244-11255. (c) Murdock, K. C.; Lin, Y.-I.; Thomas, J. P.; Lang, S. A. Jr. J. Med. Chem. 1978, 21, 403-405.

${ }^{4}$ Reich, H. J. WinDNMR-Pro for Windows. Downloaded via the internet at: http://www.chem.wisc.edu/areas/reich/plt/windnmr.htm.

${ }^{5}$ Neese, F. WIREs Comput. Mol. Sci., 2012, 2, 73-78.

${ }^{6}$ Grimme, S.; Antony, J.; Ehrlich, S.; Krieg, H. J. Chem. Phys. 2010, 132, 154104.

7 (a) Krishnan, R.; Binkley, J. S.; Seeger, R.; Pople, J. A. J. Chem. Phys. 1980, 72, 650-654. (b) McLean, A. D.; Chandler, G. S. J. Chem. Phys. 1980, 72, 5639-5648.

${ }^{8}$ Becke, A. D. J. Chem. Phys. 1993, 98, 5648-5652. (b) Lee, C.; Yang, W.; Parr, R. G. Phys. Rev. B 1988, 37, 785-789.

9 (a) Weigend, F.; Ahlrichs, R. Phys. Chem. Chem. Phys. 2005, 7, 3297-3305. (b) Weigend, F. Phys. Chem. Chem. Phys. 2006, 8, $1057-1065$. 